\title{
Towards Oka-Cartan theory for algebras of holomorphic functions on coverings of Stein manifolds I
}

\author{
Alexander Brudnyi and Damir Kinzebulatov
}

\begin{abstract}
We develop complex function theory within certain algebras of holomorphic functions on coverings of Stein manifolds. This, in particular, includes the results on holomorphic extension from complex submanifolds, corona-type theorems, properties of divisors, holomorphic analogs of the Peter-Weyl approximation theorem, Hartogs-type theorems, characterization of uniqueness sets. The model examples of these algebras are:

(1) Bohr's algebra of holomorphic almost periodic functions on tube domains;

(2) algebra of all fibrewise bounded holomorphic functions (e.g., arising in the corona problem for $H^{\infty}$ ).

Our approach is based on an extension of the classical Oka-Cartan theory to coherent-type sheaves on the maximal ideal spaces of these algebras - topological spaces having some features of complex manifolds.
\end{abstract}

\section{Introduction}

In the 1930-50s, K. Oka and H. Cartan laid down the foundations of the modern function theory of several complex variables. In particular, they introduced the notion of a coherent sheaf and proved the following fundamental facts:

(A) Every germ of a coherent sheaf $\mathcal{A}$ on a Stein manifold $X$ is generated by its global sections ("Cartan theorem $A$ ").

(B) The sheaf cohomology groups $H^{i}(X, \mathcal{A})(i \geq 1)$ are trivial ("Cartan theorem $\left.B^{\prime \prime}\right)$.

Let us recall that a sheaf of modules over the sheaf of germs of holomorphic functions on $X$ is called coherent if locally both the sheaf and its sheaf of relations

Mathematics Subject Classification (2010): 32A38, 32K99.

Keywords: Oka-Cartan theory, algebras of holomorphic functions, coverings of complex manifolds. 
are finitely generated. The class of coherent sheaves is closed under natural operations. Most sheaves that arise in complex analysis are coherent, see, e.g., [20] for details.

A Stein manifold is a complex manifold that admits a proper holomorphic embedding into some $\mathbb{C}^{n}$.

Cartan theorems A and B together with their numerous corollaries constitute the so-called Oka-Cartan theory of Stein manifolds. Applying these theorems one obtains solutions (in algebra $\mathcal{O}(X)$ of holomorphic functions on a Stein manifold $X)$ to all classical problems of function theory of several complex variables (such as Cousin problems, the Poincaré problem, the Levi problem, the problem of holomorphic extension from complex analytic subsets, corona problems and many others, see, e.g., [20]).

Further development of complex function theory was motivated, in part, by the problems requiring to study properties of holomorphic functions satisfying special conditions (e.g., certain growth conditions 'at infinity'). As a result, the questions whether the problems of the classical complex function theory can be solved within a proper subclass of $\mathcal{O}(X)$, e.g., consisting of holomorphic $L^{p}$-functions on $X, 1 \leq p \leq \infty$, with respect to a suitable measure, started to play an important role (cf. the pioneering papers of L. Hörmander, J. J. Kohn, C. Morrey). However, trying to incorporate in the proofs such conditions as $L^{p}$-summability, the classical Oka-Cartan theory encounters considerable difficulties. In particular, one has to amplify the sheaf-theoretic methods of Oka-Cartan, e.g., by integral representation formulas on complex manifolds, estimates for solutions of $\bar{\partial}$-equations, etc. (see [25]).

Nevertheless, in some cases the methods of the Oka-Cartan theory can be extended to work within some special classes of holomorphic functions. The present paper studies one of these cases.

Definition 1.1. A holomorphic function $f$ defined on a regular covering $p: X \rightarrow$ $X_{0}$ of a connected complex manifold $X_{0}$ with a deck transformation group $G$ is called a holomorphic $\mathfrak{a}$-function if

(1) $f$ is bounded on subsets $p^{-1}\left(U_{0}\right), U_{0} \Subset X_{0}$, and

(2) for each $x \in X$ the function $G \ni g \mapsto f(g \cdot x)$ belongs to a fixed closed unital subalgebra $\mathfrak{a}:=\mathfrak{a}(G)$ of the algebra $\ell_{\infty}(G)$ of bounded complex functions on $G$ (with pointwise multiplication and sup-norm) that is invariant with respect to the action of $G$ on $\mathfrak{a}$ by right translations:

$$
u \in \mathfrak{a}, \quad g \in G \quad \Rightarrow \quad R_{g} u \in \mathfrak{a},
$$

where $R_{g}(u)(h):=u(h g), h \in G$.

We endow the subalgebra $\mathcal{O}_{\mathfrak{a}}(X) \subset \mathcal{O}(X)$ of holomorphic $\mathfrak{a}$-functions with the Fréchet topology of uniform convergence on subsets $p^{-1}\left(U_{0}\right), U_{0} \Subset X_{0}$.

The model examples of algebras $\mathfrak{a}$ and $\mathcal{O}_{\mathfrak{a}}(X)$ are given in Examples 1.2 and 1.4 below.

In the present paper we obtain analogs of Cartan theorems A and B for coherent-type sheaves on the fibrewise compactification $c_{\mathfrak{a}} X$ of the covering $X$ of 
a Stein manifold $X_{0}$, a topological space having certain features of a complex manifold (see Definition 2.1 below). In our proofs we use some results and methods of the theory of coherent-type sheaves taking values in Banach or Fréchet spaces, pioneered by E. Bishop and L. Bungart [14], [15] and developed further by J. Leiterer [30] (on Stein spaces), L. Lempert [31] (on pseudoconvex subsets of Banach spaces with unconditional bases) and others. The constructions in [31] play particularly important role in our proofs.

In the second part of the work [13] we use our Cartan-type theorems A and B to derive within subalgebra $\mathcal{O}_{\mathfrak{a}}(X)$ the basic results of complex function theory, including holomorphic extension from complex submanifolds, Cousin problems, properties of divisors, corona-type theorems, holomorphic Peter-Weyl-type approximation theorems, Hartogs-type theorems, describe uniqueness sets of holomorphic a-functions, etc.

Example 1.2 (Holomorphic almost periodic functions). The theory of almost periodic functions was created in the 1920s by H. Bohr, who intended to apply it in the study of the distribution of zeros of the zeta-function in the critical strip. Nowadays almost periodic functions are used in many areas of mathematics, including partial differential equations (e.g., KdV equation), harmonic analysis and number theory. A turning point in understanding of the nature of continuous almost periodic functions on $\mathbb{R}$ came with the discovery of the so-called Bohr compactification of $\mathbb{R}$ : according to S. Bochner, it quickly led to "a sobering realization" that the basic results of Bohr's theory on $\mathbb{R}$ can be deduced from Weyl's general theory of continuous functions on compact groups. In the same way, our work demonstrates that the basic results of the theory of holomorphic almost periodic functions in tube domains follow from our Oka-Cartan-type theory. The latter allows us to apply the modern methods of multidimensional complex function theory to holomorphic almost periodic functions, and to obtain new results even in this classical setting.

Let us recall that a function $f \in \mathcal{O}(T)$ on a tube domain $T=\mathbb{R}^{n}+i \Omega \subset \mathbb{C}^{n}$, $\Omega \subset \mathbb{R}^{n}$ is open and convex, is called holomorphic almost periodic if the family of its translates $\{z \mapsto f(z+s), z \in T\}_{s \in \mathbb{R}^{n}}$ is relatively compact in the topology of uniform convergence on tube subdomains $T^{\prime}=\mathbb{R}^{n}+i \Omega^{\prime}, \Omega^{\prime} \Subset \Omega$. The cornerstone of Bohr's theory (see [1]) is his approximation theorem stating that every holomorphic almost periodic function is uniform limit (on tube subdomains $T^{\prime}$ of $T$ ) of exponential polynomials

$$
z \mapsto \sum_{k=1}^{m} c_{k} e^{i\left\langle z, \lambda_{k}\right\rangle}, \quad z \in T, \quad c_{k} \in \mathbb{C}, \quad \lambda_{k} \in \mathbb{R}^{n},
$$

where $\langle\cdot, \cdot\rangle$ is the Hermitian inner product on $\mathbb{C}^{n}$.

The classical approach to the study of holomorphic almost periodic functions exploits the fact that $T$ is the trivial bundle with base $\Omega$ and fibre $\mathbb{R}^{n}$ (e.g., as in the characterization of almost periodic functions in terms of their Jessen functions defined on $\Omega$, see, e.g., [39], [32], [27], [38], [18], [40]). By considering $T$ as a regular 
covering $p: T \rightarrow T_{0}\left(:=p(T) \subset \mathbb{C}^{n}\right)$ with the deck transformation group $\mathbb{Z}^{n}$,

$$
p(z):=\left(e^{i z_{1}}, \ldots, e^{i z_{n}}\right), \quad z=\left(z_{1}, \ldots, z_{n}\right) \in T
$$

(a complex strip covering an annulus if $n=1$ ), we obtain

Theorem 1.3 ([13]). A function $f \in \mathcal{O}(T)$ is almost periodic if and only if $f \in$ $\mathcal{O}_{A P}(T)$.

Here $A P=A P\left(\mathbb{Z}^{n}\right)$ is the algebra of von Neumann's almost periodic functions on group $\mathbb{Z}^{n}$, i.e., those bounded complex functions whose families of translates are relatively compact in the topology of uniform convergence on $\mathbb{Z}^{n}$.

This result enables us to regard holomorphic almost periodic functions on $T$ as:

(a) holomorphic sections of a certain holomorphic Banach vector bundle on $T_{0}$;

(b) holomorphic-like functions on the fibrewise Bohr compactification of the covering $p: T \rightarrow T_{0}$, a topological space having some properties of a complex manifold.

It is interesting to note that already in his monograph [1] H. Bohr uses equally often the aforementioned "trivial fibre bundle" and "regular covering" points of view on a complex strip. We note also that the Bohr compactification of a tube domain $\mathbb{R}^{n}+i \Omega$ in the form $b \mathbb{R}^{n}+i \Omega$, where $b \mathbb{R}^{n}$ is the Bohr compactification of group $\mathbb{R}^{n}$, was used earlier in [16], [17], [21].

Example 1.4. (1) Let $\mathfrak{a}:=\ell_{\infty}(G)$ be the algebra of all bounded complex functions on the deck transformation group $G \cong p^{-1}(x), x \in X_{0}$, of covering $p: X \rightarrow X_{0}$.

By definition, every subalgebra $\mathcal{O}_{\mathfrak{a}}(X) \subset \mathcal{O}_{\ell_{\infty}}(X), \ell_{\infty}:=\ell_{\infty}(G)$.

Algebra $\mathcal{O}_{\ell_{\infty}}(X)$ arises, e.g., in the study of holomorphic $L^{2}$-functions on coverings of pseudoconvex manifolds [22], [3], [6], [29], Caratheodory hyperbolicity (Liouville property) of $X$ [34], [33], corona-type problems for bounded holomorphic functions on $X[2]$. Earlier, some methods similar to those developed in the article were elaborated for algebra $\mathcal{O}_{\ell_{\infty}}(X)$ in [2]-[5] in connection with coronatype problems for some subalgebras of bounded holomorphic functions on coverings of bordered Riemann surfaces, Hartogs-type theorems, integral representation of holomorphic functions of slow growth on coverings of Stein manifolds, etc; that work was motivated by the fact that if $X_{0}$ is compact, then $\mathcal{O}_{\ell_{\infty}}(X)=H^{\infty}(X)$, the algebra of all bounded holomorphic functions on $X$ (the most important cases are when $X$ is the unit ball or polydisk in $\mathbb{C}^{n}$ ).

A confirmation of potential productivity of the sheaf-theoretic approach to the corona problem for $H^{\infty}$ comes from the recent papers [9], [10] on Banach-valued holomorphic functions on the unit disk $\mathbb{D} \subset \mathbb{C}$ having relatively compact images.

(2) Let $\mathfrak{a}:=c(G)$ (with card $G=\infty$ ) be the subalgebra of bounded complex functions on $G$ that admit continuous extensions to the one-point compactification of $G$ (here we consider $G$ equipped with discrete topology). Then $\mathcal{O}_{c}(X)$ consists of holomorphic functions having fibrewise limits at 'infinity'.

Acknowledgement. We thank Professors T. Bloom, L. Lempert, T. Ohsawa, R. Shafikov and Y.-T. Siu for their interest to this work. 


\section{Main results}

In some cases the maximal ideal space of algebra $\mathcal{O}_{\mathfrak{a}}(X)$ may be presented as a 'fibrewise compactification' $c_{\mathfrak{a}} X$ of the covering $p: X \rightarrow X_{0}$. Now we briefly present this construction referring to Section 4 for further details.

Let $M_{\mathfrak{a}}$ denote the maximal ideal space of algebra $\mathfrak{a}$, i.e., the space of all characters of $\mathfrak{a}$ endowed with weak ${ }^{*}$ topology (of $\mathfrak{a}^{*}$ ). The space $M_{\mathfrak{a}}$ is compact and Hausdorff, and every element $f$ of $\mathfrak{a}$ determines a function $\hat{f} \in C\left(M_{\mathfrak{a}}\right)$ by the formula

$$
\hat{f}(\eta):=\eta(f), \quad \eta \in M_{\mathfrak{a}} .
$$

Since algebra $\mathfrak{a}$ is uniform (i.e., $\left\|f^{2}\right\|=\|f\|^{2}$ ) and hence is semi-simple, the homomorphism^: $\mathfrak{a} \rightarrow C\left(M_{\mathfrak{a}}\right)$ (called the Gelfand transform) is an isometric embedding (see, e.g., [19]). We have a continuous map $j=j_{\mathfrak{a}}: G \rightarrow M_{\mathfrak{a}}$ defined by associating to each point in $G$ its point evaluation homomorphism in $M_{\mathfrak{a}}$. This map is an injection if and only if algebra $\mathfrak{a}$ separates points of $G$.

Let $\hat{G}_{\mathfrak{a}}$ denote the closure of $j(G)$ in $M_{\mathfrak{a}}$. If algebra $\mathfrak{a}$ is self-adjoint (i.e., closed with respect to complex conjugation), then ${ }^{\wedge}: \mathfrak{a} \rightarrow C\left(M_{\mathfrak{a}}\right)$ is an isomorphism and hence $\hat{G}_{\mathfrak{a}}=M_{\mathfrak{a}}$. The (right) action of group $G$ on itself by right multiplication induces the right action of $G$ on $M_{\mathfrak{a}}$ by the formula

$$
\hat{R}_{g}(\eta)(f):=\eta\left(R_{g}(f)\right), \quad \eta \in M_{\mathfrak{a}}, f \in \mathfrak{a}, g \in G .
$$

Then

$$
\hat{R}_{g}(j(h))=j(h g), \quad h, g \in G .
$$

The regular covering $p: X \rightarrow X_{0}$ can be viewed as a principal fibre bundle on $X_{0}$ with structure group $G$, that is there exists an open cover $\left(U_{0, \gamma}\right)_{\gamma \in \Gamma}$ of $X_{0}$ and a locally constant cocycle $c=\left\{c_{\delta \gamma}: U_{0, \gamma} \cap U_{0, \delta} \rightarrow G\right\}_{\delta, \gamma \in \Gamma}$ so that the covering $p: X \rightarrow X_{0}$ can be obtained from the disjoint union $\bigsqcup_{\gamma} U_{0, \gamma} \times G$ by the identification

$$
U_{0, \delta} \times G \ni(x, g) \sim\left(x, g \cdot c_{\delta \gamma}(x)\right) \in U_{0, \gamma} \times G \quad \text { for all } x \in U_{0, \gamma} \cap U_{0, \delta},
$$

where projection $p$ is induced by the projections $U_{0, \gamma} \times G \rightarrow U_{0, \gamma}$ (see, e.g., [26]).

Definition 2.1. The fibrewise (a-) compactification $\bar{p}: c_{\mathfrak{a}} X \rightarrow X_{0}$ is the fibre bundle on $X_{0}$ with fibre $\hat{G}_{\mathfrak{a}}$ associated to the principal bundle $p: X \rightarrow X_{0}$, i.e., $c_{\mathfrak{a}} X$ is obtained from the disjoint union $\bigsqcup_{\gamma} U_{0, \gamma} \times \hat{G}_{\mathfrak{a}}$ by the identification

$$
U_{0, \gamma} \times \hat{G}_{\mathfrak{a}} \ni(x, \omega) \sim\left(x, \hat{R}_{c_{\delta \gamma}(x)}(\omega)\right) \in U_{0, \delta} \times \hat{G}_{\mathfrak{a}}, \quad \text { for all } x \in U_{0, \gamma} \cap U_{0, \delta},
$$

where $\bar{p}$ is induced by projections $U_{0, \gamma} \times \hat{G}_{\mathfrak{a}} \rightarrow U_{0, \gamma}$.

In [13], Theorem 5.18, we show that if subalgebra $\mathfrak{a}$ is self-adjoint and $X_{0}$ is a Stein manifold, then the maximal ideal space of algebra $\mathcal{O}_{\mathfrak{a}}(X)$ (i.e., the set of non-zero continuous complex homomorphisms of $\mathcal{O}_{\mathfrak{a}}(X)$ endowed with weak* topology of $\mathcal{O}_{\mathfrak{a}}(X)^{*}$ ) is homeomorphic to $c_{\mathfrak{a}} X$. (In particular, this is applied to algebras of Examples 1.2 and 1.4.) 
Next, there exists a continuous map

$$
\iota=\iota_{\mathfrak{a}}: X \rightarrow c_{\mathfrak{a}} X
$$

induced by the equivariant (with respect to the corresponding actions of $G$ on $G$ and $\hat{G}_{\mathfrak{a}}$ ) map $j$. Clearly, $\iota(X)$ is dense in $c_{\mathfrak{a}} X$ (thus, if $X_{0}$ is Stein and $\mathfrak{a}$ is selfadjoint, we have a corona-type theorem for algebra $\mathcal{O}_{\mathfrak{a}}(X)$ as its maximal ideal space is homeomorphic to $c_{\mathfrak{a}} X$, see [13]). The map $\iota$ is an injection if and only if $\mathfrak{a}$ separates points of $G$.

Definition 2.2. A function $f \in C\left(c_{\mathfrak{a}} X\right)$ is called holomorphic if its pullback $\iota^{*} f$ is holomorphic on $X$. The algebra of functions holomorphic on $c_{\mathfrak{a}} X$ is denoted by $\mathcal{O}\left(c_{\mathfrak{a}} X\right)$.

Proposition 2.3. The following is true:

(1) A function $f$ in $\mathcal{O}_{\mathfrak{a}}(X)$ determines a unique function $\hat{f}$ in $\mathcal{O}\left(c_{\mathfrak{a}} X\right)$ such that $\iota^{*} \hat{f}=f$. Thus, there is a continuous embedding $\mathcal{O}_{\mathfrak{a}}(X) \hookrightarrow \mathcal{O}\left(c_{\mathfrak{a}} X\right)$.

(2) If $\mathfrak{a}$ is self-adjoint, then the correspondence $f \mapsto \hat{f}$ determines an isomorphism of algebras: $\mathcal{O}_{\mathfrak{a}}(X) \cong \mathcal{O}\left(c_{\mathfrak{a}} X\right)$.

So, for $\mathfrak{a}$ self-adjoint we can work with algebra $\mathcal{O}\left(c_{\mathfrak{a}} X\right)$ instead of $\mathcal{O}_{\mathfrak{a}}(X)$.

Definition 2.4. Let $U \subset c_{\mathfrak{a}} X$ be an open subset. A function $f \in C(U)$ is called holomorphic if $\iota^{*} f \in \mathcal{O}\left(\iota^{-1}(U)\right)$. The algebra of functions holomorphic on $U$ is denoted by $\mathcal{O}(U)$.

Thus, we obtain the structure sheaf $\mathcal{O}:=\mathcal{O}_{c_{\mathfrak{a}} X}$ of germs of holomorphic functions on $c_{\mathfrak{a}} X$. Now, a coherent sheaf $\mathcal{A}$ on $c_{\mathfrak{a}} X$ is a sheaf of modules over $\mathcal{O}$ such that every point in $c_{\mathfrak{a}} X$ has an open neighbourhood $U$ over which, for every $N \geq 1$, there is a free resolution of $\mathcal{A}$ of length $N$, i.e., an exact sequence of sheaves of modules of the form

$$
\left.\left.\left.\left.\mathcal{O}^{m_{N}}\right|_{U} \stackrel{\varphi_{N-1}}{\longrightarrow} \ldots \stackrel{\varphi_{2}}{\longrightarrow} \mathcal{O}^{m_{2}}\right|_{U} \stackrel{\varphi_{1}}{\longrightarrow} \mathcal{O}^{m_{1}}\right|_{U} \stackrel{\varphi_{0}}{\longrightarrow} \mathcal{A}\right|_{U} \longrightarrow 0
$$

where $\varphi_{i}, 0 \leq i \leq N-1$, are homomorphisms of sheaves of $\mathcal{O}$-modules.

If $X=X_{0}$ and $p=\mathrm{Id}$, then this definition gives the classical definition of a coherent sheaf on a complex manifold.

Our main results are stated as follows.

Let $X_{0}$ be a Stein manifold, $\mathfrak{a}$ be self-adjoint, $\mathcal{A}$ be a coherent sheaf on $c_{\mathfrak{a}} X$.

Theorem 2.5 (Cartan A). Each stalk ${ }_{x} \mathcal{A}\left(x \in c_{\mathfrak{a}} X\right)$ is generated as an ${ }_{x} \mathcal{O}$-module by global sections of sheaf $\mathcal{A}$ over $c_{\mathfrak{a}} X$.

Theorem 2.6 (Cartan B). Čech cohomology groups $H^{i}\left(c_{\mathfrak{a}} X, \mathcal{A}\right)=0$ for all $i \geq 1$. 
Note that the classical proof of Cartan theorems A and B on complex manifolds does not work in our case, in particular, because of absence of the Oka coherence lemma and since the fibre $\hat{G}_{\mathfrak{a}}$ being an arbitrary compact Hausdorff space does not admit open covers by contractible sets as required for the proof of the classical Cartan lemma. Instead, we paste together free resolutions (2.4) of a coherent sheaf $\mathcal{A}$ first over sets $\bar{p}^{-1}\left(U_{0}\right)$, with $U_{0} \subset X_{0}$ being open simply connected, using continuous partition of unity in $C\left(\hat{G}_{\mathfrak{a}}\right)(\cong \mathfrak{a})$ and employing some constructions of L. Lempert [31]. Then we paste the obtained free resolutions of $\mathcal{A}$ on sets $\bar{p}^{-1}\left(U_{0}\right)$ to get free resolutions of $\mathcal{A}$ over preimages by $\bar{p}$ of relatively compact open subsets of $X_{0}$ forming an exhaustion of $c_{\mathfrak{a}} X$. At this stage, we use the results of J. Leiterer [30] on Banach-valued coherent sheaves on Stein manifolds. Finally, having these free resolutions of $\mathcal{A}$, we complete the proof of Theorems 2.5 and 2.6 as in the classical case.

Remark 2.7. (1) An important example of a coherent sheaf on $c_{\mathfrak{a}} X$ is given by the sheaf of ideals of germs of holomorphic functions vanishing on a complex submanifold of $c_{\mathfrak{a}} X$. To define the latter, we will need the following notation. Let $U_{0} \subset X_{0}$ be open and simply connected, $K \subset \hat{G}_{\mathfrak{a}}$ be open. We denote by $\bar{\psi}_{U_{0}}$ the trivialization $\bar{p}^{-1}\left(U_{0}\right) \rightarrow U_{0} \times \hat{G}_{\mathfrak{a}}$ (see Definition 2.1), and define

$$
\hat{\Pi}\left(U_{0}, K\right)=\bar{\psi}_{U_{0}}^{-1}(K) \subset c_{\mathfrak{a}} X .
$$

In what follows, we identify $\hat{\Pi}\left(U_{0}, K\right)$ with $U_{0} \times K$ (see subsection 4.2 for details).

Definition 2.8. A closed subset $Y \subset c_{\mathfrak{a}} X$ is called a complex submanifold of codimension $k$ if for every $x \in Y$ there exist a neighbourhood $U=\hat{\Pi}\left(U_{0}, K\right) \subset c_{\mathfrak{a}} X$ of $x$ and functions $h_{1}, \ldots, h_{k} \in \mathcal{O}(U)$ such that

(a) $Y \cap U=\left\{x \in U: h_{1}(x)=\cdots=h_{k}(x)=0\right\}$;

(b) The rank of map $z \mapsto\left(h_{1}(z, \omega), \ldots, h_{k}(z, \omega)\right)$ is $k$ at each point $(z, \omega) \in Y \cap U$.

We use coherence of the sheaf of ideals of $Y$ together with Theorem 2.6 in [13] to obtain results on interpolation within algebra $\mathcal{O}_{\mathfrak{a}}(X)$. In the same paper we extend Cartan theorems A and B to work with coherent-type sheaves on complex submanifolds of $c_{\mathfrak{a}} X$.

(2) The assumption that subalgebra $\mathfrak{a}$ is self-adjoint is essential for our proofs of Theorems 2.5 and 2.6. Nevertheless, using a technique different from that based on Theorem 2.6, we show in [13] that the problem of interpolation within algebra $\mathcal{O}_{\mathfrak{a}}(X)$ can be solved (although for a restrictive class of sets) without the latter assumption.

(3) An interesting example of the algebra of holomorphic $\mathfrak{a}$-functions with $\mathfrak{a}$ not self-adjoint is given by Bohr's holomorphic almost periodic functions on an open horizontal strip $T \subset \mathbb{C}$ (see Example 1.2) whose restrictions to each fibre $p^{-1}(x) \cong \mathbb{Z}$ belong to the subalgebra $A P_{+}(\mathbb{Z})$ of the von Neumann almost periodic functions on $\mathbb{Z}$ with positive spectra, i.e., those functions on $\mathbb{Z}$ that admit uniform approximation by exponential polynomials $\sum_{k=1}^{m} c_{k} e^{i \lambda_{k} t}(t \in \mathbb{Z})$ with $\lambda_{k} \geq 0$ (see [8]). One can show that the maximal ideal space of algebra $\mathcal{O}_{A P_{+}}(T)$ can 
be presented as inverse limit of an inverse limiting system of holomorphic fibre bundles over an annulus whose fibres are biholomorphic to disjoint unions of open polydisks, see Example 4.2 below.

\section{Examples}

Example 3.1 (Examples of algebras $\mathfrak{a}$ ). In addition to algebras $\ell_{\infty}(G), c(G)$, $A P\left(\mathbb{Z}^{n}\right)$ (see Examples 1.2 and 1.4) we mention the following important examples of self-adjoint subalgebras of $\ell_{\infty}(G)$ invariant with respect to actions of $G$ by right translations.

(1) If a group $G$ is residually finite (respectively, residually nilpotent), i.e., for each element $g \in G, g \neq e$, there exists a normal subgroup $G_{g} \not \supset g$ such that $G / G_{g}$ is finite (respectively, nilpotent), we consider the closed subalgebra $\hat{\ell}_{\infty}(G) \subset \ell_{\infty}(G)$ generated by pullbacks to $G$ of algebras $\ell_{\infty}\left(G / G_{g}\right)$ for all $G_{g}$ as above.

(2) Recall that a bounded complex function $f$ on a (discrete) group $G$ is called almost periodic if the families of its left and right translates

$$
\{t \mapsto f(s t)\}_{s \in G}, \quad\{t \mapsto f(t s)\}_{s \in G}
$$

are relatively compact in $\ell_{\infty}(G)$ (J. von Neumann [37]). (It was proved in [35] that the relative compactness of either the left of the right family of translates already gives almost periodicity.) The algebra of almost periodic functions on $G$ is denoted by $A P(G)$.

The basic examples of almost periodic functions on $G$ are matrix elements of finite-dimensional irreducible unitary representations of $G$.

Recall that a topological group $G$ is called maximally almost periodic if its finite-dimensional irreducible unitary representations separate points of $G$. Equivalently, $G$ is maximally almost periodic if and only if it admits a (continuous) monomorphism into a compact topological group.

Any residually finite discrete group $G$ belongs to this class. In particular, $\mathbb{Z}^{n}$, finite groups, free groups, finitely generated nilpotent groups, pure braid groups, fundamental groups of three dimensional manifolds are maximally almost periodic.

We denote by $A P_{0}(G) \subset A P(G)$ the space of functions

$$
t \mapsto \sum_{k=1}^{m} c_{k} \sigma_{i j}^{k}(t), \quad t \in G, c_{k} \in \mathbb{C}, \sigma^{k}=\left(\sigma_{i j}^{k}\right),
$$

where $\sigma^{k}, 1 \leq k \leq m$, are finite-dimensional irreducible unitary representations of $G$. The von Neumann approximation theorem states that $A P_{0}(G)$ is dense in $A P(G)[37]$.

In particular, algebra $A P\left(\mathbb{Z}^{n}\right)$ of almost periodic functions on $\mathbb{Z}^{n}$ contains as a dense subset the set of exponential polynomials $t \mapsto \sum_{k=1}^{m} c_{k} e^{i\left\langle\lambda_{k}, t\right\rangle}, t \in \mathbb{Z}^{n}$, $c_{k} \in \mathbb{C}, \lambda_{k} \in \mathbb{R}^{n}$. Here $\langle\cdot, \cdot\rangle$ is the standard inner product on $\mathbb{R}^{n}$.

(3) Algebra $A P_{\mathbb{Q}}\left(\mathbb{Z}^{n}\right)$ of almost periodic functions on $\mathbb{Z}^{n}$ with rational spectra. This is the subalgebra of $A P\left(\mathbb{Z}^{n}\right)$ generated (over $\mathbb{C}$ ) by functions of the form $t \mapsto e^{i\langle\lambda, t\rangle}$ with $\lambda \in \mathbb{Q}$. 
(4) Suppose that the covering $p: X \rightarrow X_{0}$ is not regular. Still, we can define algebras $\mathcal{O}_{\ell_{\infty}}(X)$ and $\mathcal{O}_{c}(X)$ and include them in the framework of our theory by lifting them (by the pullback of the covering map) to the universal covering of $X_{0}$.

Example 3.2 (Holomorphic almost periodic functions). Elements of $\mathcal{O}_{A P}(X)$, where $X \rightarrow X_{0}$ is a regular covering of a connected complex manifold $X_{0}$ with a deck transformation group $G$ and $A P:=A P(G)$, see Example 3.1 (2), are called holomorphic almost periodic functions. Equivalently, a function $f \in \mathcal{O}(X)$ is called holomorphic almost periodic if each orbit $\{g \cdot x\}_{g \in G} \subset X$ has an open neighbourhood $U \subset X$, invariant with respect to the action of $G$ on $X$, such that the family of translates $\{z \mapsto f(g \cdot z), z \in U\}_{g \in G}$ is relatively compact in the topology of uniform convergence on $U$ (see [11] for the proof of the equivalence).

This is a variant of the definition in [42], where $G$ is taken to be the group of all biholomorphic automorphisms of the complex manifold $X$. An interesting result in [41] states that on Siegel domains of the second kind there are no nonconstant holomorphic almost periodic functions in the sense of [42] (although on Siegel domains of the first kind, i.e., on tube domains in $\mathbb{C}^{n}$, such holomorphic almost periodic functions even separate points). A similar result holds for the algebra $\mathcal{O}_{A P}(X)$; for instance, if $X_{0}$ is a compact complex manifold, then all holomorphic almost periodic functions on $X$ are constant, see Theorem 2.3 in [11].

Example 3.3 (Compactification of deck transformation group $G$ ). (1) Let $\mathfrak{a}:=$ $c(G)$, card $G=\infty$ (see Example $1.4(2))$. Then $\hat{G}_{c}, c:=c(G)$, is the one-point compactification of $G$.

(2) Let $\mathfrak{a}=A P(G)(=: A P)$ (see Example $3.1(2))$. Then $\hat{G}_{A P}$ is homeomorphic to a compact topological group $b G$, called the Bohr compactification of $G$, uniquely determined by the universal property: there exists a homomorphism $\mu: G \rightarrow b G$ such that for any compact topological group $H$ and any homomorphism $\nu: G \rightarrow H$ there exists a continuous homomorphism $\tilde{\nu}: b G \rightarrow H$ such that the following diagram

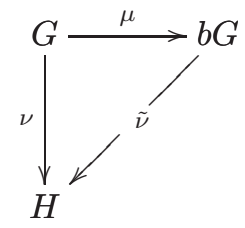

is commutative.

Applying this property to unitary groups $H:=U_{n}, n \geq 1$, we obtain that group $G$ is maximally almost periodic (see Example $3.1(2)$ ) if and only if $\mu$ is a monomorphism.

The universal property implies that there exists a bijection between sets of finite-dimensional irreducible unitary representations of $G$ and $b G$. It turn, the Peter-Weyl theorem for $C(b G)$ and the von Neumann approximation theorem for $A P(G)$ (see Example $3.1(2)$ ) imply that $A P(G) \cong C(b G)$. Therefore, $b G$ is homeomorphic to the maximal ideal space $M_{A P(G)}$ of algebra $A P(G)$ and $\mu(G)$ is dense in $b G$. Under this homeomorphism, the set $j(G)$ (see Section 2 for its 
definition) is identified with the subgroup $\mu(G) \subset b G$. In case $G$ is maximally almost periodic, we will identify $G$ with $\mu(G) \subset b G$ by means of $\mu$ so that the action of $G$ on $\hat{G}_{A P}:=M_{A P(G)}$ coincides with that of $G$ on $b G$ by right translations.

By the Peter-Weyl theorem, the group $b G$ can be presented as inverse limit of an inverse system of finite-dimensional compact Lie groups. In particular, the Bohr compactification $b \mathbb{Z}$ of the group of integers $\mathbb{Z}$ is inverse limit of an inverse system of compact abelian Lie groups $\mathbb{T}^{k} \times \oplus_{l=1}^{m} \mathbb{Z} /\left(n_{l} \mathbb{Z}\right), k, m, n_{l} \in \mathbb{N}$, where $\mathbb{T}^{k}:=\left(\mathbb{S}^{1}\right)^{k}$ is the real $k$-torus. It follows that $b \mathbb{Z}$ is disconnected and has infinite covering dimension. The limit homomorphisms $b \mathbb{Z} \rightarrow \mathbb{T}^{k} \times \oplus_{l=1}^{m} \mathbb{Z} /\left(n_{l} \mathbb{Z}\right)$ are defined by finite families of characters $\mathbb{Z} \rightarrow \mathbb{S}^{1}$. For instance, let $\lambda_{1}, \lambda_{2} \in \mathbb{R} \backslash \mathbb{Q}$ be linearly independent over $\mathbb{Q}$ and $\chi_{\lambda_{i}}: \mathbb{Z} \rightarrow \mathbb{S}^{1}, \chi_{\lambda_{i}}(n):=e^{2 \pi i \lambda_{i} n}, i=1,2$, be the corresponding characters. Then the map $\left(\chi_{\lambda_{1}}, \chi_{\lambda_{2}}\right): \mathbb{Z} \rightarrow \mathbb{T}^{2}$ is extended by continuity to a continuous surjective homomorphism $b \mathbb{Z} \rightarrow \mathbb{T}^{2}$. If $\lambda_{1}, \lambda_{2}$ are linearly dependent over $\mathbb{Q}$, then the corresponding extended homomorphism has image in $\mathbb{T}^{2}$ isomorphic to $\mathbb{S}^{1} \times \mathbb{Z} /(m \mathbb{Z})$ for some $m \in \mathbb{N}$.

(3) Let $\mathfrak{a}=A P_{\mathbb{Q}}\left(\mathbb{Z}^{n}\right)$ (see Example $3.1(3)$ ). Then $\hat{G}_{A P_{\mathbb{Q}}\left(\mathbb{Z}^{n}\right)}$ is homeomorphic to the profinite completion of group $\mathbb{Z}^{n}$; it is defined as inverse limit of an inverse system of groups $\oplus_{l=1}^{m} \mathbb{Z} /\left(n_{l} \mathbb{Z}\right), m, n_{l} \in \mathbb{N}$. It follows that covering dimension of $\hat{G}_{A P_{\mathbb{Q}}\left(\mathbb{Z}^{n}\right)}$ is zero.

(4) Let $\mathfrak{a}=\ell_{\infty}(G)\left(=: \ell_{\infty}\right)$ (see Example 1.4 (1)). Then $\hat{G}_{\ell_{\infty}} \cong \beta G$, the StoneČech compactification of group $G$. Covering dimension of $\hat{G}_{\ell_{\infty}}$ is zero (see, e.g., Theorem 9-5 in [36]).

\section{Structure of fibrewise compactification $c_{\mathfrak{a}} X$}

\subsection{Set structure of $c_{\mathfrak{a}} X$}

As a set, $c_{\mathfrak{a}} X$ is the disjoint union of connected complex manifolds, each is a covering of $X_{0}$. Indeed, let $\Upsilon:=\hat{G}_{\mathfrak{a}} / G$ be the set of orbits of elements of $\hat{G}_{\mathfrak{a}}$ by the (right) action of $G$; since any orbit $H \in \Upsilon$ is invariant with respect to the action of $G$, we may consider the associated to the action of $G$ on $H$ fibre bundle $p_{H}: X_{H} \rightarrow X_{0}$ with fibre $H$. We assume that $H$ is endowed with discrete topology. Then $p_{H}: X_{H} \rightarrow X_{0}$ is an unbranched covering of $X_{0}$ (in general non-regular). Since $X$ is connected and $X$ is a covering of $X_{H}$, the complex manifold $X_{H}$ is connected as well. For each $H \in \Upsilon$ we have the natural continuous injective map

$$
\iota_{H}: X_{H} \hookrightarrow c_{\mathfrak{a}} X
$$

determined by (equivariant with respect to the action of $G$ ) inclusion $H \hookrightarrow \hat{G}_{\mathfrak{a}}$. We denote $\hat{X}_{H}:=\iota_{H}\left(X_{H}\right)$. In view of $(2.1)$, we have $j(G) \in \Upsilon$. Hence, if $j$ is injective, then $X=X_{j(G)}$ and $\iota=\iota_{j(G)}($ see $(2.3))$.

It follows that, as a set,

$$
c_{\mathfrak{a}} X=\bigsqcup_{H \in \Upsilon} \iota_{H}\left(X_{H}\right)
$$


Example 4.1. $\hat{G}_{c}:=G \cup\{\infty\}$, card $G=\infty$, is the one-point compactification of $G$, and the action of $G$ on $\hat{G}_{c}$ fixes point $\infty$, so $\Upsilon=\{\{G\},\{\infty\}\}$. It follows that, as a set, $c_{\mathfrak{a}} X$ is the disjoint union of two spaces homeomorphic to $X$ and $X_{0}$, respectively.

Example 4.2. Let $\mathfrak{a}=A P(G)$ and $G$ be maximally almost periodic. In what follows, we assume that $\hat{G}_{A P}$ is endowed with the group structure of the Bohr compactification $b G$, see Example $3.3(2)$.

Since $G$ is a subgroup of $b G$, every orbit $H \in \Upsilon$ is a right coset of $G$ in $b G$, $X_{H}=X$ for all $H \in \Upsilon$, and each set $\hat{X}_{H}$ is dense in $c_{A P} X$.

The fibre bundle $c_{A P} X$ can be presented as inverse limit of an inverse system of smooth fibre bundles on $X_{0}$. Indeed, by the Peter-Weil theorem, $b G$ can be presented as inverse limit of an inverse system of finite-dimensional compact Lie groups $\left\{G_{s}\right\}_{s \in S}$ (see Example 3.3(2)). By $\pi_{s}: b G \rightarrow G_{s}$ we denote the corresponding limit homomorphisms. The right action of $G$ on $b G$ determines an action $r_{s}$ of $G$ on $G_{s}, r_{s}(g)(h):=h \cdot \pi_{s}(g), g \in G, h \in G_{s}$. Let $p_{s}: X_{s} \rightarrow X_{0}$ be the associated to $r_{s}$ fibre bundle on $X_{0}$ with fibre $G_{s}$. Then $X_{s}$ has a smooth manifold structure and inverse limit along $\left\{G_{s}\right\}_{s \in S}$ determines inverse limit along the corresponding inverse system $\left\{X_{s}\right\}_{s \in S}$ which is homeomorphic to $c_{A P} X$.

Example 4.3. Let $\mathfrak{a}=A P_{\mathbb{Q}}\left(\mathbb{Z}^{n}\right)$. Since covering dimension of $\hat{G}_{A P_{\mathbb{Q}}\left(\mathbb{Z}^{n}\right)}$ is zero (see Example $3.3(3)$ ), covering dimension of $c_{A P_{\mathbb{Q}}\left(\mathbb{Z}^{n}\right)} X$ is equal to $\operatorname{dim}_{\mathbb{R}} X_{0}$.

Example 4.4. Let $\mathfrak{a}=\ell_{\infty}(G)$. Then $\hat{G}_{\ell_{\infty}} \cong \beta G$, the Stone-Čech compactification of group $G$. Since covering dimension of $\hat{G}_{\ell_{\infty}}$ is zero, covering dimension of $c_{\ell_{\infty}} X$ coincides with real dimension of $X_{0}$.

It is easy to see that $c_{\ell_{\infty}} X$ is the maximal fibrewise compactification of covering $X \rightarrow X_{0}$ in the sense that if $\mathfrak{a} \subset \ell_{\infty}(G)$ is a (closed) subalgebra, then there exists a surjective bundle morphism $c_{\ell_{\infty}} X \rightarrow c_{\mathfrak{a}} X$. Indeed, let $\kappa: \hat{G}_{\ell_{\infty}} \rightarrow \hat{G}_{\mathfrak{a}}$ be a continuous surjective map adjoint to the inclusion $\mathfrak{a} \hookrightarrow \ell_{\infty}(G)$; since $\kappa$ is equivariant with respect to the corresponding (right) actions of $G$, it determines the required surjective bundle morphism.

\subsection{Complex structure on $c_{\mathfrak{a}} X$}

A function $f \in C(U)$ on an open subset $U \subset c_{\mathfrak{a}} X$ is called holomorphic if $\iota^{*} f$ is holomorphic on $\iota^{-1}(U) \subset X$ in the usual sense.

Let $U_{0} \subset X_{0}$ be open. A function $f \in C(U)$ on an open subset $U \subset U_{0} \times \hat{G}_{\mathfrak{a}}$ is called holomorphic if $\tilde{j}^{*} f, \tilde{j}:=\operatorname{Id} \times j: U_{0} \times G \rightarrow U_{0} \times \hat{G}_{\mathfrak{a}}$, is holomorphic on the open subset $\tilde{j}^{-1}(U)$ of the complex manifold $U_{0} \times G$ (see Section 2 for the definition of the map $j$ ).

For sets $U$ as above, let $\mathcal{O}(U)$ denote the algebra of holomorphic functions on $U$ endowed with the topology of uniform convergence on compact subsets of $U$. Clearly, $f \in C\left(c_{\mathfrak{a}} X\right)$ belongs to $\mathcal{O}\left(c_{\mathfrak{a}} X\right)$ if and only if each point in $c_{\mathfrak{a}} X$ has an open neighbourhood $U$ such that $\left.f\right|_{U} \in \mathcal{O}(U)$, see Definition 2.2.

By $\mathcal{O}_{U}$ we denote the sheaf of germs of holomorphic functions on $U$. 
The category $\mathcal{M}$ of ringed spaces of the form $\left(U, \mathcal{O}_{U}\right)$, where $U$ is either an open subset of $c_{\mathfrak{a}} X$ and $X$ is a regular covering of a complex manifold $X_{0}$ or is an open subset of $U_{0} \times \hat{G}_{\mathfrak{a}}$ with $U_{0} \subset X_{0}$ open, contains in particular complex manifolds.

Definition 4.5. A morphism of two objects in $\mathcal{M}$, that is, a map $F \in C\left(U_{1}, U_{2}\right)$, where $\left(U_{i}, \mathcal{O}_{U_{i}}\right) \in \mathcal{M}, i=1,2$, such that $F^{*} \mathcal{O}_{U_{2}} \subset \mathcal{O}_{U_{1}}$, is called a holomorphic map.

The collection of holomorphic maps $F: U_{1} \rightarrow U_{2},\left(U_{i}, \mathcal{O}_{U_{i}}\right) \in \mathcal{M}, i=1,2$, is denoted by $\mathcal{O}\left(U_{1}, U_{2}\right)$. If $F \in \mathcal{O}\left(U_{1}, U_{2}\right)$ has inverse $F^{-1} \in \mathcal{O}\left(U_{2}, U_{1}\right)$, then $F$ is called a biholomorphism.

The next result shows that, in a sense, the holomorphic structure on $c_{\mathfrak{a}} X$ is concentrated in 'horizontal layers' $\hat{X}_{H} \subset c_{\mathfrak{a}} X(H \in \Upsilon)$.

Theorem 4.6. For a connected complex manifold $M$ and a map $F \in \mathcal{O}\left(M, c_{\mathfrak{a}} X\right)$ there exists $H \in \Upsilon$ such that $F(M) \subset \hat{X}_{H}$.

If covering dimension of $\hat{G}_{\mathfrak{a}}$ is zero (see Examples 4.3 and 4.4 ), then the assertion of Theorem 4.6 holds true even for continuous maps, i.e., for every $F \in C\left(M, c_{\mathfrak{a}} X\right)$ there exists $H \in \Upsilon$ such that $F(M) \subset \hat{X}_{H}$ (see the argument in the proof of Theorem $1.2(\mathrm{~d})$ in [7]).

Further, over each simply connected open subset $U_{0} \subset X_{0}$ there exists a biholomorphic trivialization $\psi=\psi_{U_{0}}: p^{-1}\left(U_{0}\right) \rightarrow U_{0} \times G$ of covering $p: X \rightarrow X_{0}$ which is a morphism of fibre bundles with fibres $G$. Then there exists a biholomorphic trivialization $\bar{\psi}=\bar{\psi}_{U_{0}}: \bar{p}^{-1}\left(U_{0}\right) \rightarrow U_{0} \times \hat{G}_{\mathfrak{a}}$ of bundle $c_{\mathfrak{a}} X$ over $U_{0}$, which is a morphism of fibre bundles with fibre $\hat{G}_{\mathfrak{a}}$, such that the following diagram

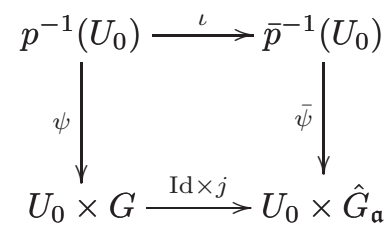

is commutative.

For a given subset $S \subset G$ we denote

$$
\Pi\left(U_{0}, S\right):=\psi^{-1}\left(U_{0} \times S\right)
$$

and identify $\Pi\left(U_{0}, S\right)$ with $U_{0} \times S$ where appropriate (here $\Pi\left(U_{0}, G\right)=p^{-1}\left(U_{0}\right)$ ).

For a subset $K \subset \hat{G}_{\mathfrak{a}}$ we denote

$$
\hat{\Pi}\left(U_{0}, K\right)\left(=\hat{\Pi}_{\mathfrak{a}}\left(U_{0}, K\right)\right):=\bar{\psi}^{-1}\left(U_{0} \times K\right) .
$$

A pair of the form $\left(\hat{\Pi}\left(U_{0}, K\right), \bar{\psi}\right)$ will be called a coordinate chart for $c_{\mathfrak{a}} X$. Similarly, sometimes we identify $\hat{\Pi}\left(U_{0}, K\right)$ with $U_{0} \times K$. If $K \subset \hat{G}_{\mathfrak{a}}$ is open, then, by our definitions, $\bar{\psi}^{*}: \mathcal{O}\left(U_{0} \times K\right) \rightarrow \mathcal{O}\left(\hat{\Pi}\left(U_{0}, K\right)\right)$ is an isomorphism of (topological) algebras. 


\subsection{Basis of topology on $c_{\mathfrak{a}} X$}

We denote by $\mathfrak{Q}$ the basis of topology of $\hat{G}_{\mathfrak{a}}$ consisting of sets of the form

$$
\left\{\eta \in \hat{G}_{\mathfrak{a}}: \max _{1 \leq i \leq m}\left|h_{i}(\eta)-h_{i}\left(\eta_{0}\right)\right|<\varepsilon\right\}
$$

for $\eta_{0} \in \hat{G}_{\mathfrak{a}}, h_{1}, \ldots, h_{m} \in C\left(\hat{G}_{\mathfrak{a}}\right)$, and $\varepsilon>0$.

The fibrewise compactification $c_{\mathfrak{a}} X$ is a paracompact Hausdorff space (as a fibre bundle with a paracompact base and a compact fibre); thus, $c_{\mathfrak{a}} X$ is a normal space.

It is easy to see that the family

(4.4) $\mathfrak{B}:=\left\{\hat{\Pi}\left(V_{0}, L\right) \subset c_{\mathfrak{a}} X: V_{0}\right.$ is open simply connected in $X_{0}$ and $\left.L \in \mathfrak{Q}\right\}$. forms a basis of topology of $c_{\mathfrak{a}} X$.

\subsection{Coherent sheaves on $c_{\mathfrak{a}} X$}

A sheaf of modules on an open subset $U \subset c_{\mathfrak{a}} X$ over $\left.\mathcal{O}\right|_{U}$ will be called an analytic sheaf. A homomorphism between analytic sheaves will be called an analytic homomorphism.

Recall that a coherent sheaf $\mathcal{A}$ on $c_{\mathfrak{a}} X$ is an analytic sheaf such that every point in $c_{\mathfrak{a}} X$ has an open neighbourhood $U$ over which, for every $N \geq 1$, there is an exact sequence of sheaves of modules of the form

$$
\left.\left.\left.\left.\mathcal{O}^{m_{N}}\right|_{U} \stackrel{\varphi_{N-1}}{\longrightarrow} \cdots \stackrel{\varphi_{2}}{\longrightarrow} \mathcal{O}^{m_{2}}\right|_{U} \stackrel{\varphi_{1}}{\longrightarrow} \mathcal{O}^{m_{1}}\right|_{U} \stackrel{\varphi_{0}}{\longrightarrow} \mathcal{A}\right|_{U} \longrightarrow 0,
$$

where $\varphi_{i}, 0 \leq i \leq N-1$, are analytic homomorphisms.

An analytic sheaf $\mathcal{A}$ on $c_{\mathfrak{a}} X$ is called a Fréchet sheaf if for each open set $U \in \mathfrak{B}$ the module of sections $\Gamma(U, \mathcal{A})$ of $\mathcal{A}$ over $U$ is endowed with topology of a Fréchet space.

Proposition 4.7. Every coherent sheaf can be turned in a unique way into a Fréchet sheaf so that the following conditions are satisfied:

(1) If $\mathcal{A}$ is a coherent subsheaf of $\mathcal{O}$, then for any open subset $U \in \mathfrak{B}$ the module of sections $\Gamma(U, \mathcal{A})$ has topology of uniform convergence on compact subsets of $U$.

(2) If $\mathcal{A}, \mathcal{B}$ are coherent sheaves on $c_{\mathfrak{a}} X$, then for any $U \in \mathfrak{B}$ the spaces $\Gamma(U, \mathcal{A})$, $\Gamma(U, \mathcal{B})$ are Fréchet spaces, and any analytic homomorphism $\varphi: \mathcal{A} \rightarrow \mathcal{B}$ is continuous in the sense that the homomorphisms of sections of $\mathcal{A}$ and $\mathcal{B}$ over sets $U \in \mathfrak{B}$ induced by $\varphi$ are continuous.

The topology on $\Gamma(U, \mathcal{A})$ can be defined by a family of semi-norms

$$
\|f\|_{V_{k}}:=\inf _{h}\left\{\sup _{x \in V_{k}}|h(x)|: h \in \Gamma\left(V_{k}, \mathcal{O}^{m_{1}}\right), f=\bar{\varphi}_{0}(h)\right\},
$$

where $\bar{\varphi}_{0}$ is the homomorphism of sections induced by $\varphi_{0}$ in (4.5), and open sets $V_{k} \in \mathfrak{B}$ are such that $V_{k} \Subset V_{k+1} \Subset U$ for all $k$, and $U=\cup_{k} V_{k}$ (see Lemma $5.3(2)$ below for existence of such an exhaustion of $U)$. 
The proof essentially repeats that of an analogous result for coherent analytic sheaves on complex manifolds, see, e.g., [24]. For the sake of completeness, we provide the proof of the proposition in the Appendix.

Theorem 4.8 (Runge-type approximation). Let $X_{0}$ be a Stein manifold and $\mathcal{A}$ be a coherent sheaf on $c_{\mathfrak{a}} X$. Suppose that $Y_{0} \Subset X_{0}, \hat{Y} \subset c_{\mathfrak{a}} X$ are open and such that

(1) either $Y_{0}$ is holomorphically convex in $X_{0}$ and $\hat{Y}=\bar{p}^{-1}\left(Y_{0}\right)$,

(2) or $Y_{0}$ is holomorphically convex in $X_{0}$ and is contained in a simply connected open subset of $X_{0}$, and $\hat{Y}=\hat{\Pi}\left(Y_{0}, K\right)$ for some $K \in \mathfrak{Q}$ (see subsection 4.3$)$.

Then the image of the restriction map $\Gamma\left(c_{\mathfrak{a}} X, \mathcal{A}\right) \rightarrow \Gamma(\hat{Y}, \mathcal{A})$ is dense (in the topology of Proposition 4.7).

\section{Proofs: preliminaries}

\section{1. Čech cohomology}

For a topological space $X$ and a sheaf of abelian groups $\mathcal{S}$ on $X$ let $\Gamma(X, \mathcal{S})$ denote the abelian group of sections of $\mathcal{S}$ over $X$.

Let $\mathcal{U}$ be an open cover of $X$. By $\mathcal{C}^{i}(\mathcal{U}, \mathcal{S})$ we denote the space of Čech $i$-cochains with values in $\mathcal{S}$, by $\delta: \mathcal{C}^{i}(\mathcal{U}, \mathcal{S}) \rightarrow \mathcal{C}^{i+1}(\mathcal{U}, \mathcal{R})$ the Cech coboundary operator (see, e.g., [20] for details), by $\mathcal{Z}^{i}(\mathcal{U}, \mathcal{S}):=\left\{\sigma \in \mathcal{C}^{i}(\mathcal{U}, \mathcal{S}): \delta \sigma=0\right\}$ the space of $i$-cocycles, and by $\mathcal{B}^{i}(\mathcal{U}, \mathcal{S}):=\left\{\sigma \in \mathcal{Z}^{i}(\mathcal{U}, \mathcal{S}): \sigma=\delta(\eta), \eta \in \mathcal{C}^{i-1}(\mathcal{U}, \mathcal{S})\right\}$ the space of $i$-coboundaries. The Cech cohomology groups $H^{i}(\mathcal{U}, S)(i \geq 0)$ of $\mathcal{U}$ with values in $\mathcal{S}$ are defined by

$$
H^{i}(\mathcal{U}, S):=\mathcal{Z}^{i}(\mathcal{U}, \mathcal{S}) / \mathcal{B}^{i}(\mathcal{U}, \mathcal{S}), \quad i \geq 1
$$

and $H^{0}(\mathcal{U}, \mathcal{S}):=\Gamma(\mathcal{U}, \mathcal{S})$.

\section{2. $\bar{\partial}$-equation}

Let $B$ be a complex Banach space, $D_{0} \subset X_{0}$ be a strictly pseudoconvex domain in a complex manifold $X_{0}$. We fix a system of local coordinates on $D_{0}$ and consider a cover $\left\{W_{0, i}\right\}_{i \geq 1}$ of $D_{0}$ by coordinate patches. By $\Lambda_{b}^{(0, q)}\left(D_{0}, B\right), q \geq 0$, we denote the space of bounded continuous $B$-valued $(0, q)$-forms $\omega$ on $D_{0}$ endowed with norm

$$
\|\omega\|_{D_{0}}=\|\omega\|_{D_{0}}^{(0, q)}:=\sup _{i, \alpha, x \in W_{0, i}}\left\|\omega_{\alpha, i}(x)\right\|_{B}
$$

where $\omega_{\alpha, i}\left(\alpha\right.$ is a multiindex) are coefficients of form $\left.\omega\right|_{W_{0, i}} \in \Lambda_{b}^{(0, q)}\left(W_{0, i}, B\right)$ written in the local coordinates on $W_{0, i}$.

The next lemma follows easily from results in [25] (proved for $B=\mathbb{C}$ ), as all integral presentations and estimates are preserved when passing to the case of Banach-valued forms. 
Lemma 5.1. There exists a bounded linear operator

$$
R_{D_{0}, B}: \Lambda_{b}^{(0, q)}\left(D_{0}, B\right) \rightarrow \Lambda_{b}^{(0, q-1)}\left(D_{0}, B\right), \quad q \geq 1
$$

such that, if $\omega \in \Lambda_{b}^{(0, q)}\left(D_{0}, B\right)$ is $C^{\infty}$ and satisfies $\bar{\partial} \omega=0$ on $D_{0}$, then $\bar{\partial} R_{D_{0}, B}(\omega)$ $=\omega$ on $D_{0}$.

\subsection{Auxiliary topological results}

In our proofs, we make use of the following results.

Let $\mathcal{L}=\left\{L_{i}\right\}$ be an open cover of $\hat{G}_{\mathfrak{a}}$. Recall that a refinement of $\mathcal{L}$ is an open cover $\mathcal{L}^{\prime}=\left\{L_{j}^{\prime}\right\}$ of $\hat{G}_{\mathfrak{a}}$ such that each $L_{j}^{\prime} \Subset L_{i}$ for some $i=i(j)$.

Since $\hat{G}_{\mathfrak{a}}$ is compact, each open cover of $\hat{G}_{\mathfrak{a}}$ has a finite subcover.

Lemma 5.2. Let $\mathcal{L}$ be a finite open cover of $\hat{G}_{\mathfrak{a}}$. There exist finite refinements $\mathcal{L}^{k}=\left\{L_{j}^{k}: L_{j}^{k} \in \mathfrak{Q}\right\}$ of $\mathcal{L}$ of the same cardinality such that $L_{j}^{k+1} \Subset L_{j}^{k}$ for all $j, k$.

Proof. Since $\hat{G}_{\mathfrak{a}}$ is compact, there exists a finite refinement $\mathcal{L}^{\prime}=\left\{L_{j}^{\prime}\right\}$ of $\mathcal{L}=\left\{L_{i}\right\}$ such that every $L_{j}^{\prime} \Subset L_{i}$ for some $i=i(j)$, and functions $\left\{\rho_{j}\right\} \subset C\left(\hat{G}_{\mathfrak{a}}\right)$ such that $\rho_{j} \equiv 1$ on $\bar{L}_{j}^{\prime}, \rho_{j} \equiv 0$ on $\hat{G}_{\mathfrak{a}} \backslash L_{i}$. We set $L_{j}^{k}:=\left\{\eta \in \hat{G}_{\mathfrak{a}}: \rho_{j}(\eta)>1-1 /(2 k)\right\}$, $k \geq 1$. By definition, $L_{j}^{k} \in \mathfrak{Q}$ for all $j, k$ (see (4.3)). It follows that $\mathcal{L}^{k}:=\left\{L_{j}^{k}\right\}$ are the required refinements of $\mathcal{L}$.

Lemma 5.3. Let $K \in \mathfrak{Q}, U_{0} \subset X_{0}$ be open. We set $U:=U_{0} \times K$. The following is true:

(1) There exist open subsets $N_{k} \in \mathfrak{Q}, 1 \leq k<\infty$, such that $N_{k} \Subset N_{k+1} \Subset K$ for all $k$ and $K=\cup_{k} N_{k}$.

(2) There are open subsets $V_{k}=V_{0, k} \times N_{k}, 1 \leq k<\infty$, such that $V_{k} \Subset V_{k+1} \Subset U$ for all $k$ and $U=\cup_{k} V_{k}$. Here $V_{0, k} \Subset U_{0}$ is open and $N_{k} \in \mathfrak{Q}$ for all $k$.

(3) Let $L \in \mathfrak{Q}$ be such that $L \Subset K$. There exists a collection of sets $L^{m} \in \mathfrak{Q}$, $m \geq 1$, such that $L \Subset \cdots \Subset L^{m+1} \Subset L^{m} \Subset \cdots \Subset L^{1} \Subset K$ for all $m$.

(4) Let $N \Subset K$ and $\left\{L_{i}\right\}$ be a finite collection of open subsets of $K$ such that $N \Subset \cup_{i} L_{i}$. There exists a finite family of open subsets $L_{j}^{\prime} \subset K, L_{j}^{\prime} \in \mathfrak{Q}$, such that $N \Subset \cup_{j} L_{j}^{\prime}$ and for each $j$ we have $L_{j}^{\prime} \Subset L_{i}$ for some $i=i(j)$.

Proof. (1) Recall that the basis $\mathfrak{Q}$ of topology of $\hat{G}_{\mathfrak{a}}$ consists of sublevel sets of functions in $C\left(\hat{G}_{\mathfrak{a}}\right)$, see $(4.3)$, so $\left.K=\left\{\eta \in \hat{G}_{\mathfrak{a}}: \max _{1 \leq i \leq m} \mid h_{i}(\eta)\right)-h_{i}\left(\eta_{0}\right) \mid<\varepsilon\right\}$ for some $\eta_{0} \in \hat{G}_{\mathfrak{a}}, h_{1}, \ldots, h_{m} \in C\left(\hat{G}_{\mathfrak{a}}\right)$ and $\varepsilon>0$. Let $\mathfrak{a}^{\prime}$ be the subalgebra of $C\left(\hat{G}_{\mathfrak{a}}\right)$ generated by functions $h_{1}, \ldots, h_{m}, \bar{h}_{1}, \ldots, \bar{h}_{m}$. Since algebra $\mathfrak{a}^{\prime}$ is finitely generated, the maximal ideal space $M_{\mathfrak{a}^{\prime}}$ of $\mathfrak{a}^{\prime}$ is a compact subset of some $\mathbb{C}^{p}$, and we have $\mathfrak{a}^{\prime} \cong C\left(M_{\mathfrak{a}^{\prime}}\right)$. The map $\pi: \hat{G}_{\mathfrak{a}} \rightarrow M_{\mathfrak{a}^{\prime}}$ adjoint to the inclusion $\mathfrak{a}^{\prime} \subset C\left(\hat{G}_{\mathfrak{a}}\right)$ is proper and surjective. By definition, there exists an open subset $K^{\prime} \subset M_{\mathfrak{a}^{\prime}}$ such that $K=\pi^{-1}\left(K^{\prime}\right)$. Since $M_{\mathfrak{a}^{\prime}}$ is a compact metric space (as a compact subset of $\mathbb{C}^{p}$ ), there exist open subsets $N_{k}^{\prime} \subset M_{\mathfrak{a}^{\prime}}$ such that $N_{k-1}^{\prime} \Subset N_{k}^{\prime} \Subset K^{\prime}$ for all $k$ and 
$K^{\prime}=\cup_{k} N_{k}^{\prime}$. We define $N_{k}:=\pi^{-1}\left(N_{k}\right) \in \mathfrak{Q}$. Clearly, each set $N_{k}^{\prime}$ can be chosen in the form $N_{k}^{\prime}=\left\{y \in M_{\mathfrak{a}^{\prime}}: \max _{1 \leq i \leq r_{k}}\left|f_{i k}(y)-f_{i k}\left(y_{0}\right)\right|<\varepsilon\right\}$ for some $y_{0} \in M_{\mathfrak{a}^{\prime}}$, $f_{i k} \in C\left(M_{\mathfrak{a}^{\prime}}\right)$ and $\varepsilon>0$. Since $\pi^{*} C\left(M_{\mathfrak{a}^{\prime}}\right) \subset C\left(\hat{G}_{\mathfrak{a}}\right), N_{k} \in \mathfrak{Q}$ (see (4.3)) as required.

A similar argument yields (3).

(2) It is clear that there exists a sequence of open sets $V_{0, k}$ such that $V_{0, k} \Subset$ $V_{0, k+1} \Subset U_{0}$ for all $k$ and $U_{0}=\cup_{k} V_{0, k}$. We set $V_{k}:=V_{0, k} \times N_{k}$.

(4) We apply Lemma 5.2 to the finite open cover of $\hat{G}_{\mathfrak{a}}$ consisting of the sets $L_{i}$ and set $\hat{G}_{\mathfrak{a}} \backslash \bar{N}$ to obtain a finite refinement $\left\{L_{j}^{\prime}\right\} \subset \mathfrak{Q}$ of this cover. We exclude subsets $L_{j}^{\prime}$ such that $L_{j}^{\prime} \Subset \hat{G}_{\mathfrak{a}} \backslash \bar{N}$. Then for the obtained family $\bar{N} \subset \cup_{j} L_{j}^{\prime}$, and by the definition of the refinement, for each $j$ we have $L_{j}^{\prime} \Subset L_{i}$ for some $i$, as required.

\section{Proof of Proposition 2.3}

We retain notation of the proposition. Given $f \in \mathcal{O}_{\mathfrak{a}}(X)$ denote $f_{x_{0}}:=\left.f\right|_{p^{-1}\left(x_{0}\right)}$. Let $\hat{f}_{x_{0}} \in C\left(\hat{G}_{\mathfrak{a}}\right)$ be such that $j^{*} \hat{f}_{x_{0}}=f_{x_{0}}$. The family $\left\{\hat{f}_{x_{0}}\right\}_{x_{0} \in X_{0}}$ determines a function $\hat{f}$ on $c_{\mathfrak{a}} X$ such that $\hat{f}(x)=\hat{f}_{x_{0}}(x)$ for $x_{0}:=\bar{p}(x)$. Using a normal family argument one shows that $\hat{f} \in \mathcal{O}\left(c_{\mathfrak{a}} X\right)$, see, e.g., [33] or [12], Lemma 2.3, for similar results. Clearly, $\iota^{*} \hat{f}=f$. Since the homomorphism ${ }^{\wedge}: \mathfrak{a} \rightarrow C\left(\hat{G}_{\mathfrak{a}}\right)$ is an injection, the constructed homomorphism $i: \mathcal{O}_{\mathfrak{a}}(X) \rightarrow \mathcal{O}\left(c_{\mathfrak{a}} X\right), i(f):=\hat{f}$, is an injection as well. This completes the proof of the first assertion.

For the proof of the second assertion suppose that $\mathfrak{a}$ is self-adjoint. Then $\mathfrak{a} \cong C\left(\hat{G}_{\mathfrak{a}}\right)$ and we can define the inverse homomorphism $i^{-1}: \mathcal{O}\left(c_{\mathfrak{a}} X\right) \rightarrow \mathcal{O}(X)$ by the formula

$$
i^{-1}(\hat{f}):=\iota^{*} \hat{f}, \quad \hat{f} \in \mathcal{O}\left(c_{\mathfrak{a}} X\right) .
$$

Since $\left.i^{-1}(\hat{f})\right|_{p^{-1}\left(x_{0}\right)}=j^{*}\left(\left.\hat{f}\right|_{\bar{p}^{-1}\left(x_{0}\right)}\right) \in \mathfrak{a}$ for all $x_{0} \in X_{0}$, we have $i^{-1}(\hat{f}) \in \mathcal{O}_{\mathfrak{a}}(X)$, i.e., $i^{-1}$ maps $\mathcal{O}\left(c_{\mathfrak{a}} X\right)$ into $\mathcal{O}_{\mathfrak{a}}(X)$.

\section{Proofs of Theorems 2.5, 2.6 and 4.8 and Proposition 4.7}

In what follows all polydisks are assumed to have finite polyradii.

Proof of Theorem 2.6. We will need the following results.

Proposition 7.1. Let $U:=\hat{\Pi}\left(U_{0}, K\right)$, where $U_{0} \subset X_{0}$ is open and biholomorphic to an open polydisk in $\mathbb{C}^{n}$, and $K \in \mathfrak{Q}$ (see (4.3)).

The following is true:

(1) Let $\mathcal{R}$ be an analytic sheaf over $U$ having a free resolution of length $4 N$ :

$$
\left.\left.\left.\left.\mathcal{O}^{k_{4 N}}\right|_{U} \stackrel{\varphi_{4 N-1}}{\longrightarrow} \cdots \stackrel{\varphi_{2}}{\longrightarrow} \mathcal{O}^{k_{2}}\right|_{U} \stackrel{\varphi_{1}}{\longrightarrow} \mathcal{O}^{k_{1}}\right|_{U} \stackrel{\varphi_{0}}{\longrightarrow} \mathcal{R}\right|_{W} \longrightarrow 0
$$


If $N \geq n:=\operatorname{dim}_{\mathbb{C}} U_{0}$, then the induced sequence of sections truncated to the $N$-th term

$$
\Gamma\left(U, \mathcal{O}^{k_{N}}\right) \stackrel{\bar{\varphi}_{N-1}}{\longrightarrow} \cdots \stackrel{\bar{\varphi}_{2}}{\longrightarrow} \Gamma\left(U, \mathcal{O}^{k_{2}}\right) \stackrel{\bar{\varphi}_{1}}{\longrightarrow} \Gamma\left(U, \mathcal{O}^{k_{1}}\right) \stackrel{\bar{\varphi}_{0}}{\longrightarrow} \Gamma(U, \mathcal{R}) \longrightarrow 0
$$

is exact.

(2) Suppose that free resolution (7.1) exists for every $N$. Then $H^{i}(U, \mathcal{R})=0$ for all $i \geq 1$.

Let $\mathcal{A}$ be a coherent sheaf on $c_{\mathfrak{a}} X$.

Proposition 7.2. Every point $x_{0} \in X_{0}$ has a neighbourhood $U_{0}$ such that for each $N \geq 1$ there exists a free resolution of sheaf $\mathcal{A}$ over $\bar{p}^{-1}\left(U_{0}\right)$ having length $N$ (see Definition 2.4).

(In other words, we may assume that the open sets $W$ in Definition 2.4 have the form $U=\bar{p}^{-1}\left(U_{0}\right), U_{0} \subset X_{0}$ is open.)

We prove Propositions 7.1 and 7.2 in subsections 7.1 and 7.2 , respectively.

Now, let $\hat{\mathcal{A}}:=\bar{p}_{*} \mathcal{A}$ be the direct image of sheaf $\mathcal{A}$ under projection $\bar{p}: c_{\mathfrak{a}} X \rightarrow X_{0}$. By definition, $\hat{\mathcal{A}}$ is a sheaf of modules over the sheaf of rings $\mathcal{O}^{C\left(\hat{G}_{\mathfrak{a}}\right)}$ of germs of holomorphic functions on $X_{0}$ taking values in the Banach space $C\left(\hat{G}_{\mathfrak{a}}\right)$. By Propositions 7.2 and $7.1(2)$ every $x_{0} \in X_{0}$ has a basis of neighbourhoods $U_{0}$ such that $H^{i}(U, \mathcal{A})=0, i \geq 1, U:=\bar{p}^{-1}\left(U_{0}\right)$. Therefore,

$$
H^{i}\left(c_{\mathfrak{a}} X, \mathcal{A}\right) \cong H^{i}\left(X_{0}, \hat{\mathcal{A}}\right), \quad i \geq 0
$$

(see, e.g., [23], Ch. F, Cor. 6, for the proof). By definition, we have

$$
\Gamma(U, \mathcal{A}) \cong \Gamma\left(U_{0}, \hat{\mathcal{A}}\right), \quad \Gamma(U, \mathcal{O}) \cong \Gamma\left(U_{0}, \mathcal{O}^{C\left(\hat{G}_{\mathfrak{a}}\right)}\right) .
$$

It follows from Proposition 7.2 and Proposition 7.1 (1) that for every $x_{0} \in X_{0}$ and each $N \geq 1$ there exist a neighbourhood $U_{0}$ of $x_{0}$ and an exact sequence of sections

$$
\Gamma\left(U_{0},\left(\mathcal{O}^{C\left(\hat{G}_{\mathfrak{a}}\right)}\right)^{k_{N}}\right) \longrightarrow \cdots \rightarrow \Gamma\left(U_{0},\left(\mathcal{O}^{C\left(\hat{G}_{\mathfrak{a}}\right)}\right)^{k_{1}}\right) \rightarrow \Gamma\left(U_{0}, \hat{\mathcal{A}}\right) \longrightarrow 0 .
$$

By definition, this means that we have an exact sequence of sheaves

$$
\left.\left.\left.\left(\mathcal{O}^{C\left(\hat{G}_{\mathfrak{a}}\right)}\right)^{k_{N}}\right|_{U_{0}} \longrightarrow \cdots \rightarrow\left(\mathcal{O}^{C\left(\hat{G}_{\mathfrak{a}}\right)}\right)^{k_{1}}\right|_{U_{0}} \longrightarrow \hat{\mathcal{A}}\right|_{U_{0}} \longrightarrow 0 .
$$

Next, for every open set $U_{0} \subset X_{0}$ the spaces of sections $\Gamma\left(U_{0}, \hat{\mathcal{A}}\right), \Gamma\left(U_{0}, \mathcal{O}^{C\left(\hat{G}_{\mathfrak{a}}\right)}\right)$ can be endowed with a Fréchet topology so that the homomorphisms of sections induced by sheaf homomorphisms in (7.3) are continuous; indeed, since $\Gamma\left(U_{0}, \hat{\mathcal{A}}\right) \cong$ $\Gamma(U, \mathcal{A}), \Gamma\left(U_{0}, \mathcal{O}^{C\left(\hat{G}_{\mathfrak{a}}\right)}\right) \cong \Gamma(U, \mathcal{O})$, this follows from Proposition 4.7 with $U=$ $\bar{p}^{-1}\left(U_{0}\right)$ (we prove Proposition 4.7 below, using the results that will be obtained in the proofs of Propositions 7.1 and 7.2). Hence, in the terminology of [30], $\hat{\mathcal{A}}$ is a Banach coherent analytic Fréchet sheaf. Therefore, according to Theorem 2.3 (iii) in [30], $H^{i}\left(X_{0}, \hat{\mathcal{A}}\right)=0$ for all $i \geq 1$. Isomorphism (7.2) now implies the required statement. The proof of Theorem 2.6 is complete. 
Proof of Theorem 4.8. We need to show that sections of a coherent sheaf $\mathcal{A}$ over certain subsets of $c_{\mathfrak{a}} X$ (we distinguish two kind of such subsets) can be approximated by the global sections of $\mathcal{A}$ over $c_{\mathfrak{a}} X$.

(1) Due to the arguments from the previous proof, we have isomorphisms of Fréchet spaces $\Gamma\left(c_{\mathfrak{a}} X, \mathcal{A}\right) \cong \Gamma\left(X_{0}, \hat{\mathcal{A}}\right), \Gamma(\hat{Y}, \mathcal{A}) \cong \Gamma\left(Y_{0}, \hat{\mathcal{A}}\right)$. Now, the result follows from Theorem 2.3 (iv) in [30] applied to $\hat{\mathcal{A}}$.

(2) It suffices to show that the restriction map $\Gamma\left(\bar{p}^{-1}\left(Y_{0}\right), \mathcal{A}\right) \rightarrow \Gamma(\hat{Y}, \mathcal{A})$ has dense image and then to apply the result of case (1).

We have $\hat{Y}=\hat{\Pi}\left(Y_{0}, K\right)$ for some $Y_{0} \Subset X_{0}$ open simply connected, and $K \in \mathfrak{Q}$. Since $\hat{Y} \in \mathfrak{B}$, we may use the last assertion of Proposition 4.7: it suffices to show that given a section $f \in \Gamma(\hat{Y}, \mathcal{A})$ for every $\varepsilon>0$ and every $k$ there exists a section $\tilde{f}_{k} \in \Gamma\left(\bar{p}^{-1}\left(Y_{0}\right), \mathcal{A}\right)$ such that $\left\|f-\tilde{f}_{k}\right\|_{V_{k}}<\varepsilon$.

Without loss of generality we may identify $\hat{Y}$ with $Y_{0} \times K$, and $\bar{p}^{-1}\left(Y_{0}\right)$ with $Y_{0} \times$ $\hat{G}_{\mathfrak{a}}$ (see subsection 4.2). Then sets $V_{k}$ have the form $V_{k}=V_{0, k} \times N_{k}$, where each $V_{0, k}$ is open and simply connected and $N_{k} \in \mathfrak{Q}$ are such that $N_{k} \Subset N_{k+1} \Subset K$ for all $k$, and $K=\cup_{k} N_{k}$ (see Lemma $5.3(1)$ ). Since space $\hat{G}_{\mathfrak{a}}$ is compact and, therefore, normal, for each $k$ there exists a function $\rho_{k} \in C\left(\hat{G}_{\mathfrak{a}}\right)$ such that $0 \leq \rho_{k} \leq 1$ on $\hat{G}_{\mathfrak{a}}$, $\rho_{k} \equiv 1$ on $N_{k}$, and $\rho_{k} \equiv 0$ on $\hat{G}_{\mathfrak{a}} \backslash \bar{N}_{k+1}$. Since $\Gamma\left(Y_{0} \times K, \mathcal{A}\right)$ is a module over $\Gamma\left(Y_{0} \times K, \mathcal{O}\right)$, we can define $\tilde{f}_{k}:=\rho_{k} f \in \Gamma\left(Y_{0} \times \hat{G}_{\mathfrak{a}}, \mathcal{A}\right)$. Then $f-\tilde{f}_{k}=0$ on $Y_{0} \times N_{k}$, so $\left\|f-\tilde{f}_{k}\right\|_{V_{k}}=0$. Thus, $\tilde{f}_{k}$ is the required approximation.

Proof of Theorem 2.5. Let $N \geq n$. Since sheaf $\mathcal{A}$ is coherent, there exists a neighbourhood $U$ of $x$ over which there is a free resolution

$$
\left.\left.\left.\left.\mathcal{O}^{m_{4 N}}\right|_{U} \stackrel{\varphi_{4 N-1}}{\longrightarrow} \cdots \stackrel{\varphi_{2}}{\longrightarrow} \mathcal{O}^{m_{2}}\right|_{U} \stackrel{\varphi_{1}}{\longrightarrow} \mathcal{O}^{m_{1}}\right|_{U} \stackrel{\varphi_{0}}{\longrightarrow} \mathcal{A}\right|_{U} \longrightarrow 0
$$

of length $4 N$. It follows from the exactness of sequence (7.4) that there exist sections $h_{1}, \ldots, h_{m_{1}} \in \Gamma(U, \mathcal{A})$ that generate ${ }_{x} \mathcal{A}$ as an ${ }_{x} \mathcal{O}$-module. Now, it suffices to show that there exist a neighbourhood $V \subset U$ of $x$, global sections $f_{1}, \ldots, f_{m_{1}} \in$ $\Gamma\left(c_{\mathfrak{a}} X, \mathcal{A}\right)$ and functions $r_{i j} \in \mathcal{O}(V), 1 \leq i, j \leq m_{1}$, such that

$$
\left.h_{i}\right|_{V}=\left.\sum_{j=1}^{m_{1}} r_{i j} f_{j}\right|_{V}, \quad 1 \leq i \leq m_{1} .
$$

Without loss of generality we may assume that $U=\hat{\Pi}\left(U_{0}, K\right) \in \mathfrak{B}$, where $U_{0} \subset X_{0}$ is biholomorphic to an open polydisk in $\mathbb{C}^{n}$ and is holomorphically convex in $X_{0}$, and $K \in \mathfrak{Q}$. By Proposition 4.7 the topology on $\Gamma(W, \mathcal{A})$ is determined by seminorms

$$
\|h\|_{V_{k}}:=\inf _{h}\left\{\sup _{x \in V_{k}}|g(x)|: g \in \Gamma\left(V_{k}, \mathcal{O}^{m_{1}}\right), h=\bar{\varphi}_{0}(g)\right\},
$$

where $\bar{\varphi}_{0}$ is the homomorphism of sections induced by $\varphi_{0}$ in (7.4), and open sets $V_{k} \in \mathfrak{B}$ are such that $V_{k} \Subset V_{k+1} \Subset W$ for all $k$, and $W=\cup_{k} V_{k}$, see Lemma $5.3(2)$; by definition, $V_{k}=V_{0, k} \times N_{k}$, where $V_{0, k} \Subset U_{0}, N_{k} \Subset K$ are open. Without loss of generality we may assume that each $V_{0, k}$ is biholomorphic to an open polydisk in $\mathbb{C}^{n}$ and is holomorphically convex in $X_{0}$. 
Let $V:=V_{k_{0}}$, where $k_{0}$ is chosen so that $x \in V_{k_{0}}$. It follows from the proof of Theorem 4.8 (case $(2)$ for $\hat{Y}:=U$ ) that for every $\varepsilon>0$ there exist sections $f_{1}, \ldots, f_{m_{1}} \in \Gamma\left(c_{\mathfrak{a}} X, \mathcal{A}\right)$ such that $\left\|h_{i}-f_{i}\right\|_{V}<\varepsilon$ for all $i$. Now, by Proposition $7.1(1)$ the sequence of sections corresponding to (7.4),

$$
\cdots \longrightarrow \Gamma\left(V, \mathcal{O}^{m_{1}}\right) \stackrel{\bar{\varphi}_{0}}{\longrightarrow} \Gamma(V, \mathcal{A}) \longrightarrow 0
$$

is exact. Note that $\Gamma\left(V, \mathcal{O}^{m_{1}}\right)$ consists of $m_{1}$-tuples of holomorphic functions on $V$. Let $\tilde{h}_{i}:=(0, \ldots, 1, \ldots, 0)$ (1 is in the $i$-th position), $1 \leq i \leq m_{1}$. Without loss of generality we may assume that $\left.h_{i}\right|_{V}=\bar{\varphi}_{0}\left(\tilde{h}_{i}\right)$. Since $\bar{\varphi}_{0}$ is surjective, there exist functions $\tilde{f}_{i} \in \Gamma\left(V, \mathcal{O}^{m_{1}}\right)$ such that $\bar{\varphi}_{0}\left(\tilde{f}_{i}\right)=\left.f_{i}\right|_{V}$. It follows from the definition of semi-norm $\|\cdot\|_{V}$, see (7.6), that functions $\tilde{f}_{i}$ can be chosen in such a way that

$$
\sup _{x \in V}\left|\tilde{h}_{i}(x)-\tilde{f}_{i}(x)\right|<2 \varepsilon .
$$

Since $\bar{\varphi}_{0}$ is a $\mathcal{O}(V)$-module homomorphism, the required identity (7.5) would follow once we found functions $r_{i j} \in \Gamma(V, \mathcal{O}), 1 \leq i, j \leq m_{1}$, such that

$$
\tilde{h}_{i}=\sum_{j=1}^{m_{1}} r_{i j} \tilde{f}_{j}, \quad 1 \leq i \leq m_{1} .
$$

The latter system of linear equations (with respect to $r_{i j}$ ) can be rewritten as a matrix equation $H=F R$ with respect to $R=\left(r_{i j}\right)_{i, j=1}^{m_{1}} \in \mathcal{O}\left(V, M_{n}(\mathbb{C})\right)$, where $M_{n}(\mathbb{C})$ denotes the set of $n \times n$ complex matrices, $H=\left(\tilde{h}_{i}\right)_{i=1}^{m_{1}} \in \mathcal{O}\left(V, G L_{n}(\mathbb{C})\right)$ $\left(\tilde{h}_{i}\right.$ are the columns of $\left.H\right)$ is the identity matrix, here $G L_{n}(\mathbb{C}) \subset M_{n}(\mathbb{C})$ is the group of invertible matrices and $F=\left(\tilde{f}_{i}\right)_{i=1}^{m_{1}} \in \mathcal{O}\left(V, M_{n}(\mathbb{C})\right)\left(\tilde{f}_{i}\right.$ are the columns of $F$ ). Since $\varepsilon>0$ can be chosen arbitrarily small, in view of (7.8) we may assume that $F \in \mathcal{O}\left(V, G L_{n}(\mathbb{C})\right)$. Hence, we can define $R:=F^{-1} H$.

This completes the proof of Theorem 2.5.

\subsection{Proof of Proposition 7.1}

The (rather technical) proof of this proposition is presented at the end of this subsection. In the proof we will use the following preliminary results.

Let $U_{0} \Subset \mathbb{C}^{n}$ be an open polydisk, $K \in \mathfrak{Q}$ (see (4.3)). We set

$$
U:=U_{0} \times K
$$

The sets $U$ and $\hat{\Pi}\left(U_{0}, K\right) \subset c_{\mathfrak{a}} X$ are biholomorphic (see subsection 4.2). Definitions of an analytic homomorphism and a free resolution (of an analytic sheaf over an open subset of $c_{\mathfrak{a}} X$, see subsection 4.4) are transferred naturally to analytic sheaves over $U$. Thus, it suffices to prove Proposition 7.1 in the assumption that analytic sheaf $\mathcal{R}$ and free resolution (7.1) are given over $U$.

A function $f \in C(U)$ is said to be $C^{\infty}$ if all its derivatives with respect to variable $x \in U_{0}$ (in some local coordinates on $U_{0}$ ) are in $C(U)$. The algebra of $C^{\infty}$ functions on $U$ will be denoted by $C^{\infty}(U)$. 
Let $\Lambda^{p, q}\left(U_{0}\right)$ be the collection of all $C^{\infty}(p, q)$-forms on $U_{0}$. We define the space $\Lambda^{p, q}(U)$ of $C^{\infty}(p, q)$-forms on $U$ by the formula $\Lambda^{p, q}(U):=C^{\infty}(U) \otimes \Lambda^{p, q}\left(U_{0}\right)$, where $\otimes$ denotes symmetric tensor product.

We have an operator $\bar{\partial}: \Lambda^{p, q}(U) \rightarrow \Lambda^{p, q+1}(U)$ defined as follows. Suppose that $\omega \in \Lambda^{p, q}(U)$ is given (in local coordinates on $U_{0}$ ) by the formula

$$
\omega=\sum_{|I|=p} \sum_{|J|=q} f_{I J} d z_{I} \wedge d \bar{z}_{J}, \quad f_{I J} \in C^{\infty}(U),
$$

where $I=\left(i_{1}, \ldots, i_{p}\right), J=\left(j_{1}, \ldots, j_{q}\right), d z_{I}=d z_{i_{1}} \wedge \cdots \wedge d z_{i_{p}}, d \bar{z}_{J}=d \bar{z}_{j_{1}} \wedge \cdots \wedge d \bar{z}_{j_{q}}$ then

$$
\bar{\partial} f:=\sum_{|I|=p} \sum_{|J|=q} \bar{\partial} f_{I J} \wedge d z_{I} \wedge d \bar{z}_{J}
$$

where

$$
\bar{\partial} f_{I J}(z, \eta):=\sum_{j=1}^{n} \frac{\partial f_{I J}(z, \xi)}{\partial \bar{z}_{j}} d \bar{z}_{j}, \quad z=\left(z_{1}, \ldots, z_{n}\right), \quad(z, \xi) \in U=U_{0} \times K .
$$

A form $\omega \in \Lambda^{p, q}(U)$ is called $\bar{\partial}$-closed if $\bar{\partial} \omega=0$.

Let $\Lambda^{p, q}$ be the sheaf of germs of $C^{\infty}(p, q)$-forms on $U$, and $Z^{p, q} \subset \Lambda^{p, q}$ be the subsheaf of germs of $\bar{\partial}$-closed $(p, q)$-forms. Note that $Z^{0,0}=\mathcal{O}$.

In what follows we fix an open polydisk

$$
V_{0} \Subset U_{0} .
$$

Let $W_{0} \subset \bar{V}_{0}$ be open in $\bar{V}_{0}$ and such that $W_{0}=\bar{V}_{0} \cap \tilde{W}_{0}$ for some product domain $\tilde{W}_{0}=\tilde{W}_{0}^{1} \times \cdots \times \tilde{W}_{0}^{n} \Subset U_{0}$, where each $\tilde{W}_{0}^{i} \Subset \mathbb{C}(1 \leq i \leq n)$ is simply connected and has smooth boundary (clearly, given an open neighbourhood of $\bar{W}_{0}$ in $U_{0}$, we can find such a set $\tilde{W}_{0}$ contained in this neighbourhood).

Fix a subset $W_{0}^{\prime} \Subset W_{0}$ open in $\bar{V}_{0}$ and satisfying the same intersection condition as $W_{0}$ (i.e. $W_{0}^{\prime}$ coincides with the intersection of $\bar{V}_{0}$ and a product domain having the properties as described above). Let

$$
S \subset K \text { be a closed subset, and let } L^{\prime} \Subset L \subset S \text { be open in } S \text {. }
$$

Lemma 7.3. For every $\omega \in \Gamma\left(W_{0} \times L, Z^{0, q}\right)$ there exists $\eta \in \Gamma\left(\bar{W}_{0}^{\prime} \times \bar{L}^{\prime}, \Lambda^{0, q-1}\right)$ such that $\bar{\partial} \eta=\omega$.

Proof. By definition, a section of sheaf $Z^{0, q}$ over $W_{0} \times L$ is the restriction of a section of $Z^{0, q}$ over some open neighbourhood of $W_{0} \times L$. Therefore, we may assume, without loss of generality, that $L$ is open in $K$, and $\omega \in \Gamma\left(\tilde{W}_{0} \times L, Z^{0, q}\right)$ for some product domain $\tilde{W}_{0}$ as above.

Clearly, there exists a product domain $\hat{W}_{0} \Subset \tilde{W}_{0}$ open in $U_{0}$, where $\hat{W}_{0}=$ $\hat{W}_{0}^{1} \times \cdots \times \hat{W}_{0}^{n}$ and each domain $\hat{W}_{0}^{i} \Subset \tilde{W}_{0}^{i}$ has smooth boundary, such that $W_{0}^{\prime} \Subset \hat{W}_{0}$. Further, since $\hat{G}_{\mathfrak{a}}$ is a normal space, there exists an open set $L^{\prime \prime} \Subset L$ such that $L^{\prime} \Subset L^{\prime \prime}$. 
Let $C\left(\bar{L}^{\prime \prime}\right)$ be the Banach space of continuous functions on $\bar{L}^{\prime \prime}$ endowed with sup-norm, $\Lambda^{0, q}\left(\tilde{W}_{0}, C\left(\bar{L}^{\prime \prime}\right)\right)$ be the space of $C^{\infty} C\left(\bar{L}^{\prime \prime}\right)$-valued $(0, q)$-forms on $\tilde{W}_{0}$, and

$$
Z^{0, q}\left(\hat{W}_{0}, C\left(\bar{L}^{\prime \prime}\right)\right) \subset \Lambda^{0, q}\left(\hat{W}_{0}, C\left(\bar{L}^{\prime \prime}\right)\right)
$$

be the subspace of $\bar{\partial}_{C\left(\bar{L}^{\prime \prime}\right)}$-closed forms on $\hat{W}_{0}$. Here

$$
\bar{\partial}_{C\left(\bar{L}^{\prime \prime}\right)}: \Lambda^{0, q}\left(\hat{W}_{0}, C\left(\bar{L}^{\prime \prime}\right)\right) \rightarrow Z^{0, q+1}\left(\hat{W}_{0}, C\left(\bar{L}^{\prime \prime}\right)\right)
$$

is the standard operator of differentiation of $C\left(\bar{L}^{\prime \prime}\right)$-valued forms.

It is easy to see that the restriction to $\tilde{W}_{0} \times \bar{L}^{\prime \prime}$ of a form in $\Gamma\left(\tilde{W}_{0} \times L, \Lambda^{0, q}\right)$ can be naturally identified with a form in $\Lambda^{0, q}\left(\tilde{W}_{0}, C\left(\bar{L}^{\prime \prime}\right)\right)$ and, since $\hat{W}_{0} \times L^{\prime \prime}$ is a neighbourhood of $\bar{W}_{0}^{\prime} \times \bar{L}^{\prime}$, every form in $\Lambda^{0, q}\left(\tilde{W}_{0}, C\left(\bar{L}^{\prime \prime}\right)\right)$ determines (under such identification) a unique form in $\Gamma\left(\bar{W}_{0}^{\prime} \times \bar{L}^{\prime}, \Lambda^{0, q}\right)$; these identification maps commute with the actions of operators $\bar{\partial}$ and $\bar{\partial}_{C\left(\bar{L}^{\prime \prime}\right)}$. In particular, form $\omega$ determines a form $\hat{\omega} \in Z^{0, q}\left(\tilde{W}_{0}, C\left(\bar{L}^{\prime \prime}\right)\right)$. Note that since $\tilde{W}_{0} \Subset \mathbb{C}^{n}$ is a product domain, it is pseudoconvex. Hence $W_{0}$ admits an exhaustion by strictly pseudoconvex subdomains (see, e.g., [28]). Therefore, there exists a strictly pseudoconvex domain $D_{0} \Subset \tilde{W}_{0}$ such that $\hat{W}_{0} \Subset D_{0}$. We restrict form $\hat{\omega}$ to $D_{0}$ (clearly, $\left.\hat{\omega}\right|_{D_{0}}$ is bounded) and apply Lemma 5.1 , where we take $B:=C\left(\bar{L}^{\prime \prime}\right)$. We obtain that there exists a form $\hat{\eta} \in \Lambda^{0, q-1}\left(\hat{W}_{0}, C\left(\bar{L}^{\prime \prime}\right)\right)$ such that $\bar{\partial}_{C\left(\bar{L}^{\prime \prime}\right)} \hat{\eta}=\hat{\omega}$ over $\hat{W}_{0}$. It follows that the form $\eta \in \Gamma\left(\bar{W}_{0}^{\prime} \times \bar{L}^{\prime}, \Lambda^{0, q-1}\right)$ determined by $\hat{\eta}$ is the required one.

We will need the following:

Definition 7.4. We say that a finite open cover $\mathcal{U}=\left\{U_{\alpha}\right\}$ of $\bar{V}_{0} \times S$ (see (7.11) and $(7.12))$ is of class $(P)$ if the following conditions are satisfied:

(1) $U_{\alpha}=U_{0, l} \times L_{j}, \alpha=(l, j)$, where $\left\{U_{0, l}\right\}$ and $\left\{L_{j}\right\}$ are finite open covers of $\bar{V}_{0}$ and $S$, respectively;

(2) Each $L_{j}=S \cap \tilde{L}_{j}$ for some $\tilde{L}_{j} \in \mathfrak{Q}$ such that $\tilde{L}_{j} \subset K$;

(3) Each $U_{0, l}=\bar{V}_{0} \cap \tilde{U}_{0, l}$ for some product domain $\tilde{U}_{0, l}=\tilde{U}_{0, l}^{1} \times \cdots \times \tilde{U}_{0, l}^{n} \Subset U_{0}$, where domains $\tilde{U}_{0, l}^{i} \Subset \mathbb{C}(1 \leq i \leq n)$ are simply connected and has smooth boundaries.

Lemma 7.5. (1) Each open cover of $\bar{V}_{0} \times S$ has a refinement of class $(P)$.

(2) Each open cover of $\bar{V}_{0} \times S$ of class $(P)$ has a refinement of class $(P)$ of the same cardinality.

Proof. (1) Since $\bar{V}_{0}, S$ are compact, and hence their direct product $\bar{V}_{0} \times S$ is compact as well, there exists a refinement of a given open cover of $\bar{V}_{0} \times S$ by open sets of the form $U_{0, l} \times O_{j}$, where $\left\{U_{0, l}\right\}$ and $\left\{O_{i}\right\}$ are finite open covers of $\bar{V}_{0}$ and $S$, respectively. By the definition of the induced topology on $S$, there exist open sets $\tilde{O}_{i} \subset K$ such that $O_{i}=S \cap \tilde{O}_{i}$. Now, we apply Lemma $5.3(4)$ to $\left\{\tilde{O}_{i}\right\}$ (there we take $\bar{N}:=S)$ to obtain open sets $\left\{\tilde{L}_{j}\right\}$ such that $L_{j} \Subset L_{i}$ for some $i=i(j)$ and $\tilde{L}_{j} \in \mathfrak{Q}$ for all $j$. Finally, we set $L_{j}:=S \cap \tilde{L}_{j}$. The sets $U_{0, l} \times L_{j}$ form the required refinement of class $(P)$.

(2) Follows from assertions (3) and (4) of Lemma 5.3. 
Let $\mathcal{U}=\left\{U_{\alpha}:=U_{0, l} \times L_{j}\right\}$ be a finite open cover of $\bar{V}_{0} \times S$ of class $(P)$, and $\mathcal{U}^{\prime}=\left\{U_{\alpha}^{\prime}:=U_{0, l}^{\prime} \times L_{j}^{\prime}\right\}$ be a refinement of $\mathcal{U}$ of class $(P)$ of the same cardinality (see Lemma $7.5(2)$ ). By definition, $\left\{U_{0, l}^{\prime}\right\}$ and $\left\{L_{j}^{\prime}\right\}$ are refinements of open covers $\left\{U_{0, l}\right\}$ and $\left\{L_{j}\right\}$, respectively.

We have an injective refinement map $\mathfrak{U}_{\mathcal{U}, \mathcal{U}^{\prime}}: \mathcal{Z}^{i}(\mathcal{U}, \mathcal{R}) \rightarrow \mathcal{Z}^{i}\left(\mathcal{U}^{\prime}, \mathcal{R}\right)$ (see subsection 5.1 for notation). If no confusion arises, we write $\sigma$ for $\mathcal{U}_{\mathcal{U}} \mathcal{U}^{\prime}(\sigma)$.

Lemma 7.6. The following is true:

(1) Let $\sigma \in \mathcal{Z}^{i}(\mathcal{U}, \mathcal{O}), i \geq 1$. Then $\sigma \in \mathcal{B}^{i}\left(\mathcal{U}^{\prime}, \mathcal{O}\right)$.

(2) $H^{i}\left(\bar{V}_{0} \times S, \mathcal{O}\right)=0, i \geq 1$.

Proof. (1) We will prove a more general result: if $\sigma \in \mathcal{Z}^{i}\left(\mathcal{U}, Z^{0, q}\right), i \geq 1, q \geq 0$, then $\sigma \in \mathcal{B}^{i}\left(\mathcal{U}^{\prime}, Z^{0, q}\right)$. In particular, taking $q=0$ we obtain assertion (1).

Let $i=1, \sigma_{1} \in \mathcal{Z}^{1}\left(\mathcal{U}, Z^{0, q}\right)$. Since $\bar{V}_{0} \times S$ is a paracompact space, there exist partitions of unity $\left\{\lambda_{l}\right\}$ and $\left\{\rho_{j}\right\}$ subordinate to covers $\left\{U_{0, l}^{\prime}\right\}$ and $\left\{L_{j}^{\prime}\right\}\left(C^{\infty}\right.$ and continuous, respectively). We define a 0-cocycle $\sigma_{0}^{\infty} \in C^{0}\left(\mathcal{U}^{\prime}, \Lambda^{0, q}\right)$ by the formula

$$
\left(\sigma_{0}^{\infty}\right)_{\alpha}(x, \xi):=\sum_{\beta=(l, j)} \rho_{j}(\xi) \lambda_{l}(x)\left(\sigma_{1}\right)_{\beta, \alpha}(x, \xi), \quad(x, \xi) \in U_{\alpha}^{\prime} \quad \text { for all } \alpha .
$$

Since $\left(\sigma_{1}\right)_{\alpha, \beta}=\left(\delta \sigma_{0}^{\infty}\right)_{\alpha, \beta}=\left(\sigma_{0}^{\infty}\right)_{\alpha}-\left(\sigma_{0}^{\infty}\right)_{\beta}$ and $\bar{\partial}\left(\sigma_{1}\right)_{\alpha, \beta}=0$, the family $\left\{\bar{\partial}\left(\sigma_{0}^{\infty}\right)_{\alpha}\right\}$ determines $\omega \in \Gamma\left(\bar{V}_{0} \times S, Z^{0, q+1}\right),\left.\omega\right|_{U_{\alpha}}:=\bar{\partial}\left(\sigma_{0}^{\infty}\right)_{\alpha}$. By Lemma 7.3 (with $W_{0}^{\prime}=$ $W_{0}=\bar{V}_{0}$ and $\left.L^{\prime}=L=S\right)$ there exists $\eta \in \Gamma\left(\bar{V}_{0} \times S, \Lambda^{0, q}\right)$ such that $\bar{\partial} \eta=\omega$. We define a 0 -cochain $\sigma_{0} \in \mathcal{C}^{0}\left(\mathcal{U}^{\prime}, Z^{0, q}\right)$ by the formula $\left(\sigma_{0}\right)_{\alpha}=\left(\sigma_{0}^{\infty}\right)_{\alpha}-\eta$. It follows that $\sigma_{1}=\delta \sigma_{0}$; therefore $\sigma_{1} \in \mathcal{B}^{1}\left(\mathcal{U}^{\prime}, Z^{0, q}\right)$.

Using Lemma $7.5(2)$ we may assume that there exists a refinement $\mathcal{U}^{\prime \prime}=$ $\left\{U_{\alpha}^{\prime \prime}:=U_{0, l}^{\prime \prime} \times L_{j}^{\prime \prime}\right\}$ of cover $\mathcal{U}$ of class $(P)$ of the same cardinality as $\mathcal{U}$ such that $\mathcal{U}^{\prime}$ is a refinement of $\mathcal{U}^{\prime \prime}$.

Now, let $i>1$. Assume that we have shown for all $1 \leq l<i, q \geq 0$ that each $\sigma \in \mathcal{Z}^{l}\left(\mathcal{U}, Z^{0, q}\right)$ belongs to $\mathcal{B}^{l}\left(\mathcal{U}^{\prime \prime}, Z^{0, q}\right)$. For a given $\sigma_{i} \in \mathcal{Z}^{i}\left(\mathcal{U}, Z^{0, q}\right)$ we define an $(i-1)$-cocycle $\sigma_{i-1}^{\infty} \in \mathcal{C}^{i-1}\left(\mathcal{U}^{\prime \prime}, \Lambda^{0, q}\right)$ by the formula

$$
\left(\sigma_{i-1}^{\infty}\right)_{\alpha_{1}, \ldots, \alpha_{i}}(x, \xi):=\sum_{\beta=(l, j)} \rho_{j}(\xi) \lambda_{l}(x)\left(\sigma_{i}\right)_{\beta, \alpha_{1}, \ldots, \alpha_{i}}(x, \xi), \quad(x, \xi) \in U_{\alpha_{1}, \ldots, \alpha_{i}}^{\prime \prime}
$$

for all $\alpha_{1}, \ldots, \alpha_{i}$, where $U_{\alpha_{1}, \ldots, \alpha_{i}}^{\prime \prime}:=\cap_{r=1}^{i} U_{\alpha_{r}}^{\prime \prime} \neq \varnothing$.

We have $\delta\left(\sigma_{i-1}^{\infty}\right)=\sigma_{i}$, so $\bar{\partial} \delta\left(\sigma_{i-1}^{\infty}\right)=\delta\left(\bar{\partial} \sigma_{i-1}^{\infty}\right)=0$. Define $\mu_{i-1}:=\bar{\partial} \sigma_{i-1}^{\infty} \in$ $\mathcal{C}^{i-1}\left(\mathcal{U}^{\prime \prime}, Z^{0, q+1}\right)$. Since $\delta\left(\mu_{i-1}\right)=\bar{\partial} \mu_{i-1}=0$, by the induction assumption there exists an $(i-2)$-cochain $\mu_{i-2} \in \mathcal{C}^{i-2}\left(\mathcal{U}^{\prime \prime}, Z^{0, q}\right)$ such that $\delta\left(\mu_{i-2}\right)=\mu_{i-1}$ and $\bar{\partial} \mu_{i-2}=0$. Now, by Lemma $7.3(1)$ there exists an $(i-2)$-cochain $\eta_{i-2} \in$ $\mathcal{C}^{i-2}\left(\mathcal{U}^{\prime}, \Lambda^{0, q}\right)$ such that $\bar{\partial} \eta_{i-2}=\mu_{i-2}$. We define $\sigma_{i-1}:=\sigma_{i-1}^{\infty}-\delta\left(\eta_{i-2}\right)$. Then $\delta\left(\sigma_{i-1}\right)=\sigma_{i}$; so $\sigma_{i} \in \mathcal{B}^{i}\left(\mathcal{U}^{\prime}, Z^{0, q}\right)$, as required.

(2) By Lemma $7.5(1)$ any open cover of $\bar{V}_{0} \times S$ has a finite refinement of class $(P)$, hence the required result follows from (1). 
Let $\left\{V_{k}\right\}_{k=1}^{\infty}$ be the exhaustion of $U$ by open sets obtained in Lemma $5.3(2)$. By definition, each $V_{k}$ has the form $V_{k}=V_{0, k} \times N_{k}$, where $V_{0, k} \Subset U_{0}, N_{k} \Subset K$ are open, and $N_{k} \in \mathfrak{Q}$ for all $k$. Since $U_{0}$ is an open polydisk in $\mathbb{C}^{n}$, we may choose each $V_{0, k}$ to be an a open polydisk as well.

Definition 7.7 (see [20], Chapter IV). We say that an analytic sheaf $\mathcal{R}$ on $U$ satisfies the Runge condition if the following holds for every $k \geq 1$ :

(a) The space of sections $\Gamma\left(\bar{V}_{k}, \mathcal{R}\right)$ is endowed with a semi-norm $|\cdot|_{k}$ such that $\left.\Gamma(U, \mathcal{R})\right|_{\bar{V}_{k}}$ is dense in $\Gamma\left(\bar{V}_{k}, \mathcal{R}\right)$.

(b) There exist constants $M_{k}>0$ such that for every $f \in \Gamma\left(\bar{V}_{k+1}, \mathcal{R}\right)$ we have $\left.|f|_{\bar{V}_{k}}\right|_{k} \leq M_{k}|f|_{k+1}$.

(c) If $\left\{f_{j}\right\}$ is a Cauchy sequence in $\Gamma\left(\bar{V}_{k+1}, \mathcal{R}\right)$, then $\left\{\left.f_{j}\right|_{\bar{V}_{k}}\right\}$ has a limit in $\Gamma\left(\bar{V}_{k}, \mathcal{R}\right)$.

(d) If $f \in \Gamma\left(\bar{V}_{k+1}, \mathcal{R}\right)$ and $|f|_{k+1}=0$, then $\left.f\right|_{\bar{V}_{k}}=0$.

Lemma 7.8 (see [20], Chapter IV, for the proof). Let $\mathcal{R}$ be an analytic sheaf on $U$ The following is true:

(1) Suppose that $H^{i}\left(\bar{V}_{k}, \mathcal{R}\right)=0$ for all $i \geq 1, k \geq 1$. Then $H^{i}(U, \mathcal{R})=0$ for all $i \geq 2$.

(2) If $\mathcal{R}$ satisfies the Runge condition and $H^{1}\left(\bar{V}_{k}, \mathcal{R}\right)=0$ for all $k \geq 1$, then $H^{1}(U, \mathcal{R})=0$.

Lemma 7.9. The sheaf $\left.\mathcal{O}\right|_{U}$ satisfies the Runge condition.

Proof. For a given section $f \in \Gamma\left(\bar{V}_{k}, \mathcal{O}\right)$ let us denote by $\hat{f}(\omega) \in \mathbb{C}$ the common value of representatives of germ $f(\omega)$ at point $\omega \in \bar{V}_{k}$.

We endow each space $\Gamma\left(\bar{V}_{k}, \mathcal{O}\right)$ with semi-norm $|f|_{k}:=\sup _{\omega \in \bar{V}_{k}}|\hat{f}(\omega)|$. Conditions (b)-(d) are trivially satisfied. For the proof of (a), let us fix a section $f \in \Gamma\left(\bar{V}_{k}, \mathcal{O}\right)$. By definition, a section of sheaf $\mathcal{O}$ over $\bar{V}_{k}:=\bar{V}_{0, k} \times \bar{N}_{k}$ is the restriction of a section of $\mathcal{O}$ over an open neighbourhood of $\bar{V}_{k}$. In particular, there exists an open neighbourhood $L \subset K$ of $\bar{N}_{k}$ such that section $\left.f\right|_{\bar{V}_{k}}$ admits a bounded extension to $\bar{V}_{0, k} \times L$. Since $\hat{G}_{\mathfrak{a}}(\supset K)$ is a normal space, there exists a function $\rho_{k} \in C(K)$ such that $\rho_{k} \equiv 1$ on $\bar{N}_{k}$ and $\rho_{k} \equiv 0$ on $K \backslash L$. We set $\tilde{f}:=f \rho_{k} \in \Gamma\left(V_{0, k} \times K, \mathcal{O}\right)$. Then function $\tilde{f}$ determines a holomorphic function $\hat{f}$ defined in a neighbourhood of $\bar{V}_{0, k}$ with values in the Banach space $C_{b}(K)$ of bounded continuous functions on $K$ endowed with sup-norm $\|\cdot\|$. Now, we apply the Runge-type approximation theorem for Banach-valued holomorphic functions, see [15], to obtain that for every $\varepsilon>0$ there is a function $\hat{F} \in \mathcal{O}\left(U_{0}, C_{b}(K)\right)$ such that $\sup _{x \in \bar{V}_{0, k}}\|\hat{f}(x)-\hat{F}(x)\|<\varepsilon$. Then $\hat{F}$ determines a function $F \in \mathcal{O}(U)$ such that $\sup _{\omega \in \bar{V}_{k}}|f(\omega)-F(\omega)|<\varepsilon$, which implies (a).

Corollary 7.10. $H^{i}(U, \mathcal{O})=0$ for all $i \geq 1$.

Proof. Follows from Lemmas $7.6(2), 7.8$ and 7.9 . 
Lemma 7.11. Let $\mathcal{B}, \mathcal{R}$ be analytic sheaves on $U$. Let $V_{0} \Subset U_{0}$ be an open polydisk, $S \subset K$ a closed subset. Suppose that the sequence

$$
\mathcal{B} \stackrel{\psi}{\longrightarrow} \mathcal{R} \longrightarrow 0
$$

is exact. Then the sequence

$$
q_{*}\left(\left.\mathcal{B}\right|_{\bar{V}_{0} \times S}\right) \stackrel{q_{*} \psi}{\longrightarrow} q_{*}\left(\left.\mathcal{R}\right|_{\bar{V}_{0} \times S}\right) \longrightarrow 0
$$

is also exact. Here $q: \bar{V}_{0} \times S \rightarrow \bar{V}_{0}$ is the projection onto the first component and $q_{*}$ is the direct image functor.

Proof. We denote $\hat{\mathcal{B}}:=q_{*}\left(\left.\mathcal{B}\right|_{\bar{V}_{0} \times S}\right), \hat{\mathcal{R}}:=q_{*}\left(\left.\mathcal{R}\right|_{\bar{V}_{0} \times S}\right), \hat{\psi}:=q_{*} \psi$. We have to show that $\hat{\psi}$ is surjective. Given open subsets $W_{0} \subset \bar{V}_{0}, L \subset S$ by $\Psi_{W_{0} \times L}$ we denote the homomorphism of modules of sections $\Gamma\left(W_{0} \times L, \mathcal{B}\right) \rightarrow \Gamma\left(W_{0} \times L, \mathcal{R}\right)$ induced by $\psi$, and by $\hat{\Psi}_{W_{0}}$ the homomorphism of modules of sections $\Gamma\left(W_{0}, \hat{\mathcal{B}}\right) \rightarrow \Gamma\left(W_{0}, \hat{\mathcal{R}}\right)$ induced by $\hat{\psi}$. By the definition of direct image sheaf (see, e.g., Chapter F in [23]),

$$
\Gamma\left(W_{0} \times S, \mathcal{B}\right) \cong \Gamma\left(W_{0}, \hat{\mathcal{B}}\right), \quad \Gamma\left(W_{0} \times S, \mathcal{R}\right) \cong \Gamma\left(W_{0}, \hat{\mathcal{R}}\right)
$$

To prove exactness of (7.15) it suffices to show that for every point $x_{0} \in \bar{V}_{0}$, a neighbourhood $W_{0} \subset \bar{V}_{0}$ of $x_{0}$, and a section $\hat{f}_{x_{0}} \in \Gamma\left(W_{0}, \hat{\mathcal{R}}\right)$ there exists a section $\hat{g}_{x_{0}} \in \Gamma\left(\tilde{W}_{0}, \hat{\mathcal{B}}\right)$ over a neighbourhood $\tilde{W}_{0} \subset W_{0}$ of $x_{0}$ such that $\hat{\Psi}_{\tilde{W}_{0}}\left(\hat{g}_{x_{0}}\right)=\left.\hat{f}_{x_{0}}\right|_{\tilde{W}_{0}}$.

Let $f_{x_{0}} \in \Gamma\left(W_{0} \times S, \mathcal{R}\right)$ be the section corresponding to $\hat{f}_{x_{0}}$ under the second isomorphism in (7.16). By definition, a section of sheaf $\mathcal{R}$ over $W_{0} \times S$ is the restriction of a section of $\mathcal{R}$ over an open neighbourhood of $W_{0} \times S$. Therefore, shrinking $W_{0}$, if necessary, we obtain that $f_{x_{0}}$ can be extended to a section of $\mathcal{R}$ over $W_{0} \times M_{1}$, where $M_{1} \subset K$ is an open neighbourhood of $S$. Since $\psi$ is a surjective sheaf homomorphism, for each point $y \in\left\{x_{0}\right\} \times M_{1}$ there exist open sets $W_{0, y} \subset W_{0}, L_{y} \subset M_{1}$ and a section $s_{y} \in \Gamma\left(W_{0, y} \times L_{y}, \mathcal{B}\right)$ such that $y \in$ $W_{0, y} \times L_{y}$ and $\Psi_{W_{0, y} \times L_{y}}\left(s_{y}\right)=\left.f_{x_{0}}\right|_{W_{y} \times L_{y}}$. Since space $\hat{G}_{\mathfrak{a}}\left(\supset M_{1}\right)$ is compact Hausdorff and, hence, is normal, there exists an open subset $M_{2} \subset M_{1}$ such that $S \subset M_{2}$, and $\bar{M}_{2} \subset M_{1}$. Since $\bar{M}_{2}$ is compact, there exist finitely many points $\left\{y_{j}\right\}_{j=1}^{m} \subset S$ such that $\bar{M}_{2} \subset \cup_{j} L_{y_{j}}$. We set $\tilde{L}_{y_{j}}:=\bar{M}_{2} \cap L_{y_{j}}$ for all $j$. There exists a partition of unity $\left\{\rho_{j}\right\} \subset C\left(\bar{M}_{2}\right)$ subordinate to $\left\{\tilde{L}_{y_{j}}\right\}$. We define $\tilde{W}_{0}:=\cap_{j} W_{0, y_{j}}$, and set

$$
g_{x_{0}}(z, \eta):=\sum_{j} \rho_{j}(\eta) s_{y_{j}}(z, \eta), \quad(z, \eta) \in \tilde{W}_{0} \times S .
$$

Then $g_{x_{0}} \in \Gamma\left(\tilde{W}_{0} \times M_{2}, \mathcal{B}\right)$. We have

$$
\Psi_{\tilde{W}_{0} \times S}\left(g_{x_{0}}\right)=\sum_{j} \rho_{j} \Psi_{\tilde{W}_{0} \times \tilde{L}_{y_{j}}}\left(s_{y_{j}}\right)=\left.\sum_{j} \rho_{j} f_{x_{0}}\right|_{\tilde{W}_{0} \times \tilde{L}_{y_{j}}}=\left.f_{x_{0}}\right|_{\tilde{W}_{0} \times S} .
$$

Let $\hat{g}_{x_{0}}$ denote the section in $\Gamma\left(\tilde{W}_{0}, \hat{\mathcal{B}}\right)$ corresponding to $g_{x_{0}}$ under the first isomorphism in (7.16). Then $\hat{\Psi}_{\tilde{W}_{0}}\left(\hat{g}_{x_{0}}\right)=\left.\hat{f}_{x_{0}}\right|_{\tilde{W}_{0}}$, as required. 
Definition 7.12. We say that an analytic sheaf $\mathcal{R}$ (on $U$ ) admits a free resolution of length $N \geq 1$ over $U$ if there exists an exact sequence

$$
\left.\left.\left.\mathcal{F}_{N}\right|_{U} \stackrel{\varphi_{N-1}}{\longrightarrow} \cdots \stackrel{\varphi_{2}}{\longrightarrow} \mathcal{F}_{2}\right|_{U} \stackrel{\varphi_{1}}{\longrightarrow} \mathcal{F}_{1}\right|_{U} \stackrel{\varphi_{0}}{\longrightarrow} \mathcal{R} \longrightarrow 0,
$$

where $\mathcal{F}_{i}$ are free sheaves, i.e., sheaves of the form $\mathcal{O}^{k}$ for some $k \geq 0$ (by definition, $\left.\mathcal{O}^{0}=\{0\}\right)$.

Lemma 7.13. Let $\mathcal{R}$ be an analytic sheaf on $U$ having a free resolution of length $3 N$,

$$
\left.\left.\left.\mathcal{F}_{3 N}\right|_{U} \stackrel{\varphi_{3 N-1}}{\longrightarrow} \cdots \stackrel{\varphi_{2}}{\longrightarrow} \mathcal{F}_{2}\right|_{U} \stackrel{\varphi_{1}}{\longrightarrow} \mathcal{F}_{1}\right|_{U} \stackrel{\varphi_{0}}{\longrightarrow} \mathcal{R} \longrightarrow 0 .
$$

If $N \geq n\left(=\operatorname{dim}_{\mathbb{C}} U_{0}\right)$, then for each $k$ the induced sequence of sections

$$
\Gamma\left(\bar{V}_{k}, \mathcal{F}_{N}\right) \stackrel{\bar{\varphi}_{N-1}}{\longrightarrow} \cdots \stackrel{\bar{\varphi}_{2}}{\longrightarrow} \Gamma\left(\bar{V}_{k}, \mathcal{F}_{2}\right) \stackrel{\bar{\varphi}_{1}}{\longrightarrow} \Gamma\left(\bar{V}_{k}, \mathcal{F}_{1}\right) \stackrel{\bar{\varphi}_{0}}{\longrightarrow} \Gamma\left(\bar{V}_{k}, \mathcal{R}\right) \longrightarrow 0
$$

is exact.

Proof. Let us fix $k \geq 1$. Let $q: \bar{V}_{k} \rightarrow \bar{V}_{0, k}$ be the projection, $q(x, \eta)=x$, $(x, \eta) \in V_{k}:=V_{0, k} \times N_{k}$ (see notation before Definition 7.7). Let $q_{*}$ denote the direct image functor; set $\hat{\mathcal{F}}_{i}:=q_{*}\left(\left.\mathcal{F}_{i}\right|_{\bar{V}_{k}}\right), \hat{\mathcal{R}}:=q_{*}\left(\left.\mathcal{R}\right|_{\bar{V}_{k}}\right), \hat{\varphi}_{i}:=q_{*} \varphi_{i}$. Applying $q_{*}$ to (7.18) we obtain a complex of sheaf homomorphisms

$$
\hat{\mathcal{F}}_{3 N} \stackrel{\hat{\varphi}_{3 N-1}}{\longrightarrow} \cdots \stackrel{\hat{\varphi}_{1}}{\longrightarrow} \hat{\mathcal{F}}_{1} \stackrel{\hat{\varphi}_{0}}{\longrightarrow} \hat{\mathcal{R}} \longrightarrow 0
$$

(a priori this sequence is not exact). By the definition of a direct image sheaf, the sequence of sections of (7.20) over $\bar{V}_{0, k}$ truncated to the $N$-th term,

$$
\Gamma\left(\bar{V}_{0, k}, \hat{\mathcal{F}}_{N}\right) \longrightarrow \cdots \rightarrow \Gamma\left(\bar{V}_{0, k}, \hat{\mathcal{F}}_{1}\right) \longrightarrow \Gamma\left(\bar{V}_{0, k}, \hat{\mathcal{R}}\right) \longrightarrow 0
$$

coincides with sequence (7.19). Hence, the assertion would follow once we proved that sequence $(7.21)$ is exact.

Now, the exact sequence (7.18) yields the collection of short exact sequences

$$
\left.\left.\left.0 \longrightarrow \mathcal{R}_{i}\right|_{\bar{V}_{k}} \stackrel{\iota}{\longrightarrow} \mathcal{F}_{i}\right|_{\bar{V}_{k}} \stackrel{\varphi_{i-1}}{\longrightarrow} \mathcal{R}_{i-1}\right|_{\bar{V}_{k}} \longrightarrow 0, \quad 1 \leq i \leq 3 N-1,
$$

where $\mathcal{R}_{i}:=\operatorname{Im} \varphi_{i}(0 \leq i \leq 3 N-1), \mathcal{R}_{0}:=\mathcal{R}$ and $\iota$ stands for inclusion. We apply to $(7.22)$ the direct image functor $q_{*}$ and Lemma 7.11 to obtain the collection of short exact sequences (recall that $q_{*}$ is left exact, see, e.g., Chapter F in [23])

$$
0 \longrightarrow \mathcal{T}_{i} \stackrel{\hat{\iota}}{\longrightarrow} \hat{\mathcal{F}}_{i} \stackrel{\hat{\varphi}_{i-1}}{\longrightarrow} \mathcal{T}_{i-1} \longrightarrow 0, \quad 1 \leq i \leq 3 N-1,
$$

where $\mathcal{T}_{i}:=q_{*} \mathcal{R}_{i}(0 \leq i \leq 3 N-1)$. An argument similar to that in the proof of Lemma 7.6 implies $H^{l}\left(\bar{V}_{0, k}, \hat{\mathcal{F}}_{i}\right)=0, l \geq 1, k \geq 1,1 \leq i \leq 3 N$. Hence, each short exact sequence (7.23) yields a long exact sequence of the form

$$
\begin{aligned}
0 \longrightarrow \Gamma\left(\bar{V}_{0, k}, \mathcal{T}_{i}\right) \longrightarrow \Gamma\left(\bar{V}_{0, k}, \hat{\mathcal{F}}_{i}\right) \longrightarrow \Gamma\left(\bar{V}_{0, k}, \mathcal{T}_{i-1}\right) \longrightarrow \\
H^{1}\left(\bar{V}_{0, k}, \mathcal{T}_{i}\right) \longrightarrow 0 \longrightarrow H^{1}\left(\bar{V}_{0, k}, \mathcal{T}_{i-1}\right) \longrightarrow H^{2}\left(\bar{V}_{0, k}, \mathcal{T}_{i}\right) \longrightarrow \cdots
\end{aligned}
$$


Thus, $H^{m}\left(\bar{V}_{0, k}, \mathcal{T}_{i}\right) \cong H^{m+1}\left(\bar{V}_{0, k}, \mathcal{T}_{i+1}\right), m \geq 1,1 \leq i \leq 3 N-2$, and so

$$
H^{m}\left(\bar{V}_{0, k}, \mathcal{T}_{i}\right) \cong H^{m+l+1}\left(\bar{V}_{0, k}, \mathcal{T}_{i+l+1}\right), \quad l \geq-1 .
$$

Let us take $m=1,1 \leq i \leq N, l:=2 n-2$. Then

$$
H^{1}\left(\bar{V}_{0, k}, \mathcal{T}_{i}\right) \cong H^{2 n+1}\left(\bar{V}_{0, k}, \mathcal{T}_{i+2 n-1}\right), \quad 1 \leq i \leq N
$$

Since $N \geq n$, we have $i+2 n-1 \leq 3 N-1$ for all $1 \leq i \leq N$; hence, $\mathcal{T}_{i+2 n-1}$ is well defined for all $1 \leq i \leq N$. Since covering dimension of $\bar{V}_{0, k}$ is equal to $2 n$, $H^{2 n+1}\left(\bar{V}_{0, k}, \mathcal{T}_{i+2 n-1}\right)=0$; therefore $H^{1}\left(\bar{V}_{0, k}, \mathcal{T}_{i}\right)=0,1 \leq i \leq N$. Thus, we obtain the collection of short exact sequences

$$
0 \longrightarrow \Gamma\left(\bar{V}_{0, k}, \mathcal{T}_{i}\right) \longrightarrow \Gamma\left(\bar{V}_{0, k}, \hat{\mathcal{F}}_{i}\right) \longrightarrow \Gamma\left(\bar{V}_{0, k}, \mathcal{T}_{i-1}\right) \longrightarrow 0, \quad 1 \leq i \leq N,
$$

which yields exactness of sequence (7.21). The proof is complete.

Lemma 7.14. Let $\mathcal{R}$ be an analytic sheaf on $U$ having a free resolution of length $3 N$

$$
\left.\left.\left.\mathcal{F}_{3 N}\right|_{U} \stackrel{\varphi_{3 N-1}}{\longrightarrow} \cdots \stackrel{\varphi_{2}}{\longrightarrow} \mathcal{F}_{2}\right|_{U} \stackrel{\varphi_{1}}{\longrightarrow} \mathcal{F}_{1}\right|_{U} \stackrel{\varphi_{0}}{\longrightarrow} \mathcal{R} \longrightarrow 0 .
$$

If $N \geq n$, then $\mathcal{R}$ satisfies the Runge condition.

Proof. We have a short exact sequence

$$
\left.0 \longrightarrow \operatorname{Ker} \varphi_{0} \stackrel{\iota}{\longrightarrow} \mathcal{F}_{1}\right|_{U} \stackrel{\varphi_{0}}{\longrightarrow} \mathcal{R} \longrightarrow 0
$$

where $\iota$ stands for inclusion. In the proof of Lemma 7.13 we have shown that, under the present assumptions, for each $k \geq 1$ the sequence of sections

$$
0 \longrightarrow \Gamma\left(\bar{V}_{k}, \text { Ker } \varphi_{0}\right) \stackrel{\bar{\iota}}{\longrightarrow} \Gamma\left(\bar{V}_{k}, \mathcal{F}_{1}\right) \stackrel{\bar{\varphi}_{0}}{\longrightarrow} \Gamma\left(\bar{V}_{k}, \mathcal{R}\right) \longrightarrow 0
$$

is exact. Given a section $h \in \Gamma\left(\bar{V}_{k}, \mathcal{F}_{1}\right), \mathcal{F}_{1}:=\mathcal{O}^{m_{1}}$ for some $m_{1} \in \mathbb{Z}_{+}$, we define semi-norm $|h|_{k}:=\sup _{x \in \bar{V}_{k}}\|\hat{h}(x)\|$, where $\|\cdot\|$ is the Euclidean norm in $\mathbb{C}^{m_{1}}$, and $\hat{h}(\omega) \in \mathbb{C}^{m}$ denotes the value of germ $h(\omega)$ (i.e. the common value of its representatives) at $\omega \in U$. Now, for a section $h \in \Gamma\left(\bar{V}_{k}, \mathcal{R}\right)$ we set

$$
|f|_{k}:=\inf _{h}\left\{|h|_{k}: h \in \Gamma\left(\bar{V}_{k}, \mathcal{F}_{1}\right), \bar{\varphi}_{0}(h)=f\right\} .
$$

We obtain a family of semi-norms $\left\{|\cdot|_{k}: k \geq 1\right\}$ on $\Gamma(U, \mathcal{R})$. Let us show that for this family of semi-norms conditions (a)-(d) of Definition 7.7 are satisfied.

(a) Let $f \in \Gamma\left(\bar{V}_{k}, \mathcal{R}\right)$. There exists a section $h \in \Gamma\left(\bar{V}_{k}, \mathcal{F}_{1}\right)$ such that $f=\bar{\varphi}_{0}(h)$. Using the same argument as in the proof of Lemma 7.9 , we obtain that for any $\varepsilon>0$ there exists a section $\tilde{h} \in \Gamma\left(U, \mathcal{F}_{1}\right)$ such that $|\tilde{h}-h|_{k}<\varepsilon$. We set $\tilde{f}:=$ $\bar{\varphi}_{0}(\tilde{h}) \in \Gamma(U, \mathcal{R})$. By definition, $|\tilde{f}-f|_{k}<\varepsilon$, as required.

(b) Let $f \in \Gamma\left(\bar{V}_{k+1}, \mathcal{R}\right)$. Since

$$
\left.\left\{h \in \Gamma\left(\bar{V}_{k+1}, \mathcal{F}_{1}\right), f=\bar{\varphi}_{0}(h)\right\}\right|_{\bar{V}_{k}} \subset\left\{g \in \Gamma\left(\bar{V}_{k}, \mathcal{F}_{1}\right),\left.f\right|_{\bar{V}_{k}}=\bar{\varphi}_{0}(g)\right\}
$$

and $|h|_{k} \leq|h|_{k+1}$ for every $h \in \Gamma\left(\bar{V}_{k+1}, \mathcal{F}_{1}\right)$, condition (b) is satisfied with $M_{k}=1$ $(k \geq 1)(\operatorname{see}(7.25))$. 
(c) Let $\left\{f_{j}\right\}$ be a Cauchy sequence in $\Gamma\left(\bar{V}_{k+1}, \mathcal{R}\right)$. We must show that $\left\{\left.f_{j}\right|_{\bar{V}_{k}}\right\}$ has a limit in the space $\left(\Gamma\left(\bar{V}_{k}, \mathcal{R}\right),|\cdot|_{k}\right)$. In fact, there exists a Cauchy sequence $\left\{h_{j}\right\} \subset \Gamma\left(\bar{V}_{k+1}, \mathcal{O}^{m_{1}}\right)$ such that $f_{j}=\bar{\varphi}_{0}\left(h_{j}\right)$ for all $j$. Clearly, there exists a function $h \in \mathcal{O}\left(V_{k+1}, \mathbb{C}^{m_{1}}\right) \cap C\left(\bar{V}_{k+1}, \mathbb{C}^{m_{1}}\right)$ such that

$$
\sup _{\omega \in \bar{V}_{k+1}}\left|h(\omega)-\hat{h}_{j}(\omega)\right| \rightarrow 0 \quad \text { as } j \rightarrow \infty .
$$

Then $h \in \Gamma\left(\bar{V}_{k}, O^{m_{1}}\right)$ and by (7.26) $\left|h-h_{j}\right|_{k} \rightarrow 0$ as $j \rightarrow \infty$. Now, we set $f:=\bar{\varphi}_{0}(h) \in \Gamma\left(\bar{V}_{k}, \mathcal{R}\right)$, so by continuity $\left|f-f_{j}\right|_{k} \rightarrow 0$ as $j \rightarrow \infty$.

(d) Let $f \in \Gamma\left(\bar{V}_{k+1}, \mathcal{R}\right),|f|_{k+1}=0$. We must show that $\left.f\right|_{\bar{V}_{k}}=0$. Indeed, by definition, there exists a sequence of sections $h_{l} \in \Gamma\left(\bar{V}_{k+1}, \mathcal{F}_{1}\right)$ such that $f=\bar{\varphi}_{0}\left(h_{l}\right)$ for all $l$ and $\sup _{\omega \in \bar{V}_{k+1}}\left\|\hat{h}_{l}(\omega)\right\| \rightarrow 0$ as $l \rightarrow \infty$. Let $g_{l}:=h_{1}-h_{l}, l \geq 1$. Then $g_{l} \in \Gamma\left(\bar{V}_{k+1}, \operatorname{Ker} \varphi_{0}\right)$ and

$$
\hat{g}_{l}(\omega) \rightarrow \hat{h}_{1}(\omega) \text { uniformly in } \omega \in \bar{V}_{k+1} \text { as } l \rightarrow \infty .
$$

Now, suppose to the contrary that $\left.f\right|_{\bar{V}_{k}} \neq 0$. Then $\left.h_{1}\right|_{\bar{V}_{k}} \notin \Gamma\left(\bar{V}_{k}, \operatorname{Ker} \varphi_{0}\right)$.

Consider the second fragment of the free resolution of $\mathcal{R}$ :

$$
\left.0 \longrightarrow \operatorname{Ker} \varphi_{1} \stackrel{\iota}{\longrightarrow} \mathcal{F}_{2}\right|_{U} \stackrel{\varphi_{1}}{\longrightarrow} \operatorname{Ker} \varphi_{0} \longrightarrow 0,
$$

and the corresponding sequence of sections (see Lemma 7.13)

$$
0 \longrightarrow \Gamma\left(\bar{V}_{k+1}, \operatorname{Ker} \varphi_{1}\right) \stackrel{\bar{\iota}}{\longrightarrow} \Gamma\left(\bar{V}_{k+1}, \mathcal{F}_{2}\right) \stackrel{\bar{\varphi}_{1}}{\longrightarrow} \Gamma\left(\bar{V}_{k+1}, \operatorname{Ker} \varphi_{0}\right) \longrightarrow 0
$$

where $\bar{\varphi}_{1}$ is given by a matrix with entries in $\Gamma\left(\bar{V}_{k+1}, \mathcal{O}\right)$.

Recall that $\Gamma\left(\bar{V}_{k+1}, \mathcal{F}_{2}\right)$ is endowed with sup-norm

$$
|g|_{k+1}=\sup _{\omega \in \bar{V}_{k+1}}\|\hat{g}(\omega)\|, \quad g \in \Gamma\left(\bar{V}_{k+1}, \mathcal{F}_{2}\right) .
$$

Each section in the space $\Gamma\left(\bar{V}_{k+1}, \mathcal{F}_{2}\right)$ determines a continuous function on $\bar{V}_{k+1}$ holomorphic in $V_{k+1}$. Let $\mathcal{A}\left(\bar{V}_{k+1}, \mathcal{F}_{2}\right)$ denote the completion of the space of these functions with respect to norm (7.30). Analogously, we endow the space $\Gamma\left(\bar{V}_{k+1}, \mathcal{F}_{1}\right)$ with sup-norm and denote by $\mathcal{A}\left(\bar{V}_{k+1}, \operatorname{Ker} \varphi_{0}\right)$ the completion (with respect to this norm) of its subspace $\Gamma\left(\bar{V}_{k+1}, \operatorname{Ker} \varphi_{0}\right)$. Then (7.29) yields an exact sequence of Banach spaces

$$
\mathcal{A}\left(\bar{V}_{k+1}, \mathcal{F}_{2}\right) \stackrel{\bar{\varphi}_{1}}{\longrightarrow} \mathcal{A}\left(\bar{V}_{k+1}, \operatorname{Ker} \varphi_{0}\right) \longrightarrow 0 .
$$

It follows from (7.27) that $\left\{g_{l}\right\}$, with $g_{l}$ viewed as functions in $\mathcal{A}\left(\bar{V}_{k+1}, \operatorname{Ker} \varphi_{0}\right)$, is a Cauchy sequence and hence has a limit $g \in \mathcal{A}\left(\bar{V}_{k+1}, \operatorname{Ker} \varphi_{0}\right)$. Then there exists $r \in \mathcal{A}\left(\bar{V}_{k+1}, \mathcal{F}_{2}\right)$ such that $g=\bar{\varphi}_{1}(r)$. Also, by Lemma 7.13 we obtain that sequence (7.28) induces an exact sequence of sections

$$
0 \longrightarrow \Gamma\left(\bar{V}_{k}, \operatorname{Ker} \varphi_{1}\right) \stackrel{\bar{\iota}_{\bar{V}_{k}}}{\longrightarrow} \Gamma\left(\bar{V}_{k}, \mathcal{F}_{2}\right) \stackrel{\left.\bar{\varphi}_{1}\right|_{\bar{V}_{k}}}{\longrightarrow} \Gamma\left(\bar{V}_{k}, \operatorname{Ker} \varphi_{0}\right) \longrightarrow 0 .
$$

Clearly, we have

$$
\left.A\left(\bar{V}_{k+1}, \mathcal{F}_{2}\right)\right|_{\bar{V}_{k}} \subset \Gamma\left(\bar{V}_{k}, \mathcal{F}_{2}\right) \text { and }\left.\mathcal{A}\left(\bar{V}_{k+1}, \operatorname{Ker} \varphi_{0}\right)\right|_{\bar{V}_{k}} \subset \Gamma\left(\bar{V}_{k}, \operatorname{Ker} \varphi_{0}\right) .
$$


Hence, $\left.r\right|_{\bar{V}_{k}} \in \Gamma\left(\bar{V}_{k}, \mathcal{F}_{2}\right)$ and $\left.g\right|_{\bar{V}_{k}}=\bar{\varphi}_{1}\left(\left.r\right|_{\bar{V}_{k}}\right) \in \Gamma\left(\bar{V}_{k}\right.$, Ker $\left.\varphi_{0}\right)$. Since, by our definition, $\left.h_{1}\right|_{\bar{V}_{k}}=\left.g\right|_{\bar{V}_{k}}$, we have $\left.h_{1}\right|_{\bar{V}_{k}} \in \Gamma\left(V_{k}, \operatorname{Ker} \varphi_{0}\right)$, which contradicts the assumption $\left.f\right|_{\bar{V}_{k}} \neq 0$.

Lemma 7.15. Let $\mathcal{R}$ be an analytic sheaf over $U$ admitting a free resolution of length $4 N$

$$
\left.\left.\left.\mathcal{F}_{4 N}\right|_{U} \stackrel{\varphi_{4 N-1}}{\longrightarrow} \cdots \stackrel{\varphi_{2}}{\longrightarrow} \mathcal{F}_{2}\right|_{U} \stackrel{\varphi_{1}}{\longrightarrow} \mathcal{F}_{1}\right|_{U} \stackrel{\varphi_{0}}{\longrightarrow} \mathcal{R} \longrightarrow 0 .
$$

If $N \geq n$, and for each $k$, the sequence of sections

$$
\Gamma\left(\bar{V}_{k}, \mathcal{F}_{N}\right) \stackrel{\bar{\varphi}_{N-1}}{\longrightarrow} \cdots \stackrel{\bar{\varphi}_{2}}{\longrightarrow} \Gamma\left(\bar{V}_{k}, \mathcal{F}_{2}\right) \stackrel{\bar{\varphi}_{1}}{\longrightarrow} \Gamma\left(\bar{V}_{k}, \mathcal{F}_{1}\right) \stackrel{\bar{\varphi}_{0}}{\longrightarrow} \Gamma\left(\bar{V}_{k}, \mathcal{R}\right) \longrightarrow 0
$$

is exact, then the sequence of sections

$$
\Gamma\left(U, \mathcal{F}_{N}\right) \stackrel{\bar{\varphi}_{N-1}}{\longrightarrow} \cdots \stackrel{\bar{\varphi}_{2}}{\longrightarrow} \Gamma\left(U, \mathcal{F}_{2}\right) \stackrel{\bar{\varphi}_{1}}{\longrightarrow} \Gamma\left(U, \mathcal{F}_{1}\right) \stackrel{\bar{\varphi}_{0}}{\longrightarrow} \Gamma(U, \mathcal{R}) \longrightarrow 0
$$

is also exact.

Proof. Exact sequence (7.31) yields the collection of short exact sequences

$$
\left.0 \longrightarrow \mathcal{R}_{i} \stackrel{\iota}{\longrightarrow} \mathcal{F}_{i}\right|_{U} \stackrel{\varphi_{i-1}}{\longrightarrow} \mathcal{R}_{i-1} \longrightarrow 0, \quad 1 \leq i \leq N-1
$$

where $\mathcal{R}_{i}:=\operatorname{Im} \varphi_{i}(0 \leq i \leq N-1), \mathcal{R}_{0}:=\mathcal{R}$, and $\iota$ stands for inclusion. Recall that the section functor $\Gamma$ is left exact (see, e.g., Chapter 3 in [23]), hence we have the collection of exact sequences

$$
0 \longrightarrow \Gamma\left(U, \mathcal{R}_{i}\right) \stackrel{\bar{\iota}}{\longrightarrow} \Gamma\left(U, \mathcal{F}_{i}\right) \stackrel{\bar{\varphi}_{i-1}}{\longrightarrow} \Gamma\left(U, \mathcal{R}_{i-1}\right), \quad 1 \leq i \leq N-1
$$

It suffices to show that $\bar{\varphi}_{i-1}$ is surjective; this would imply that (7.33) is exact.

It follows from the exactness of sequence (7.32) that for each $k$, the sequences

$$
0 \longrightarrow \Gamma\left(\bar{V}_{k}, \mathcal{R}_{i}\right) \stackrel{\bar{\iota}}{\longrightarrow} \Gamma\left(\bar{V}_{k}, \mathcal{F}_{i}\right) \stackrel{\bar{\varphi}_{i-1}}{\longrightarrow} \Gamma\left(\bar{V}_{k}, \mathcal{R}_{i-1}\right) \longrightarrow 0, \quad 1 \leq i \leq N-1,
$$

are exact. By Lemma $7.6 H^{1}\left(\bar{V}_{k}, \mathcal{F}_{i}\right)=0,1 \leq i \leq N$, for all $k \geq 1$, therefore the long exact sequence induced by (7.34) over $\bar{V}_{k}$ has the form

$$
\begin{aligned}
& 0 \longrightarrow \Gamma\left(\bar{V}_{k}, \mathcal{R}_{i}\right) \longrightarrow \Gamma\left(\bar{V}_{k}, \mathcal{F}_{i}\right) \rightarrow \Gamma\left(\bar{V}_{k}, \mathcal{R}_{i-1}\right) \longrightarrow \\
& H^{1}\left(\bar{V}_{k}, \mathcal{R}_{i}\right) \longrightarrow 0 \longrightarrow H^{1}\left(\bar{V}_{k}, \mathcal{R}_{i-1}\right) \longrightarrow H^{2}\left(\bar{V}_{k}, \mathcal{R}_{i}\right) \longrightarrow \cdots, \quad 1 \leq i \leq N-1
\end{aligned}
$$

Now it follows from $(7.35)$ that $H^{1}\left(\bar{V}_{k}, \mathcal{R}_{i}\right)=0$ for all $k \geq 1,1 \leq i \leq N-1$.

The long exact sequence induced by (7.34) over $U$ has the form

$$
\begin{aligned}
& (7.36) \quad 0 \longrightarrow \Gamma\left(U, \mathcal{R}_{i}\right) \longrightarrow \Gamma\left(U, \mathcal{F}_{i}\right) \rightarrow \Gamma\left(U, \mathcal{R}_{i-1}\right) \longrightarrow \\
& H^{1}\left(U, \mathcal{R}_{i}\right) \longrightarrow H^{1}\left(U, \mathcal{F}_{i}\right) \longrightarrow H^{1}\left(U, \mathcal{R}_{i-1}\right) \longrightarrow H^{2}\left(U, \mathcal{R}_{i}\right) \rightarrow \cdots, \quad 1 \leq i \leq N-1
\end{aligned}
$$


Each sheaf $\mathcal{R}_{i}, 1 \leq i \leq N-1$, has a free resolution of length $3 N$, hence by Lemma 7.14 it satisfies the Runge condition. It follows from Lemma 7.8 (2) that $H^{1}\left(U, \mathcal{R}_{i}\right)=0$ for all $1 \leq i \leq N-1$. We obtain from (7.36) that sequences

$$
0 \longrightarrow \Gamma\left(U, \mathcal{R}_{i}\right) \stackrel{\bar{\iota}}{\longrightarrow} \Gamma\left(U, \mathcal{F}_{i}\right) \stackrel{\bar{\varphi}_{i-1}}{\longrightarrow} \Gamma\left(U, \mathcal{R}_{i-1}\right) \longrightarrow 0, \quad 1 \leq i \leq N-1,
$$

are exact, which implies exactness of sequence (7.33).

Proof of Proposition 7.1. (1) Follows from Lemmas 7.13 and 7.15.

(2) According to Lemma 7.14 sheaf $\mathcal{R}$ satisfies the Runge condition. Hence, by Lemma 7.8 we only have to show that $H^{i}\left(\bar{V}_{k}, \mathcal{R}\right)=0$ for all $i \geq 1$ and $k \geq 1$.

Let $\mathcal{V}$ be an open cover of $\bar{V}_{k}:=\bar{V}_{0, k} \times \bar{K}$. It suffices to show that given an $i$-cocycle $\sigma \in \mathcal{Z}^{i}(\mathcal{V}, \mathcal{R})$ (see notation before Lemma 7.6) there exists a refinement $\mathcal{V}^{\prime}$ of $\mathcal{V}$ such that the image of $\sigma$ by the refinement map $\mathcal{Z}^{i}(\mathcal{V}, \mathcal{R}) \rightarrow \mathcal{Z}^{i}\left(\mathcal{V}^{\prime}, \mathcal{R}\right)$ belongs to $\mathcal{B}^{i}\left(\mathcal{V}^{\prime}, \mathcal{R}\right)$.

By Lemma $7.5(1)$ there exists a finite refinement $\mathcal{U}=\left\{U_{\alpha}\right\}, U_{\alpha}:=U_{0, l} \times L_{j}$, $\alpha=(l, j)$, of cover $\mathcal{V}$ of class $(P)$ (see Definition 7.4). Let $s=s_{\mathcal{U}}$ be the number of elements of $\mathcal{U}$ and let $N \geq \max \{n, s\}$ be the length of the free resolution of $\mathcal{R}$ over $U$. By the definition of an open cover of class $(P)$, a section of sheaf $\mathcal{R}$ over an element $U_{\alpha}$ of $\mathcal{U}$ admits an extension to $\tilde{U}_{\alpha}=\tilde{U}_{0, l} \times L_{j}$, where $\tilde{U}_{0, l}=$ $\tilde{U}_{0}^{1} \times \cdots \times \tilde{U}_{0}^{n} \Subset U_{0}$ is a product domain such that each $\tilde{U}_{0}^{i} \subset \mathbb{C}(1 \leq i \leq n)$ is simply connected and has smooth boundary, and $U_{0, l}=\bar{V}_{0, k} \cap \tilde{U}_{0, l}$. By part (1) of the proposition, over each $U_{\alpha}$ the sequence of sections $U_{\alpha}$ corresponding to (7.1) is exact (there we can take product domain $\tilde{U}_{0, l}$ instead of polydisk $U_{0}$ ). Hence, we have a sequence of cochain complexes

$$
\mathcal{C} \cdot\left(\mathcal{U}, \mathcal{F}_{N}\right) \rightarrow \cdots \rightarrow \mathcal{C} \cdot\left(\mathcal{U}, \mathcal{F}_{1}\right) \rightarrow \mathcal{C} \cdot(\mathcal{U}, \mathcal{R}) \rightarrow 0
$$

By Lemma $7.5(2)$ there exists a refinement $\mathcal{U}^{\prime}$ of cover $\mathcal{U}$ of class $(P)$ of the same cardinality. We have a commutative diagram with exact rows

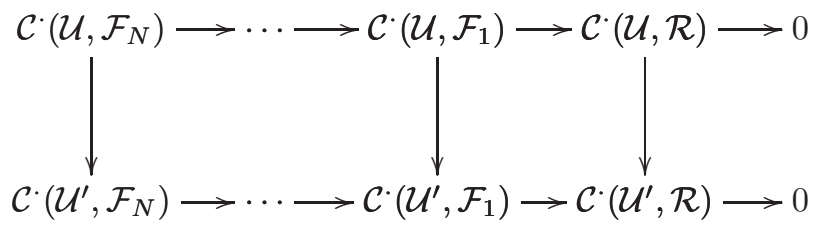

or, equivalently, the collection of commutative diagrams with exact rows

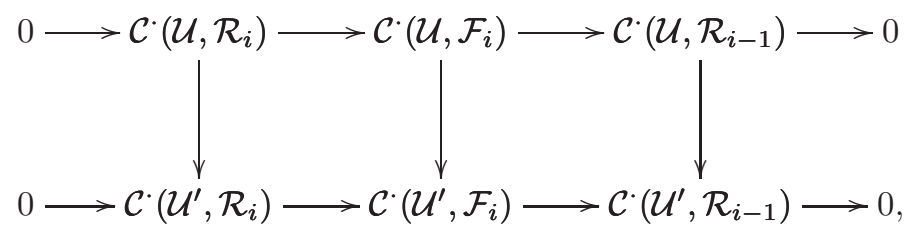

where $\mathcal{R}_{i}:=\operatorname{Im} \varphi_{i}(0 \leq i \leq N-1), \mathcal{R}_{0}:=\mathcal{R}$. 
In turn, each row yields the long exact sequence

$$
\begin{aligned}
& 0 \longrightarrow \Gamma\left(\bar{V}_{k}, \mathcal{R}_{i}\right) \longrightarrow \Gamma\left(\bar{V}_{k}, \mathcal{F}_{i}\right) \rightarrow \Gamma\left(\bar{V}_{k}, \mathcal{R}_{i-1}\right) \longrightarrow \\
& H^{1}\left(\mathcal{U}, \mathcal{R}_{i}\right) \longrightarrow H^{1}\left(\mathcal{U}, \mathcal{F}_{i}\right) \stackrel{\varphi_{i-1}^{1}}{\longrightarrow} H^{1}\left(\mathcal{U}, \mathcal{R}_{i-1}\right) \stackrel{\psi_{i}^{2}}{\longrightarrow} H^{2}\left(\mathcal{U}, \mathcal{R}_{i}\right) \longrightarrow \cdots, 1 \leq i \leq N-1,
\end{aligned}
$$

(and a similar one for $\mathcal{U}^{\prime}$ ), where $H^{l}\left(\mathcal{U}, \mathcal{R}_{i}\right):=\mathcal{Z}^{l}\left(\mathcal{U}, \mathcal{R}_{i}\right) / \mathcal{B}^{l}\left(\mathcal{U}, \mathcal{R}_{i}\right)$ are the Cech cohomology groups corresponding to cover $\mathcal{U}$. These sequences form the commutative diagram

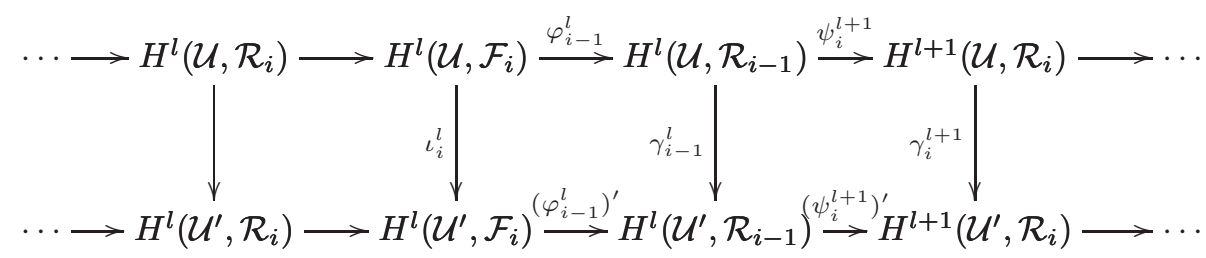

where $\iota_{i}^{l}, \gamma_{i-1}^{l}, \gamma_{i}^{l+1}$ are the corresponding refinement maps.

We have to show that given $\sigma \in H^{l}(\mathcal{U}, \mathcal{R}), l \geq 1$, there exists a refinement $\mathcal{W}$ of cover $\mathcal{U}$ such that the image of $\sigma$ in $H^{l}(\mathcal{W}, \mathcal{R})$ is zero. We construct this refinement using the following algorithm.

Suppose that there exists a non-zero $\sigma \in H^{l}\left(\mathcal{U}, \mathcal{R}_{i-1}\right)$. Consider the following case:

$\left.{ }^{*}\right) \psi_{i}^{l+1}(\sigma)=0$. Then there exists $\eta \in H^{l}\left(\mathcal{U}, \mathcal{F}_{i}\right)$ such that $\sigma=\varphi_{i-1}^{l}(\eta)$. We have $\gamma_{i-1}^{l}(\sigma)=\left(\varphi_{i-1}^{l}\right)^{\prime}\left(\iota_{i}^{l}(\eta)\right)$. By Lemma $7.6 \iota_{i}^{l}\left(H^{l}\left(\mathcal{U}, \mathcal{F}_{i}\right)\right)=0$, hence the image of $\sigma$ by the refinement map $\gamma_{i-1}^{l}(\sigma)=0 \in H^{l}\left(\mathcal{U}^{\prime}, \mathcal{R}_{i-1}\right)$.

We start with $\mathcal{R}_{0}=\mathcal{R}$ assuming that there exists a non-zero $\sigma \in H^{l}(\mathcal{U}, \mathcal{R})$, $l \geq 1$. If case $\left(^{*}\right)$ occurs we set $\mathcal{W}:=\mathcal{U}^{\prime}$. For otherwise, there exists $2 \leq k \leq s$ such that $\left(\psi_{k}^{l+k} \circ \cdots \circ \psi_{1}^{l+1}\right)(\sigma)=0 \in H^{l+k}\left(\mathcal{U}, \mathcal{R}_{k}\right)$. (Indeed, assuming the opposite we obtain a non-zero element of $H^{l+s}\left(\mathcal{U}, \mathcal{R}_{s}\right)$; however, since the cardinality of $\mathcal{U}$ is $s$, we have $H^{l+s}\left(\mathcal{U}, \mathcal{R}_{s}\right)=0$, a contradiction.) Thus case $\left(^{*}\right)$ occurs for $\tilde{\sigma}:=$ $\left(\psi_{k-1}^{l+k-1} \circ \cdots \circ \psi_{1}^{l+1}\right)(\sigma)$ instead of $\sigma$ which implies that the image of $\tilde{\sigma}$ under the refinement map $H^{l+k-1}\left(\mathcal{U}, \mathcal{R}_{k-1}\right) \rightarrow H^{l+k-1}\left(\mathcal{U}^{\prime}, \mathcal{R}_{k-1}\right)$ is zero. Further, starting with cover $\mathcal{U}^{\prime}$ (instead of $\mathcal{U}$ ) and applying consequently case $\left(^{*}\right)$ to images of $\left(\psi_{p}^{l+p} \circ \cdots \circ \psi_{1}^{l+1}\right)(\sigma), p=k-2, \ldots, 1$, under the corresponding refinement maps we finally obtain the required refinement $\mathcal{W}$ of $\mathcal{U}$ such that the image of $\sigma$ under the refinement map $H^{l}\left(\mathcal{U}, \mathcal{R}_{0}\right) \rightarrow H^{l}\left(\mathcal{W}, \mathcal{R}_{0}\right)$ is zero.

\subsection{Proof of Proposition 7.2}

The proof is based on the following lemma.

Lemma 7.16. Let $U_{0} \Subset \mathbb{C}^{n}$ be an open polydisk, and $K_{1}, K_{2} \in \mathfrak{Q}$. Let $\mathcal{R}$ be an analytic sheaf over $U_{0} \times\left(K_{1} \cup K_{2}\right)$. Let $x_{0} \in U_{0}$.

Suppose that for every $N \geq 1$ sheaf $\mathcal{R}$ admits free resolutions of length $N$ over $U_{0} \times K_{1}$ and $U_{0} \times K_{2}$. Then for any open subsets $L_{1} \Subset K_{1}, L_{2} \Subset K_{2}$ such that $L_{i} \in \mathfrak{Q}(i=1,2)$, there exists an open neighbourhood $V_{0} \subset U_{0}$ of $x_{0}$ such that for every $N \geq 1$ sheaf $\mathcal{R}$ admits a free resolution of length $N$ over $V_{0} \times\left(L_{1} \cup L_{2}\right)$. 
We prove Lemma 7.16 in the next subsection but now we use it in the proof of the proposition.

Proof of Proposition 7.2. Let $U_{0} \Subset \mathbb{C}^{n}$ be an open polydisk, $x_{0} \in U_{0}$. Since sets $\bar{p}^{-1}\left(U_{0}\right)$ and $U_{0} \times \hat{G}_{\mathfrak{a}}$ are biholomorphic (see subsection 4.2 ), it suffices to prove the proposition for a coherent sheaf $\mathcal{A}$ over $U_{0} \times \hat{G}_{\mathfrak{a}}$.

By the definition of a coherent sheaf (see (2.4)), there exists a finite open cover of $\left\{x_{0}\right\} \times \hat{G}_{\mathfrak{a}}$ by sets $W_{0, i} \times L_{i}$, where $W_{0, i} \subset U_{0}$ is an open neighbourhood of $x_{0}$, $\cup_{i} L_{i}=\hat{G}_{\mathfrak{a}}$, and for every $N \geq 1$ sheaf $\mathcal{A}$ admits free resolutions of length $N$ over each $W_{0, i} \times L_{i}$.

By Lemma 5.2 there exists a collection of finite refinements

$$
\mathcal{L}^{k}(m)=\left\{L_{j}^{k}: L_{j}^{k} \in \mathfrak{Q}, 1 \leq j \leq m\right\}, \quad k \geq 1,
$$

of open cover $\left\{L_{i}\right\}$ such that $L_{j}^{k+1} \Subset L_{j}^{k}$ for all $1 \leq j \leq m, k \geq 1$.

Let $k=1$. We apply Lemma 7.16 to sheaf $\mathcal{A}$ with $K_{1}:=L_{m-1}^{1}, K_{2}:=L_{m}^{1}$, $L_{1}:=L_{m-1}^{1}, L_{2}:=L_{m-1}^{2}$ to obtain an open neighbourhood $V_{0}:=V_{0, m} \subset \cap_{i} W_{0, i}$ of $x_{0}$ such that for each $N \geq 1$ sheaf $\mathcal{A}$ has a free resolution of length $N$ over $V_{0, m} \times\left(L_{m-1}^{2} \cup L_{m-1}^{2}\right)$.

Next, we set

$$
\mathcal{L}^{k}(m-1):=\left\{L_{1}^{k}, \ldots, L_{m-2}^{k}, \tilde{L}_{m-1}^{k}\right\}, \quad \tilde{L}_{m-1}^{k}:=L_{m-1}^{k} \cup L_{m}^{k}, \quad k \geq 2 .
$$

Taking $k=2$ we apply an argument similar to the above to the cover $\mathcal{L}^{2}(m-1)$ of $\hat{G}_{\mathfrak{a}}$ obtaining that for each $N \geq 1$ sheaf $\mathcal{A}$ has a free resolution of length $N$ over $V_{0, m-1} \times\left(L_{m-2}^{3} \cup \tilde{L}_{m-1}^{3}\right)$ for some open neighbourhood $V_{0, m-1} \subset V_{0, m}$ of $x_{0}$. Then we define

$$
\mathcal{L}^{k}(m-2):=\left\{L_{1}^{k}, \ldots, L_{m-3}^{k}, \tilde{L}_{m-2}^{k}\right\}, \quad \tilde{L}_{m-2}^{k}:=L_{m-2}^{k} \cup \tilde{L}_{m-1}^{k}, \quad k \geq 3, \text { etc. }
$$

After $m-1$ steps we obtain that there exists an open neighbourhood $V_{0,1} \subset$ $\bigcap_{i} W_{0, i}$ of $x_{0}$ such that for each $N \geq 1$ sheaf $\mathcal{A}$ has a free resolution over $V_{0,1} \times \hat{G}_{\mathfrak{a}}$, as required.

\subsection{Proof of Lemma 7.16}

We will use the following notation.

Let $M_{l \times k}(\mathbb{C})$ be the space of $l \times k$ matrices $C=\left(c_{i j}\right)$ with entries $c_{i j} \in \mathbb{C}$ endowed with norm $|C|:=\max \left\{\left|c_{i j}\right|\right\}_{i, j=1}^{l, k}$. We set $M_{k}(\mathbb{C}):=M_{k \times k}(\mathbb{C})$.

Let $G L_{k}(\mathbb{C}) \subset M_{k}(\mathbb{C})$ be the group of invertible matrices. We denote by $I=I_{k} \in G L_{k}(\mathbb{C})$ the identity matrix.

Let $U_{0} \subset \mathbb{C}^{n}$ be an open polydisk, $K \in \mathfrak{Q}$; set $U:=U_{0} \times K$. The space $\mathcal{O}\left(U, M_{k}(\mathbb{C})\right)$ of holomorphic $M_{k}(\mathbb{C})$-valued functions is endowed with norm

$$
\|F\|_{U}:=\sup _{x \in U}|F(x)|, \quad F \in \mathcal{O}\left(U, M_{k}(\mathbb{C})\right) .
$$

The subset $\mathcal{O}\left(U, G L_{k}(\mathbb{C})\right) \subset \mathcal{O}\left(U, M_{k}(\mathbb{C})\right)$ of holomorphic $G L_{k}(\mathbb{C})$-valued maps on $U$ has the induced topology of uniform convergence on compact subsets of $U$ (see Lemma $5.3(2)$ ). 
The identity map $(z, \omega) \rightarrow I,(z, \omega) \in U$, will be denoted also by $I$.

Lemma 7.17. Let $U^{\prime}:=U_{0} \times K^{\prime}, U^{\prime \prime}:=U_{0} \times K^{\prime \prime}$, where $K^{\prime}, K^{\prime \prime} \in \mathfrak{Q}$. Suppose that $H \in \mathcal{O}\left(U^{\prime} \cap U^{\prime \prime}, G L_{k}(\mathbb{C})\right)$ belongs to the connected component of the identity map $I$ in $\mathcal{O}\left(U^{\prime} \cap U^{\prime \prime}, G L_{k}(\mathbb{C})\right)$.

Then for any open polydisk $V_{0} \Subset U_{0}$ and open subsets $L^{\prime} \Subset K^{\prime}, L^{\prime \prime} \Subset K^{\prime \prime}$ there exists a function $H^{\prime} \in \mathcal{O}\left(V^{\prime} \cup V^{\prime \prime}, G L_{k}(\mathbb{C})\right)$, where $V^{\prime}:=V_{0} \times L^{\prime}, V^{\prime \prime}:=V_{0} \times L^{\prime \prime}$, such that $\left.H^{\prime}\right|_{V^{\prime} \cap V^{\prime \prime}}=\left.H\right|_{V^{\prime} \cap V^{\prime \prime}}$.

Proof. We may assume without loss of generality that polydisks $V_{0}, U_{0}$ are centered at the origin $0 \in \mathbb{C}^{n}$.

First, suppose that $\|I-H\|_{V^{\prime} \cap V^{\prime \prime}}<1 / 2$. Then we can define $F:=\log H=$ $-\sum_{j=1}^{\infty} \frac{(I-H)^{j}}{j} \in \mathcal{O}\left(V^{\prime} \cap V^{\prime \prime}, M_{k}(\mathbb{C})\right) \cap C\left(\bar{V}^{\prime} \cap \bar{V}^{\prime \prime}, M_{k}(\mathbb{C})\right)$. Let us show that there exists a function $F^{\prime} \in \mathcal{O}\left(V^{\prime} \cup V^{\prime \prime}, M_{k}(\mathbb{C})\right.$ such that $\left.F^{\prime}\right|_{V^{\prime} \cap V^{\prime \prime}}=\left.F\right|_{V^{\prime} \cap V^{\prime \prime}}$. Indeed, we can expand the $C\left(\bar{L}^{\prime} \cap \bar{L}^{\prime \prime}, M_{k}(\mathbb{C})\right)$-valued holomorphic function $F(z, \cdot)$ in the Taylor series about 0 ,

$$
F(z, \eta)=\sum_{m=0}^{\infty} b_{m}(\eta) z^{m}, \quad z \in \tilde{V}_{0}, \quad \eta \in \bar{L}^{\prime} \cap \bar{L}^{\prime \prime},
$$

where $b_{m} \in C\left(\bar{L}^{\prime} \cap \bar{L}^{\prime \prime}, M_{k}(\mathbb{C})\right)$ and $\tilde{V}_{0}$ is an open neighbourhood of $\bar{V}_{0}$. Note that $\bar{L}^{\prime} \cup \bar{L}^{\prime \prime}$ is compact (as a closed subspace of compact space $\hat{G}_{\mathfrak{a}}$ ), and hence is normal. Therefore, using the Tietze-Urysohn extension theorem, we can extend each $b_{m}$ to a function $\tilde{b}_{m} \in C\left(\bar{L}^{\prime} \cup \bar{L}^{\prime \prime}, M_{k}(\mathbb{C})\right)$ such that $\sup _{\omega \in \bar{L}^{\prime} \cap \bar{L}^{\prime \prime}}\left|b_{m}(\omega)\right|=$ $\sup _{\omega \in \bar{L}^{\prime} \cup \bar{L}^{\prime \prime}}\left|\tilde{b}_{m}(\omega)\right|$. Then we define

$$
F^{\prime}(z, \omega):=\sum_{m=0}^{\infty} \tilde{b}_{m}(\omega) z^{m}, \quad z \in V_{0}, \quad \omega \in L^{\prime} \cup L^{\prime \prime} .
$$

(Since the above series converges uniformly on relatively compact subsets of $\tilde{V}_{0}$, $F^{\prime} \in \mathcal{O}\left(V^{\prime} \cup V^{\prime \prime}, M_{k}(\mathbb{C})\right.$ and satisfies the required condition.)

Now, we set $H^{\prime}:=\exp \left(F^{\prime}\right) \in \mathcal{O}\left(V^{\prime} \cup V^{\prime \prime}, G L_{k}(\mathbb{C})\right)$ completing the proof of the lemma in this case.

Further, let $H \in \mathcal{O}\left(U^{\prime} \cap U^{\prime \prime}, G L_{k}(\mathbb{C})\right)$ be an arbitrary $G L_{k}(\mathbb{C})$-valued bounded holomorphic map belonging to the connected component of the identity map $I$ of $\mathcal{O}\left(U^{\prime} \cap U^{\prime \prime}, G L_{k}(\mathbb{C})\right)$.

Let us show that $\left.H\right|_{V^{\prime} \cap V^{\prime \prime}}$ can be presented in the form

$$
\left.H\right|_{V^{\prime} \cap V^{\prime \prime}}=H^{1} \cdots H^{l}
$$

where each $H^{i} \in \mathcal{O}\left(V^{\prime} \cap V^{\prime \prime}, G L_{k}(\mathbb{C})\right), 1 \leq i \leq l$, satisfies

$$
\left\|I-H^{i}\right\|_{V^{\prime} \cap V^{\prime \prime}}<\frac{1}{2}
$$

In fact, since $H$ belongs to the connected component of the identity map $I$, there exists a continuous path $H_{t} \in \mathcal{O}\left(U^{\prime} \cap U^{\prime \prime}, G L_{k}(\mathbb{C})\right)(t \in[0,1])$ such that $H_{0}=I$ 
and $H_{1}=H$. Consider a partition $0=t_{0}<t_{1}<\cdots<t_{l}=1$ of the unit interval $[0,1]$, and define

$$
H^{i}(z, \omega)=H_{t_{i-1}}^{-1}(z, \omega) H_{t_{i}}(z, \omega), \quad(z, \omega) \in V^{\prime} \cap V^{\prime \prime}, \quad 1 \leq i \leq l,
$$

which gives us identity (7.37). Provided that $\max _{1 \leq i \leq l-1}\left|t_{i+1}-t_{i}\right|$ is sufficiently small, inequality (7.38) holds for all $1 \leq i \leq l$.

Now, according to the first case there exist $\left(H^{i}\right)^{\prime} \in \mathcal{O}\left(V^{\prime} \cup V^{\prime \prime}, G L_{k}(\mathbb{C})\right)$ such that $\left.\left(H^{i}\right)^{\prime}\right|_{V^{\prime} \cap V^{\prime \prime}}=\left.H^{i}\right|_{V^{\prime} \cap V^{\prime \prime}}$. We define $H^{\prime}:=\left(H^{1}\right)^{\prime} \cdots\left(H^{l}\right)^{\prime}$.

Corollary 7.18. In the notation of Lemma 7.17, for any open polydisk $V_{0} \Subset U_{0}$ and open subsets $L^{\prime} \Subset K^{\prime}, L^{\prime \prime} \Subset K^{\prime \prime}$ there exist functions $h^{\prime} \in \mathcal{O}\left(V^{\prime}, G L_{k}(\mathbb{C})\right)$, $h^{\prime \prime} \in \mathcal{O}\left(V^{\prime \prime}, G L_{k}(\mathbb{C})\right)$ such that

$$
H=h^{\prime} h^{\prime \prime} \quad \text { on } V^{\prime} \cap V^{\prime \prime} .
$$

Proof. Let $H^{\prime} \in \mathcal{O}\left(V^{\prime} \cup V^{\prime \prime}, G L_{k}(\mathbb{C})\right)$ be as in Lemma 7.17. Since $\left.H^{\prime}\right|_{V^{\prime} \cap V^{\prime \prime}}=$ $\left.H\right|_{V^{\prime} \cap V^{\prime \prime}}$, we can choose $h^{\prime}:=\left.H^{\prime}\right|_{V^{\prime}}, h^{\prime \prime}:=I$.

Lemma 7.19. Any analytic homomorphism $\varphi:\left.\left.\mathcal{O}\right|_{U} ^{k} \rightarrow \mathcal{O}\right|_{U} ^{l}$ is determined by a holomorphic function $\Phi \in \mathcal{O}\left(U, M_{l \times k}(\mathbb{C})\right)$.

The proof of Lemma 7.19 follows directly from the definitions (cf. Section 4.4).

We extend to our framework the notion of a completely exact sequence of sheaves from [31]:

Definition 7.20. Let $\mathcal{R}, \mathcal{B}_{i}, 1 \leq i \leq N$, be analytic sheaves over $U$. We say that a sequence

$$
\mathcal{B}_{N} \longrightarrow \cdots \rightarrow \mathcal{B}_{2} \longrightarrow \mathcal{B}_{1} \rightarrow \mathcal{R} \longrightarrow 0
$$

is completely exact if for any $m \geq 1$ the sequence of sections

$$
\begin{aligned}
\Gamma\left(U, \operatorname{Hom}_{\mathcal{O}}\left(\mathcal{O}^{m}, \mathcal{B}_{N}\right)\right) & \longrightarrow \cdots \\
& \longrightarrow \Gamma\left(U, \operatorname{Hom}_{\mathcal{O}}\left(\mathcal{O}^{m}, \mathcal{B}_{1}\right)\right) \longrightarrow \Gamma\left(U, \operatorname{Hom}_{\mathcal{O}}\left(\mathcal{O}^{m}, \mathcal{R}\right)\right) \longrightarrow 0
\end{aligned}
$$

or, equivalently,

$$
\Gamma\left(U, \mathcal{B}_{N}^{m}\right) \longrightarrow \cdots \rightarrow \Gamma\left(U, \mathcal{B}_{1}^{m}\right) \longrightarrow \Gamma\left(U, \mathcal{R}^{m}\right) \longrightarrow 0
$$

is exact.

Here $\mathcal{B}_{i}^{m}$ and $\mathcal{R}^{m}$ stand for the direct product of $m$ copies of $\mathcal{B}_{i}$ and $\mathcal{R}$, respectively, and $\operatorname{Hom}_{\mathcal{O}}\left(\mathcal{O}^{m}, \mathcal{B}_{i}\right), \operatorname{Hom}_{\mathcal{O}}\left(\mathcal{O}^{m}, \mathcal{R}\right)$ are the sheaves of germs of analytic homomorphisms $\mathcal{O}^{m} \rightarrow \mathcal{B}_{i}, \mathcal{O}^{m} \rightarrow \mathcal{R}$, respectively.

Note that if sequence (7.40) is exact for $m=1$, then it is exact for all $m \geq 1$.

Lemma 7.21. Let $\mathcal{B}, \mathcal{C}$ be analytic sheaves on $U$. If sequence $\mathcal{B} \stackrel{\gamma}{\rightarrow} \mathcal{C} \rightarrow 0$ is completely exact, and $\varphi:\left.\mathcal{O}^{k}\right|_{U} \rightarrow \mathcal{C}$ is an analytic homomorphism, then there is an analytic homomorphism $\psi:\left.\mathcal{O}^{k}\right|_{U} \rightarrow \mathcal{B}$ such that $\varphi=\gamma \psi$. 
Proof. We can take $\psi$ in the preimage of $\varphi$ under the surjective homomorphism

$$
\bar{\gamma}: \Gamma\left(U, \operatorname{Hom}_{\mathcal{O}}\left(\mathcal{O}^{k}, \mathcal{B}\right)\right) \rightarrow \Gamma\left(U, \operatorname{Hom}_{\mathcal{O}}\left(\mathcal{O}^{k}, \mathcal{C}\right)\right)
$$

induced by $\gamma$ (see Definition 7.20).

Lemma 7.22 (Three lemma). Let $\mathcal{A}, \mathcal{B}$ and $\mathcal{C}$ be analytic sheaves on $U$. Suppose that sequence

$$
0 \rightarrow \mathcal{A} \stackrel{\beta}{\rightarrow} \mathcal{B} \stackrel{\gamma}{\rightarrow} \mathcal{C} \longrightarrow 0
$$

is completely exact. If two sheaves among $\mathcal{A}, \mathcal{B}$ and $\mathcal{C}$ have free resolutions of length $N+n$, where $n:=\operatorname{dim}_{\mathbb{C}} U_{0}, N \geq n+2$, then the third one has a free resolution of length $N-n-1$.

The proof of Lemma 7.22 follows closely the arguments in the proofs of an analogous result (for "cohesive sheaves") in [31]. For the sake of completeness, we provide the proof of Lemma 7.22 in the Appendix; but now we will use it in the proof of Lemma 7.16 .

Proof of Lemma 7.16. We denote $U_{1}:=U_{0} \times K_{1}, U_{2}:=U_{0} \times K_{2}$. Let $N \geq n+1$. Consider free resolutions of $\mathcal{R}$ of length $M \geq 4 N$,

$$
\left.\left.\left.\mathcal{O}^{k_{M, i}}\right|_{U_{i}} \longrightarrow \cdots \longrightarrow \mathcal{O}^{k_{1, i}}\right|_{U_{i}} \stackrel{\alpha_{i}}{\longrightarrow} \mathcal{R}\right|_{U_{i}} \longrightarrow 0, \quad i=1,2 .
$$

Consider the end portions of (7.41):

$$
\left.\left.\mathcal{O}^{k_{i}}\right|_{U_{i}} \stackrel{\alpha_{i}}{\longrightarrow} \mathcal{R}\right|_{U_{i}} \longrightarrow 0, \quad i=1,2 .
$$

Let $U:=U_{0} \times\left(K_{1} \cup K_{2}\right)$. We denote by $\pi_{i}:\left.\left.\left.\mathcal{O}^{k_{1}}\right|_{U} \oplus \mathcal{O}^{k_{2}}\right|_{U} \rightarrow \mathcal{O}^{k_{i}}\right|_{U}, i=1,2$, the natural projection homomorphisms.

First, let us show that there exists an injective analytic homomorphism $H$ : $\left.\left.\left.\left.\mathcal{O}^{k_{1}}\right|_{U} \oplus \mathcal{O}^{k_{2}}\right|_{U} \rightarrow \mathcal{O}^{k_{1}}\right|_{U} \oplus \mathcal{O}^{k_{2}}\right|_{U}$ such that $\alpha_{1} \pi_{1} H=\alpha_{2} \pi_{2}$. By Proposition $7.1(1)$ sequence (7.41) truncated to the $N$-th term (and, hence, sequence (7.42)) is completely exact. By Lemma 7.21 we can factor $\alpha_{1}=\alpha_{2} \psi, \alpha_{2}=\alpha_{1} \varphi$ on $U_{1} \cap U_{2}$ for some analytic homomorphisms $\psi:\left.\left.\mathcal{O}^{k_{1}}\right|_{U_{1} \cap U_{2}} \rightarrow \mathcal{O}^{k_{2}}\right|_{U_{1} \cap U_{2}}, \varphi:\left.\mathcal{O}^{k_{2}}\right|_{U_{1} \cap U_{2}} \rightarrow$ $\left.\mathcal{O}^{k_{1}}\right|_{U_{1} \cap U_{2}}$. Now, identifying sheaf homomorphisms $\psi, \varphi$ with the holomorphic matrix functions that determine them (see Lemma 7.19), we define

$$
H=\left(\begin{array}{cc}
I_{k_{1}} & \varphi \\
0 & I_{k_{2}}
\end{array}\right)\left(\begin{array}{cc}
I_{k_{1}} & 0 \\
\psi & I_{k_{2}}
\end{array}\right)^{-1} \in \mathcal{O}\left(U_{1} \cap U_{2}, G L_{k}(\mathbb{C})\right),
$$

where $k:=k_{1}+k_{2}$. It is immediate that $\alpha_{1} \pi_{1} H=\alpha_{2} \pi_{2}$. The map $H$ belongs to the connected component of the identity map in $\mathcal{O}\left(U_{1} \cap U_{2}, G L_{k}(\mathbb{C})\right)$. Indeed, consider a path $H_{t} \in \mathcal{O}\left(U_{1} \cap U_{2}, G L_{k}(\mathbb{C})\right)(t \in[0,1])$,

$$
H_{t}:=\left(\begin{array}{cc}
I_{k_{1}} & t \varphi \\
0 & I_{k_{2}}
\end{array}\right)\left(\begin{array}{cc}
I_{k_{1}} & 0 \\
t \psi & I_{k_{2}}
\end{array}\right)^{-1}
$$

so that $H_{0}=I_{k}, H_{1}=H$. 
Next, let $L_{i} \Subset K_{i}, L_{i} \in \mathfrak{Q}(i=1,2)$ and $V_{0} \Subset U_{0}$ be an open polydisk, and $x_{0} \in V_{0}$. Let $L_{i}^{m} \in \mathfrak{Q}(i=1,2), m \geq 1$, be the collection of open subsets of $K_{i}$ such that $L_{i} \Subset L_{i}^{m+1} \Subset L_{i}^{m} \Subset K_{i}$ for all $m \geq 1(i=1,2)$ obtained in Lemma $5.3(3)$.

Let $\left\{V_{0}^{m}\right\}$ be a collection of open polydisks such that $V_{0} \Subset V_{0}^{m+1} \Subset V_{0}^{m} \Subset U_{0}$ for all $m \geq 1$.

We set $V_{i}^{m}:=V_{0}^{m} \times L_{i}^{m}, V_{i}:=V_{0} \times L_{i}(i=1,2), m \geq 1$.

We now amalgamate the free resolutions of $\mathcal{R}$ over $V_{1} \cup V_{2}$.

Let $m=1$. By Corollary 7.18 there exist functions $h_{i} \in \mathcal{O}\left(V_{i}^{1}, G L_{k}(\mathbb{C})\right)$ $(i=1,2)$ such that $H=h_{1} h_{2}$ on $V_{1}^{1} \cap V_{2}^{1}$. Since $\alpha_{1} \pi_{1} H=\alpha_{2} \pi_{2}$, the sheaf homomorphisms

$$
\begin{gathered}
\alpha_{1} \pi_{1} h_{1}:\left.\left.\left.\mathcal{O}^{k_{1}}\right|_{V_{1}^{1}} \oplus \mathcal{O}^{k_{2}}\right|_{V_{1}^{1}} \longrightarrow \mathcal{R}\right|_{V_{1}^{1}} \longrightarrow 0, \\
\alpha_{2} \pi_{2} h_{2}^{-1}:\left.\left.\left.\mathcal{O}^{k_{1}}\right|_{V_{2}^{1}} \oplus \mathcal{O}^{k_{2}}\right|_{V_{2}^{1}} \longrightarrow \mathcal{R}\right|_{V_{2}^{1}} \longrightarrow 0
\end{gathered}
$$

coincide over $V_{1}^{1} \cap V_{2}^{1}$; they induce an analytic homomorphism

$$
\alpha:\left.\left.\left.\mathcal{O}^{k_{1}}\right|_{V_{1}^{1} \cup V_{2}^{1}} \oplus \mathcal{O}^{k_{2}}\right|_{V_{1}^{1} \cup V_{2}^{1}} \longrightarrow \mathcal{R}\right|_{V_{1}^{1} \cup V_{2}^{1}}
$$

Let $\mathcal{R}_{1}:=\operatorname{Ker} \alpha$. The sequence

$$
\left.\left.\left.\left.0 \longrightarrow \mathcal{R}_{1}\right|_{V_{1}^{1} \cup V_{2}^{1}} \longrightarrow \mathcal{O}^{k_{1}}\right|_{V_{1}^{1} \cup V_{2}^{1}} \oplus \mathcal{O}^{k_{2}}\right|_{V_{1}^{1} \cup V_{2}^{1}} \stackrel{\alpha}{\longrightarrow} \mathcal{R}\right|_{V_{1}^{1} \cup V_{2}^{1}} \longrightarrow 0
$$

is completely exact over sets $V_{1}^{1}$ and $V_{2}^{1}$ since sequences (7.42) are. By Lemma 7.22 the analytic sheaf $\mathcal{R}_{1}$ has free resolutions over $V_{1}^{1}$ and $V_{2}^{1}$ (of length $4 N-2 n-1$ ) because two other sheaves have.

Provided that $M$ was chosen sufficiently large, we can repeat this construction $N-1$ times over subsets $V_{1}^{m}, V_{2}^{m}, 1 \leq m \leq N-1$, obtaining in the end a free resolution of $\mathcal{R}$ over $V_{1} \cup V_{2}$ having length $N$. Since $V_{0}, L_{1}, L_{2}$ and $N$ were arbitrary, the required result follows.

\section{Proof of Theorem 4.6}

We have to prove that for a connected complex manifold $M$ and a map $F \in$ $\mathcal{O}\left(M, c_{\mathfrak{a}} X\right)$ there exists a 'horizontal layer' $\hat{X}_{H}(H \in \Upsilon)$ such that $F(M) \subset \hat{X}_{H}$ (see subsection 4.1 for notation).

Proof. Let $y_{0}:=F\left(z_{0}\right)$ for some $z_{0} \in M$. Then $y_{0} \in \hat{X}_{H_{0}}$ for some $H_{0} \in \Upsilon$. Also, $y_{0}$ is contained in a coordinate chart $\hat{\Pi}\left(U_{0}, K\right) \subset c_{\mathfrak{a}} X$. In what follows, we identify $\hat{\Pi}\left(U_{0}, K\right)$ with $U_{0} \times K$, see subsection 4.2 , so that $y_{0}=\left(x_{0}, \eta_{0}\right)$ for some $x_{0} \in U_{0}, \eta_{0} \in K$. Let $\pi_{K}: U_{0} \times K \rightarrow K$ be the natural projection. Then for each $h \in C(K)$ the pullback $h_{K}:=\left(\pi_{K}\right)^{*} h \in \mathcal{O}\left(U_{0}, K\right)$ and is constant on subsets $U_{0} \times\{\eta\}$ for all $\eta \in K$. Since $F$ is a holomorphic map, $F^{*} h_{K}$ is holomorphic on open subset $F^{-1}\left(U_{0} \times K\right) \subset M$. Since the complex conjugate $\bar{h}_{K}$ of $h_{K}$ also belongs to $\mathcal{O}\left(U_{0}, K\right)$, the function $F^{*} \bar{h}_{K}=\bar{F}^{*} h_{K}$ is holomorphic on $F^{-1}\left(U_{0} \times K\right)$ as well. Therefore, $F^{*} h_{K}$ must be locally constant. Let $W \subset M$ be the connected component of $F^{-1}\left(U_{0} \times K\right)$ containing $z_{0}$; then $F^{*} h_{K} \equiv h\left(\eta_{0}\right)$ on $W$. 
Now, let us show that $F(W) \subset U_{0} \times\left\{\eta_{0}\right\} \subset \hat{X}_{H_{0}}$. Indeed, there exist open subsets $L_{\lambda} \subset K(\lambda \in \Lambda)$ such that $\eta_{0} \in L_{\lambda}$ for all $\lambda$ and $\cap_{\lambda \in \Lambda} L_{\lambda}=\left\{\eta_{0}\right\}$. Since $\hat{G}_{\mathfrak{a}}$ is a compact space and each subset $L_{\lambda}$ is open in $\hat{G}_{\mathfrak{a}}$, for every $\lambda$ there exists a continuous partition of unity subordinate to the open cover $\left\{L_{\lambda}, \hat{G}_{\mathfrak{a}} \backslash\left\{\eta_{0}\right\}\right\}$ of $\hat{G}_{\mathfrak{a}}$. We denote by $h_{\lambda} \in C(K)$ the restriction to $K$ of the element of the partition of unity with support in $L_{\lambda}$. Then $0 \leq h_{\lambda} \leq 1, h_{\lambda}\left(\eta_{0}\right)=1, h_{\lambda}(\eta)=0$ on $K \backslash L_{\lambda}$. Since $F^{*}\left(h_{\lambda}\right)_{K} \equiv h_{\lambda}\left(\eta_{0}\right)=1$ for all $\lambda$, we obtain that $F(W) \subset U_{0} \times L_{\lambda}$ for all $\lambda$; hence, $F(W) \subset U_{0} \times \cap_{\lambda \in \Lambda} L_{\lambda}=U_{0} \times\left\{\eta_{0}\right\} \subset \hat{X}_{H_{0}}$.

We have established that every point in $M$ has a neighbourhood $W$ such that $F(W) \subset \hat{X}_{H}$ for some $H \in \Upsilon$. Since $\hat{X}_{H_{1}} \cap \hat{X}_{H_{2}}=\varnothing$ if $H_{1} \neq H_{2}$ and $M$ is connected, the latter implies that $F(M) \subset \hat{X}_{H_{0}}$ for a certain $H_{0}$ and completes the proof of the theorem.

\subsection{Proof of Proposition 4.7}

The proof of Proposition 4.7 essentially repeats the proof of an analogous result for coherent analytic sheaves on complex manifolds, see, e.g., [24].

First, let $\mathcal{A}$ be a coherent subsheaf of $\mathcal{O}^{k}$ and let $U \in \mathfrak{B}$ (see (4.4)). By Lemma $5.3(2)$ there exist open sets $V_{k} \in \mathfrak{B}$ such that $V_{k} \Subset V_{k+1} \Subset U$ for all $k$, and $U=\cup_{k} V_{k}$. We endow space $\Gamma(U, \mathcal{A})$ of sections of sheaf $\mathcal{A}$ over $U$ with the topology of uniform convergence on $\bar{V}_{k}$ for all $k$. Then $\Gamma(U, \mathcal{A})$ becomes a metrizable topological vector space. We have to show that space $\Gamma(U, \mathcal{A})$ is complete, i.e., it is a Fréchet space.

It is easy to see that space $\Gamma\left(U, \mathcal{O}^{k}\right)$ endowed with such topology is complete. Since $\mathcal{A}$ is coherent, we may assume that there exists a free resolution (2.4) of $\mathcal{A}$ over $U$ of length $4 N, N>n:=\operatorname{dim}_{\mathbb{C}} X_{0}$. Therefore, we have a short exact sequence

$$
\left.\left.0 \longrightarrow \operatorname{Ker} \varphi \stackrel{\iota}{\longrightarrow} \mathcal{O}^{m}\right|_{U} \stackrel{\varphi}{\longrightarrow} \mathcal{A}\right|_{U} \longrightarrow 0,
$$

where $\iota$ denotes the inclusion. In the proof of Proposition 7.1(1) we have shown that the sequence of sections

$$
0 \longrightarrow \Gamma(U, \operatorname{Ker} \varphi) \stackrel{\bar{\iota}}{\longrightarrow} \Gamma\left(U, \mathcal{O}^{m}\right) \stackrel{\bar{\varphi}}{\longrightarrow} \Gamma(U, \mathcal{A}) \longrightarrow 0
$$

is exact (see Lemmas 7.13 and 7.15). By our assumption $\Gamma(U, \mathcal{A}) \subset \Gamma\left(U, \mathcal{O}^{k}\right)$. By Lemma 7.19 the $\Gamma(U, \mathcal{O})$-module homomorphism $\bar{\varphi}: \Gamma\left(U, \mathcal{O}^{m}\right) \rightarrow \Gamma\left(U, \mathcal{O}^{k}\right)$ is determined by a $k \times m$ matrix with entries in $\mathcal{O}(U)$, hence it is continuous; further, $\bar{\iota}$ is continuous. Since sequence $(8.1)$ is exact, $\Gamma(U, \operatorname{Ker} \varphi) \cong \operatorname{Ker} \bar{\varphi}$, hence $\Gamma(U, \operatorname{Ker} \varphi)$ is closed. Therefore, $\Gamma(U, \mathcal{A})$, being a quotient of a complete space by its closed subspace, is a complete space.

We note that by the open mapping theorem the topology on $\Gamma(U, \mathcal{A})$ coincides with the quotient topology determined by (8.1).

Now, let $\mathcal{A}$ be an arbitrary coherent sheaf on $c_{\mathfrak{a}} X$. Similarly, we have a free resolution (2.4) of $\mathcal{A}$ over a neighbourhood $U$ of length $4 N, N>n$, which yields a short exact sequence of sheaves

$$
\left.\left.0 \longrightarrow \operatorname{Ker} \varphi \stackrel{\iota}{\longrightarrow} \mathcal{O}^{m}\right|_{U} \stackrel{\varphi}{\longrightarrow} \mathcal{A}\right|_{U} \longrightarrow 0
$$


and an exact sequence of sections

$$
0 \longrightarrow \Gamma(U, \operatorname{Ker} \varphi) \stackrel{\bar{\iota}}{\longrightarrow} \Gamma\left(U, \mathcal{O}^{m}\right) \stackrel{\bar{\varphi}}{\rightarrow} \Gamma(U, \mathcal{A}) \longrightarrow 0 .
$$

Using Lemma 7.22 (Three lemma), we obtain that Ker $\varphi$ is a coherent subsheaf of $\left.\mathcal{O}^{m}\right|_{U}$, so by the previous part the subspace $\Gamma(U, \operatorname{Ker} \varphi) \subset \Gamma\left(U, \mathcal{O}^{m}\right)$ is closed. We introduce in $\Gamma(U, \mathcal{A})$ the quotient topology defined by $(8.3)$ which makes it a complete (i.e., Fréchet) space and also implies the last assertion of the proposition concerning the family of semi-norms determining the topology in $\Gamma(U, \mathcal{A})$.

Let us show that thus defined topology on $\Gamma(U, \mathcal{A})$ does not depend on the choice of resolution (8.2). Suppose that there is another resolution

$$
\left.\left.0 \longrightarrow \operatorname{Ker} \varphi^{\prime} \stackrel{\iota}{\longrightarrow} \mathcal{O}^{m^{\prime}}\right|_{U} \stackrel{\varphi^{\prime}}{\longrightarrow} \mathcal{A}\right|_{U} \longrightarrow 0 .
$$

By Lemma 9.1 there is a homomorphism $\psi:\left.\left.\mathcal{O}^{m}\right|_{U} \rightarrow \mathcal{O}^{m^{\prime}}\right|_{U}$ such that the diagram of exact sequences of sheaves

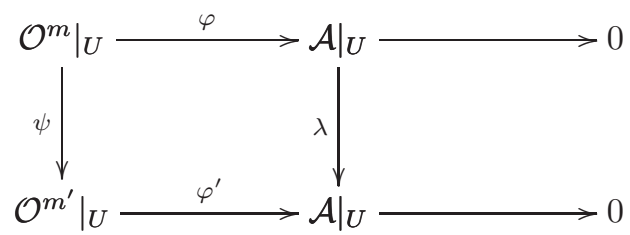

is commutative. Therefore, we have a commutative diagram

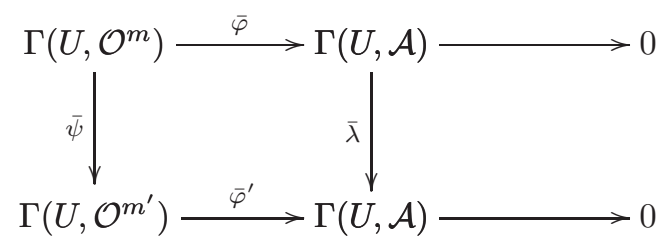

of exact sequences of sections. By our construction $\bar{\varphi}, \bar{\varphi}^{\prime}$ are continuous and surjective, $\bar{\psi}$ is continuous as a homomorphism of sections of free sheaves. By the open mapping theorem the preimage of an open set by $\bar{\lambda}^{-1}=\bar{\varphi} \circ(\bar{\psi})^{-1} \circ\left(\bar{\varphi}^{\prime}\right)^{-1}$ is open, so $\bar{\lambda}$ is continuous and, hence, it is a homeomorphism.

Finally, let $\gamma: \mathcal{A} \rightarrow \mathcal{B}$ be an analytic homomorphism. Let us show that $\gamma$ is continuous. Analogously to the previous part applying Lemma 9.1 we obtain a commutative diagram of exact sequences of sheaves which yields a commutative diagram of exact sequences

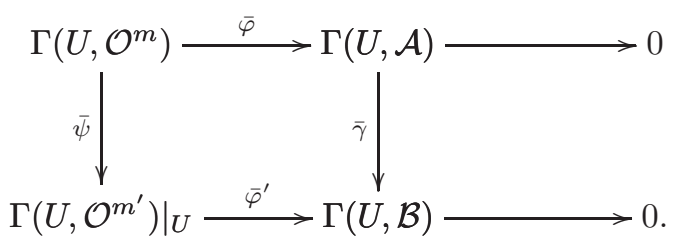

As before, the continuity of $\bar{\gamma}$ can be deduced from the continuity of the other homomorphisms in the diagram. This completes the proof of the proposition. 


\section{Appendix}

\subsection{Proof of Lemma 7.22}

The proof follows closely the arguments in [31]. We will need the following lemmas.

Lemma 9.1. Let $\mathcal{A}$ be an analytic sheaf on $U$ that admits a free resolution of length $N$

$$
\left.\left.\left.\mathcal{F}_{N}\right|_{U} \stackrel{\varphi_{N-1}}{\longrightarrow} \cdots \stackrel{\varphi_{2}}{\longrightarrow} \mathcal{F}_{2}\right|_{U} \stackrel{\varphi_{1}}{\longrightarrow} \mathcal{F}_{1}\right|_{U} \stackrel{\varphi_{0}}{\longrightarrow} \mathcal{A} \longrightarrow 0 .
$$

Given a completely exact sequence of analytic sheaves $\mathcal{B}_{i}$ on $U, 0 \leq i \leq N$,

$$
\mathcal{B}_{N} \stackrel{\beta_{N-1}}{\longrightarrow} \cdots \rightarrow \mathcal{B}_{2} \stackrel{\beta_{1}}{\longrightarrow} \mathcal{B}_{1} \stackrel{\beta_{0}}{\longrightarrow} \mathcal{B}_{0} \longrightarrow 0
$$

a sheaf homomorphism $\Phi_{0}: \mathcal{A} \rightarrow \mathcal{B}_{0}$ can be extended to a homomorphism $\Phi_{j}:\left.\mathcal{F}_{j}\right|_{U}$ $\rightarrow \mathcal{B}_{j}(0 \leq j \leq N)$ of sequences (9.1) and (9.2).

Proof. The proof is by induction. We put $\varphi_{-1}:=0, \beta_{-1}:=0$. Suppose that for $0 \leq j \leq r, r \leq N-1$ the homomorphisms $\Phi_{j}:\left.\mathcal{F}_{j}\right|_{U} \rightarrow \mathcal{B}_{j}$ have been constructed, so that $\Phi_{j-1} \varphi_{j-1}=\beta_{j-1} \Phi_{j}$. If $r=N-1$, then we are done. For $r<N-1$ we have $\beta_{r-1}\left(\Phi_{r} \varphi_{r}\right)=\Phi_{r-1} \varphi_{r-1} \varphi_{r}=0$. The sequence

$$
\Gamma\left(U, \operatorname{Hom}_{\mathcal{O}}\left(\mathcal{F}_{r+1}, \mathcal{B}_{r+1}\right)\right) \longrightarrow \cdots \rightarrow \Gamma\left(U, \operatorname{Hom}_{\mathcal{O}}\left(\mathcal{F}_{r+1}, \mathcal{B}_{0}\right)\right) \longrightarrow 0
$$

is exact since (9.2) is completely exact (see Definition 7.20), hence there is a homomorphism $\Phi_{r+1} \in \Gamma\left(U, \operatorname{Hom}_{\mathcal{O}}\left(\mathcal{F}_{r+1}, \mathcal{B}_{r+1}\right)\right)$ such that $\Phi_{r} \varphi_{r}=\beta_{r} \Phi_{r+1}$ over $U$, as required.

Lemma 9.2. Given a free resolution (9.1) of an analytic sheaf $\mathcal{A}$ on $U$ of length $N$ the sheaf $\operatorname{Ker} \varphi_{n-1}=\operatorname{Im} \varphi_{n}$ on $U, 1 \leq n \leq N-1$, has a free resolution of length $N-n$.

Proof. Follows immediately from Definition 7.12 of a free resolution of an analytic sheaf.

Lemma 9.3. Let $\mathcal{A}_{0}$ be an analytic sheaf over $U$. Suppose that for a given $N \geq 1$ there exists a completely exact sequence of analytic sheaves $\mathcal{A}_{i}$ on $U, 1 \leq i \leq$ $2 N+2$,

$$
\mathcal{A}_{2 N+2} \stackrel{\alpha_{M-1}}{\longrightarrow} \cdots \stackrel{\alpha_{1}}{\longrightarrow} \mathcal{A}_{1} \stackrel{\alpha_{0}}{\longrightarrow} \mathcal{A}_{0} \longrightarrow 0
$$

such that sheaves $\mathcal{A}_{i}, 1 \leq i \leq 2 N+2$, have free resolutions of length $n+N$, where $n:=\operatorname{dim}_{\mathbb{C}} U_{0}$. Then $\mathcal{A}_{0}$ has a free resolution of length $N$.

Proof. Let $M:=2 N+2$.

(1) First, we construct a completely exact sequence of length $M-2$ of the form

$$
\mathcal{B}_{M-2} \stackrel{\beta_{M-3}}{\longrightarrow} \cdots \stackrel{\beta_{2}}{\longrightarrow} \mathcal{B}_{2} \stackrel{\beta_{1}}{\longrightarrow} \mathcal{B}_{1} \stackrel{\varepsilon_{0}}{\longrightarrow} \mathcal{A}_{0} \longrightarrow 0
$$


where $\mathcal{B}_{1}=\left.\mathcal{O}^{k}\right|_{U}$ for some $k \geq 0$ is a free sheaf and $\mathcal{B}_{k}, 2 \leq k \leq M-2$, are analytic sheaves on $U$ having free resolutions of length $N+n-1$. Let

$$
\mathcal{F}_{n+N, k} \longrightarrow \cdots \rightarrow \mathcal{F}_{1, k} \stackrel{\omega_{k}}{\longrightarrow} \mathcal{A}_{k} \longrightarrow 0
$$

be a free resolution of $\mathcal{A}_{k}, 1 \leq k \leq M$. By Lemma 9.1 there exist analytic homomorphisms $\psi_{k}$ such that the diagram

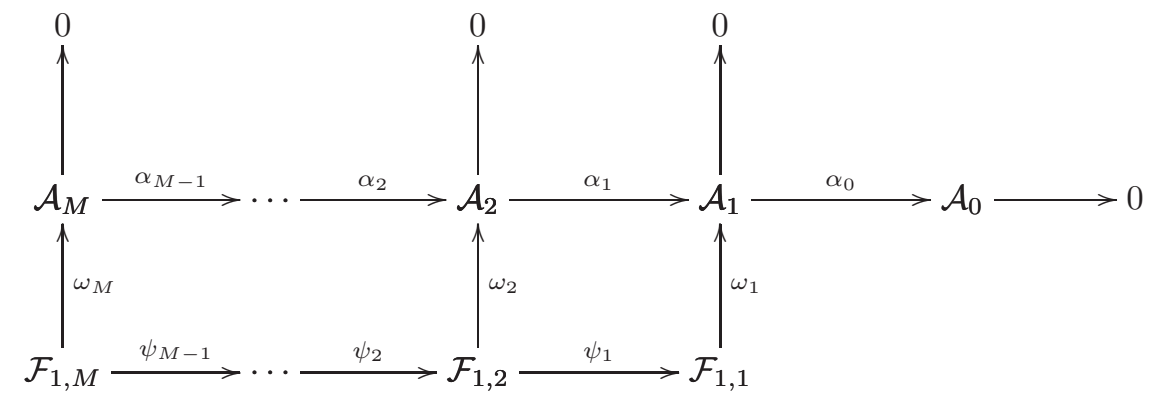

is commutative. Let us show that the sequence

(9.6) $\quad \mathcal{F}_{1, M} \oplus \operatorname{Ker} \omega_{M-1} \stackrel{\beta_{M-1}}{\longrightarrow} \cdots \stackrel{\beta_{2}}{\longrightarrow} \mathcal{F}_{1,2} \oplus \operatorname{Ker} \omega_{1} \stackrel{\beta_{1}}{\longrightarrow} \mathcal{F}_{1,1} \stackrel{\beta_{0}}{\longrightarrow} \mathcal{A}_{0} \longrightarrow 0$

truncated to term $\mathcal{F}_{1, M-2} \oplus \operatorname{Ker} \omega_{M-3}$ is completely exact. Here $\beta_{0}:=\alpha_{0} \omega_{1}, \beta_{1}:=$ $\psi_{1}-\iota_{1}$, where $\iota_{k}: \operatorname{Ker} \omega_{k} \hookrightarrow \mathcal{F}_{1, k}$ is an inclusion, and $\beta_{k}=\left(\iota_{k} \oplus \psi_{k-1}\right)\left(\psi_{k}-\iota_{k}\right)$, for $k \geq 2$.

Indeed, we apply to $(9.5)$ and (9.6) left exact functor $\Gamma\left(U, \operatorname{Hom}_{\mathcal{O}}(\mathcal{E}, \cdot)\right)$, where $\mathcal{E}$ is a free sheaf. Let

$$
\begin{aligned}
& A_{k}:=\Gamma\left(U, \operatorname{Hom}_{\mathcal{O}}\left(\mathcal{E}, \mathcal{A}_{k}\right)\right), \quad(0 \leq k \leq M), \\
& F_{k}:=\Gamma\left(U, \operatorname{Hom}_{\mathcal{O}}\left(\mathcal{E}, \mathcal{F}_{1, k}\right)\right), \quad(1 \leq k \leq M) .
\end{aligned}
$$

Then we obtain commutative diagrams of abelian groups

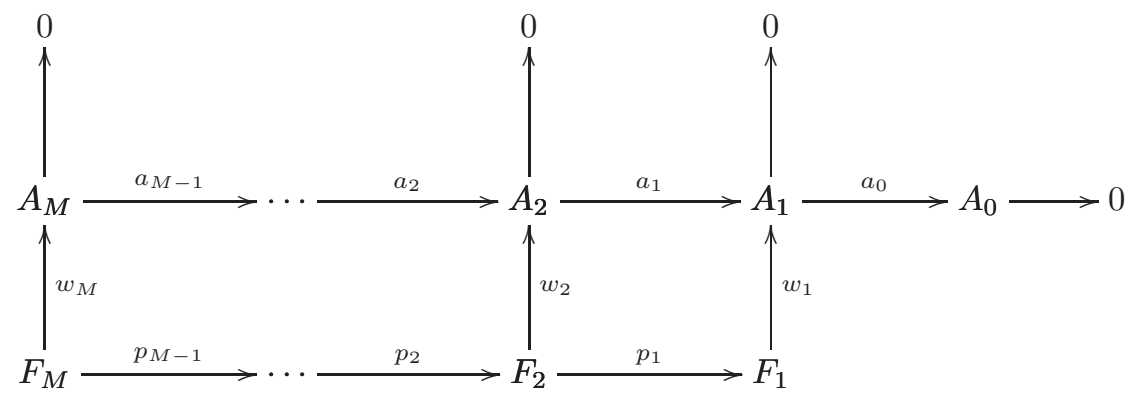

and

$$
F_{M} \oplus \operatorname{Ker} w_{M-1} \stackrel{b_{M-1}}{\longrightarrow} \cdots \stackrel{b_{2}}{\longrightarrow} F_{2} \oplus \operatorname{Ker} w_{1} \stackrel{b_{1}}{\longrightarrow} F_{1} \stackrel{b_{0}}{\longrightarrow} A_{0} \longrightarrow 0
$$


Note that complete exactness of sequence (9.6) truncated to $\mathcal{F}_{1, M-2} \oplus \operatorname{Ker} \omega_{M-3}$ is equivalent by definition to the exactness of sequence (9.8) truncated to term $F_{M-2} \oplus$ Ker $w_{M-3}$. By Definition 7.20 the middle row of (9.7) is exact. Also, by Proposition 7.1 (1) each $w_{k}, 1 \leq k \leq M$, is surjective, so the columns of (9.7) are exact. Hence, we have analogous identities

$$
b_{0}=a_{0} w_{1}, \quad b_{1}=p_{1}-i_{1}, \quad b_{k}=\left(i_{k} \oplus p_{k-1}\right)\left(p_{k}-i_{k}\right),
$$

where $i_{k}$ : Ker $w_{k} \hookrightarrow F_{k}$ is an inclusion. Let us show that (9.8) is exact up to term $F_{M-2} \oplus$ Ker $w_{M-3}$. First, note that $b_{0}$ is surjective because both $a_{0}$ and $w_{1}$ are. Second, if $\xi \in \operatorname{Ker} b_{0}$, then $w_{1}(\xi) \in \operatorname{Ker} a_{0}=\operatorname{Im} a_{1}=\operatorname{Im} a_{1} w_{2}=\operatorname{Im} w_{1} p_{1}$. Here we have used the fact that $w_{2}$ is surjective. Let $w_{1}(\xi)=w_{1}\left(p_{1}(\zeta)\right)$ and $\tau:=p_{1}(\zeta)-\xi \in \operatorname{Ker} w_{1}$, so that $\xi=b_{1}(\zeta, \tau) \in \operatorname{Im} b_{1}$. Third, if $1 \leq k \leq M-3$, and $(\xi, \eta) \in \operatorname{Ker} b_{k}=\operatorname{Ker}\left(p_{k}-i_{k}\right)$, then $\eta=p_{k}(\xi)$ and $0=w_{k}\left(p_{k}(\xi)\right)=a_{k}\left(w_{k+1}(\xi)\right)$; hence $w_{k+1}(\xi) \in \operatorname{Im} a_{k+1}=\operatorname{Im} a_{k+1} w_{k+2}=\operatorname{Im} w_{k+1} p_{k+1}$. Choose $\zeta$ so that $w_{k+1}(\xi)=w_{k+1}\left(p_{k+1}(\zeta)\right)$. Then $\tau:=p_{k+1}(\zeta)-\xi \in$ Ker $w_{k+1}$. We conclude that $(\xi, \eta)=b_{k+1}(\zeta, \tau) \in \operatorname{Im} b_{k+1}$, i.e., sequence (9.8) is indeed exact up to term $F_{M-2} \oplus \operatorname{Ker} w_{M-3}$.

Now, by Lemma 9.2 each $\mathcal{F}_{1, k} \oplus$ Ker $\omega_{k-1}$ has a free resolution of length $N+$ $n-1$. Hence, if we take

$$
\mathcal{B}_{1}:=\mathcal{F}_{1,1}, \quad \varepsilon_{0}:=\beta_{0}, \quad \mathcal{B}_{k}:=\mathcal{F}_{1, k} \oplus \operatorname{Ker} \omega_{k-1}, \quad 2 \leq k \leq M-2,
$$

we obtain the required completely exact sequence (9.4).

(2) Now, consider completely exact sequence obtained from (9.4),

$$
\mathcal{B}_{M} \stackrel{\beta_{M-1}}{\longrightarrow} \cdots \stackrel{\beta_{2}}{\rightarrow} \mathcal{B}_{2} \stackrel{\beta_{1}}{\longrightarrow} \text { Ker } \varepsilon_{0} \longrightarrow 0
$$

Applying case (1) to this sequence we obtain that there is a completely exact sequence

$$
\mathcal{D}_{M-4} \longrightarrow \cdots \rightarrow \mathcal{D}_{3} \longrightarrow \mathcal{D}_{2} \stackrel{\varepsilon_{1}}{\rightarrow} \text { Ker } \varepsilon_{0} \longrightarrow 0
$$

where $\mathcal{D}_{2}$ is a free sheaf, and each sheaf $\mathcal{D}_{k}, 3 \leq k \leq M-4$, has a free resolution of length $N+n-2$. Therefore, we have a completely exact sequence

$$
\mathcal{D}_{M-4} \longrightarrow \cdots \rightarrow \mathcal{D}_{3} \longrightarrow \mathcal{D}_{2} \stackrel{\varepsilon_{1}}{\rightarrow} \mathcal{B}_{1} \rightarrow \mathcal{A}_{0} \longrightarrow 0
$$

Continuing in this way (applying a similar argument to resolution of Ker $\varepsilon_{1}$, etc.) we finally obtain a free resolution of $\mathcal{A}_{0}$ of length $N$.

Proof of Lemma 7.22. We can assume that $\mathcal{A} \subset \mathcal{B}$ and that $\beta$ is the inclusion map.

(a) If $\mathcal{A}$ and $\mathcal{B}$ have free resolutions of length $N+n$, then Lemma 9.3 implies that $\mathcal{C}$ has a free resolution of length $N$ (and, in particular, of length $N-n-1$ ).

Consider two remaining cases. Sheaf $\mathcal{C}$ has a free resolution of length $N+n$,

$$
\mathcal{F}_{N+n} \longrightarrow \cdots \rightarrow \mathcal{F}_{1} \stackrel{\varphi}{\longrightarrow} \mathcal{C} \longrightarrow 0
$$


for some open $V_{0} \subset U_{0}$. By Proposition 7.1(1), the sequence (9.10) is completely exact. By Lemma 7.21 there is a commutative diagram

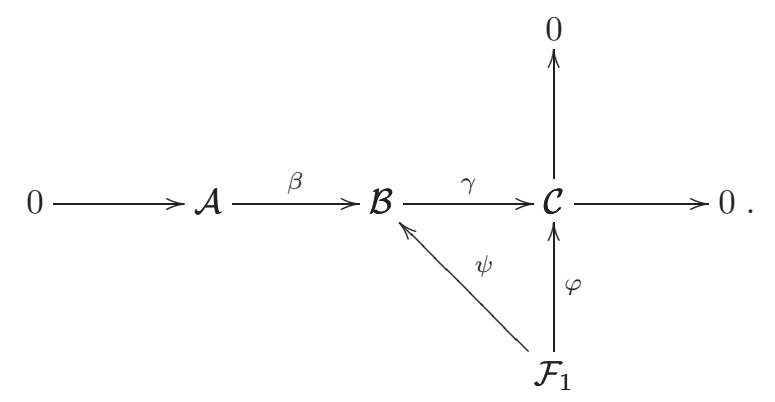

Let $\iota: \operatorname{Ker} \varphi \rightarrow \mathcal{F}_{1}$ denote the inclusion. Let us show that the sequence

$$
0 \longrightarrow \operatorname{Ker} \varphi \stackrel{\psi \oplus \iota}{\longrightarrow} \mathcal{A} \oplus \mathcal{F}_{1} \stackrel{\beta-\psi}{\longrightarrow} \mathcal{B} \longrightarrow 0
$$

is completely exact.

We apply functor $\Gamma\left(U, \operatorname{Hom}_{\mathcal{O}}(\mathcal{E}, \cdot)\right)$ to $(9.11)$ and $(9.12)$, where $\mathcal{E}$ is a free sheaf. We obtain diagrams of abelian groups

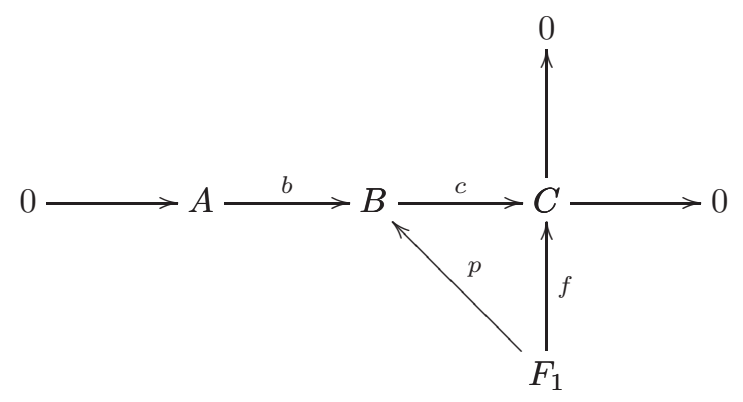

and

$$
0 \longrightarrow \text { Ker } f \stackrel{p \oplus i}{\longrightarrow} A \oplus F_{1} \stackrel{b-p}{\longrightarrow} B \longrightarrow 0 .
$$

The first diagram is commutative, its top row is exact (see Definition 7.20). By Proposition 7.1(1), we may assume that $f$ is surjective. The latter sequence is a complex and is exact at Ker $f$. We have to check that it is exact at the next two terms. If $(\xi, \eta) \in \operatorname{Ker}(b-p)$ then $p(\eta)=b(\xi)=\xi, 0=c(p(\eta))=f(\eta)$. Thus, $\eta \in \operatorname{Ker} f$ and $(\xi, \eta)=(p \oplus i)(\eta) \in \operatorname{Im}(p \oplus i)$, hence (9.13) is exact in the middle term. On the other hand, if $\zeta \in B$, then with some $\eta \in F$,

$$
-c(\zeta)=f(\eta)=c(p(\eta))
$$

i.e.,

$$
\zeta+p(\eta)=\xi \in \operatorname{Ker} c=A .
$$

Thus, $\zeta=\xi-p(\eta) \in \operatorname{Im}(b-p)$. We obtain that sequence (9.13) is exact, hence sequence (9.12) is completely exact.

Note that by Lemma $9.2 \operatorname{Ker} \varphi$ has a free resolution of length $N+n-1$. 
(b) The sheaf $\mathcal{A} \oplus \mathcal{F}_{1}$ has a free resolution of length $N+n-1$ over $U_{0} \times K$. By Lemma 9.3 sheaf $\mathcal{B}$ has a free resolution of length $N-1$ over $U_{0} \times K$ (in particular, of length $N-n-1)$.

(c) We may assume that $\mathcal{B}$ has a free resolution of length $N+n-1$ over $V_{0} \times K$. Since $\operatorname{Ker} \varphi$ has a free resolution of length $N+n-1$, by (b) $\mathcal{A} \oplus \mathcal{F}$ has a free resolution of length $N-1$. Since sequence $0 \rightarrow \mathcal{F} \rightarrow \mathcal{A} \oplus \mathcal{F} \rightarrow \mathcal{A} \rightarrow 0$ is completely exact as $\mathcal{F}$ is a free sheaf (see Corollary 7.10), we obtain by part (a) that $\mathcal{A}$ has a free resolution of length $N-n-1$.

\section{References}

[1] Bohr, H.: Almost periodic functions. Chelsea Publishing Co., New York, 1951.

[2] Brudnyi, A.: Projections in the space $H^{\infty}$ and the corona theorem for subdomains of coverings of finite bordered Riemann surfaces. Ark. Mat. 42 (2004), 31-59.

[3] Brudnyi, A.: On holomorphic functions of slow growth on coverings of strongly pseudoconvex manifolds. J. Funct. Anal. 249 (2007), 354-371.

[4] Brudnyi, A.: Hartogs type theorems on coverings of Stein manifolds. Internat. J. Math. 17 (2006), 339-349.

[5] BRUdnyi, A.: Representation of holomorphic functions on coverings of pseudoconvex domains in Stein manifolds via integral formulas on these domains. J. Funct. Anal. 231 (2006), 418-437.

[6] BRudnyi, A.: Holomorphic $L^{p}$-functions on coverings of strongly pseudoconvex manifolds. Proc. Amer. Math. Soc. 137 (2009), 227-234.

[7] Brudnyi, A.: Topology of the maximal ideal space of $H^{\infty}$. J. Funct. Anal. 189 (2002), 21-52.

[8] Brudnyi, A.: Contractibility of maximal ideal spaces of certain algebras of almost periodic functions. Integral Equations Operator Theory 52 (2005), 595-598.

[9] Brudnyi, A.: Banach-valued holomorphic functions on the maximal ideal space of $H^{\infty}$. Invent. Math. 193 (2013), 187-227.

[10] Brudnyi, A.: Holomorphic Banach vector bundles on the maximal ideal space of $H^{\infty}$ and the operator corona problem of Sz.-Nagy. Adv. Math. 232 (2013), $121-141$.

[11] Brudnyi, A. And Kinzebulatov, D.: Holomorphic almost periodic functions on coverings of complex manifolds. New York J. Math. 17A (2011), 267-300.

[12] Brudnyi, A. and Kinzebulatov, D.: On uniform subalgebras of $L^{\infty}$ on the unit circle generated by almost periodic functions. St. Petersburg J. Math. 19 (2008), 495-518.

[13] Brudnyi, A. And Kinzebulatov, D.: Towards Oka-Cartan theory for algebras of holomorphic functions on coverings of Stein manifolds II. To appear in Rev. Mat. Iberoam.

[14] Bungart, L.: On analytic fiber bundles. I. Holomorphic fiber bundles with infinite dimensional fibers. Topology 7 (1967), 55-68. 
[15] Bungart, L.: Holomorphic functions with values in locally convex spaces and applications to integral formulas. Trans. Amer. Math. Soc 111 (1964), 317-344; Errata, ibid. 113 (1964), 547.

[16] Favorov, S.: Zeros of holomorphic almost periodic functions. J. Anal. Math. 84 (2001), 51-66.

[17] Favorov, S.: Almost periodic divisors, holomorphic functions, and holomorphic mappings. Bull. Sci. Math. 127 (2003), 859-883.

[18] Favorov, S. And Rashkovskit, A.: Holomorphic almost periodic functions. Acta Appl. Math. 65 (2001), 217-235.

[19] Gamelin, T.: Uniform algebras. Second edition. Chelsea Publishing Co., New York, 1984.

[20] Grauert, H. and Remmert, R.: Theory of Stein spaces. Fundamental Principles of Mathematical Sciences 236, Springer-Verlag, Berlin-New York, 1979.

[21] Grigoryan, S.: Generalized analytic functions. Russian Math. Surveys 49 (1994), $1-40$.

[22] Gromov, M., Henkin G. And Shubin M.: Holomorphic $L^{2}$ functions on coverings of pseudoconvex manifolds. Geom. Funct. Anal. 8 (1998), 552-585.

[23] Gunning, R.: Introduction to holomorphic functions of several variables. III. Homological theory. The Wadsworth \& Brooks/Cole Mathematical Series, Wadsworth \& Brooks/Cole Advanced Books \& Software, Monterey, CA, 1990.

[24] Gunning, R. And Rossi, H.: Analytic functions of several complex variables. Prentice-Hall, 1965.

[25] Henkin, G. And Leiterer, J.: Theory of functions on complex manifolds. Monographs in Mathematics 79, Birkhäuser, 1984.

[26] Hirzebruch, F.: Topological methods in algebraic geometry. Third enlarged edition. Die Grundlehren der Mathematischen Wissenschaften 131, Springer-Verlag New York, New York, 1966.

[27] Jessen, B. and Tornehave, H.: Mean motions and zeros of almost periodic functions. Acta Math. 77 (1945), 137-279.

[28] Krantz, S.: Function theory of several complex variables. Second edition. AMS Chelsea Publishing, Providence, RI, 1992.

[29] LÁrusson, F.: Holomorphic functions of slow growth on nested covering spaces of compact manifolds. Canad. J. Math. 52 (2000), 982-998.

[30] Leiterer, J.: Banach coherent analytic Fréchet sheaves. Math. Nachr. 85 (1978), 91-109.

[31] Lempert, L. And Patyi, I.: Analytic sheaves in Banach spaces. Ann. Sci. École Norm. Sup. (4) 40 (2007), no. 3, 453-486.

[32] Levin, B.: Distribution of zeros of entire functions. Revised edition. Translations of Mathematical Monographs 5, American Mathematical Society, Providence, RI, 1980 .

[33] Lin, V.: Liouville coverings of complex spaces, and amenable groups. Math. USSRSb. 60 (1988), 197-216.

[34] Lyons, T. And Sullivan, D.: Function theory, random paths and covering spaces. J. Differential Geom. 19 (1984), 229-323.

[35] MaAk, W.: Eine neue Definition der fastperiodischen Funktionen. Abh. Math. Sem. Univ. Hamburg 11 (1936), 240-244. 
[36] Nagami, K.: Dimension theory. Pure and Applied Mathematics 37, Academic Press, New York-London 1970.

[37] von Neumann, J.: Almost periodic functions in a group. I.. Trans. Amer. Math. Soc. 36 (1934), 445-492.

[38] Ronkin, L.: Jessen's theorem for holomorphic almost periodic functions in tube domains. Sibirsk. Mat. Zh. 28 (1987), 199-204.

[39] Shubin, M.: Almost periodic functions and partial differential operators. Russian Math. Surveys 33 (1978), 1-52.

[40] Tornehave, H.: On the zeros of entire almost periodic function. Mat.-Fys. Medd. Danske Vid. Selsk. 42 (1989), 125-142.

[41] Vesentini, E.: Holomorphic almost periodic functions and positive-definite functions on Siegel domains. Ann. Mat. Pura Appl. (4) 102 (1973), 177-202.

[42] WEIL, A.: L'intégration dans les groupes topologiques et ses applications. Hermann, Paris, 1965.

Received September 24, 2013; revised November 12, 2013.

Alexander Brudnyi: Department of Mathematics and Statistics, University of Calgary, 2500 University Drive NW, Calgary, Alberta T2N 1N4, Canada.

E-mail: abrudnyi@ucalgary.ca

Damir Kinzebulatov: The Fields Institute, 222 College Street, Second Floor, Toronto, Ontario M5T 3J1, Canada.

E-mail: damir.kinzebulatov@utoronto.ca

Research of the first and the second authors was partially supported by NSERC. 